Supporting Information for

\title{
Reductive Cleavage of Tetrahydrofuryl Sulfur- Substituted Oxiranes: Application to the Formal Synthesis of Kumausyne and Kumausallene
}

Roberto Fernández de la Pradilla, * Carolina Alhambra, Alejandro Castellanos, Jorge Fernández, Pilar Manzano, Carlos Montero, Mercedes Ureña, and Alma Viso.

† Instituto de Química Orgánica, CSIC, Juan de la Cierva, 3, 28006 Madrid, Spain.

Fax: 34-91-564-4853

E-mail: iqofp19@iqog.csic.es 
Table of Contents

$\begin{array}{ll}\text { Materials and Methods. } & \text { S6 }\end{array}$

1.1. General Procedure for the Preparation of Silyl Ethers.

1.2. Synthesis of the tert-Butyldimethylsilyl ethers of $(2 R, 5 R)-5-$ Isopropyl-4-

(p-tolylsulfonyl)-2,5-dihydrofuran-2-methanol, (-)-8a, and (2S,5R)-5-Isopropyl-4-

(p-tolylsulfonyl)-2,5-dihydrofuran-2-methanol, (+)-9a.

1.3. Synthesis of the tert-Butyldimethylsilyl ethers of $(2 R, 5 R)-5-P h e n y l-4-$

(p-tolylsulfonyl)-2,5-dihydrofuran-2-methanol, (-)-8c, and (2S,5R)-5-Phenyl-4-

(p-tolylsulfonyl)-2,5-dihydrofuran-2-methanol, (+)-9c.

2.1. General Procedure for Nucleophilic Epoxidation of Vinyl Sulfoxides and Sulfones with $t \mathrm{BuOOK}$.

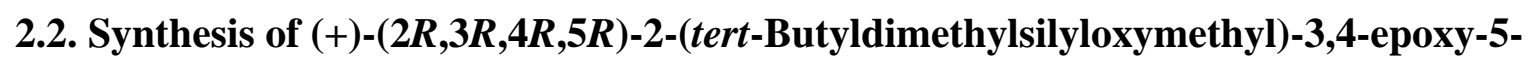
isopropyl-4-(p-tolylsulfonyl)tetrahydrofuran, $(+)-10 a$.

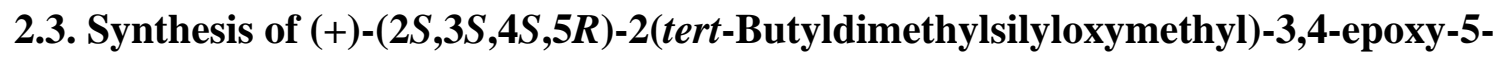
isopropyl-4-(p-tolylsulfonyl)tetrahydrofuran, $(+)-11$ a.

2.4. Synthesis of (-)-(2R,3R,4R,5R)-2-(tert-Butyldimethylsilyloxymethyl)-3,4-epoxy-5phenyl-4-(p-tolylsulfonyl)tetrahydrofuran, (-)-10c.

2.5. Synthesis of $(+)-(2 S, 3 S, 4 S, 5 R)-2-(t e r t-B u t y l d i m e t h y l s i l y l o x y m e t h y l)-3,4-e p o x y-5-$ phenyl-4-(p-tolylsulfonyl)tetrahydrofuran, $(+)-11 c$.

3.1. General Procedure for Deprotection of Silyl Ethers with $n B u_{4} N F / A c O H$.

3.2. Synthesis of $(+)-(2 S, 3 S, 4 S, 5 R)-3,4-E p o x y-5-i s o p r o p y l-4-$

(p-tolylsulfonyl)tetrahydrofuran-2-methanol, $(+)-4 a$.

3.3. Synthesis of $(-)-(2 R, 3 R, 4 S, 5 R)-3,4-E$ poxy-5-phenyl-4-

(p-tolylsulfonyl)tetrahydrofuran-2-methanol, (-)-5c.

3.4. Synthesis of $(+)-(2 S, 3 S, 4 R, 5 R)-3,4-E p o x y-5-p h e n y l-4-$

(p-tolylsulfonyl)tetrahydrofuran-2-methanol, $(+)-4 c$.

4.1. General Procedure for Reductive Cleavage of Sulfinyl and Sulfonyl

Oxiranes with (PhSe) $)_{2}$ and $\mathrm{NaBH}_{4}$.

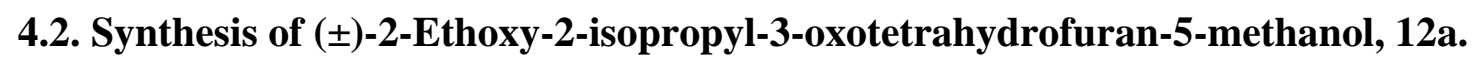

4.3. Synthesis of $( \pm)$-2-Ethoxy-4-phenylselenyl-2-isopropyl-3-oxotetrahydrofuran-5- 
methanol, 13a.

4.4. Synthesis of $( \pm)$-2-Isopropyl-3-oxotetrahydrofuran-5-methanol, 14a and 15a.

4.5. Synthesis of $( \pm)-2-E t h y l-3-0 x o t e t r a h y d r o f u r a n-5-m e t h a n o l, 14 b$ and $15 b$.

4.6. Synthesis of ( \pm )-2-phenyl-3-oxotetrahydrofuran-5-methanol, 14c and 15c.

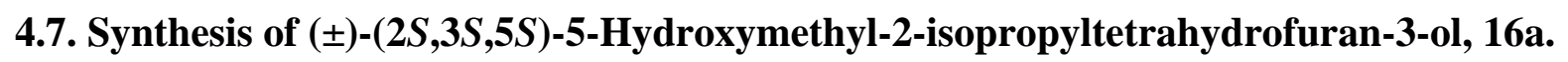

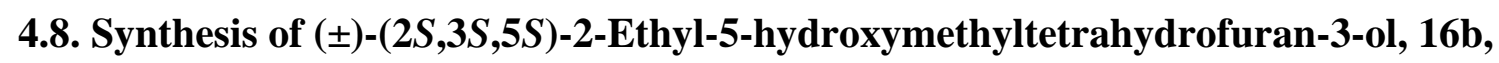

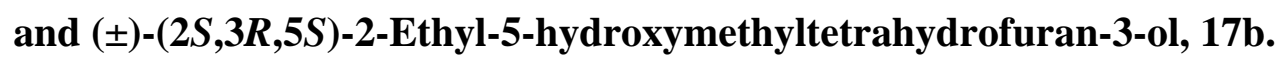

4.9. Synthesis of $( \pm)-(2 S, 3 S, 5 S)-2-P h e n y l-5-h y d r o x y m e t h y l t e t r a h y d r o f u r a n-3-o l, 16 c$,

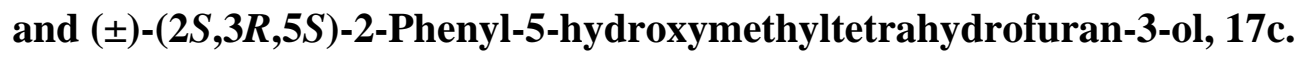

4.10. Synthesis of $( \pm)-(2 S, 3 S, 5 S)-2-(p$-Fluorophenyl)-5-hydroxymethyltetrahydrofuran3-ol, 16d, and ( \pm )-(2S,3R,5S)-2-(p-Fluorophenyl)-5-hydroxymethyltetrahydrofuran-3-ol, 17d.

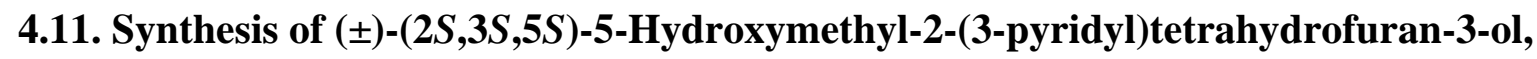
$16 \mathrm{e}$, and $( \pm)-(2 S, 3 R, 5 S)-5-\mathrm{Hydroxymethyl-2-(3-pyridyl)tetrahydrofuran-3-ol,} 16 \mathrm{e}$.

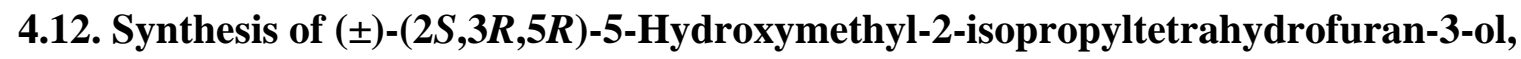

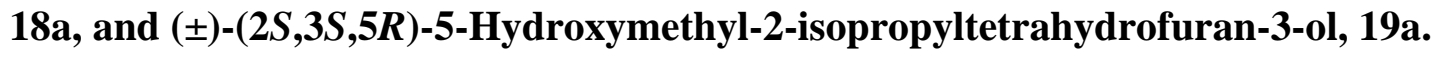

4.13. Synthesis of $( \pm)-(2 S, 3 R, 5 R)-2-(p$-Fluorophenyl)-5-hydroxymethyltetrahydrofuran3-ol, 18d, and ( \pm )-(2S,3S,5R)-2-(p-Fluorophenyl)-5-hydroxymethyltetrahydrofuran-3-ol, 19d.

5.1. General Procedure for Cleavage of Sulfonyl Oxiranes with $\mathrm{MgBr}_{2}$ and $\mathrm{MgI}_{2}$.

5.2. Synthesis of $( \pm)-(2 S, 4 R, 5 S)-4-B r o m o-5-(h y d r o x y m e t h y l)-2-p h e n y l-3-$ oxotetrahydrofuran, 20.

5.3. Synthesis of $(2 R, 5 S)$-5-(hydroxymethyl)-2-isopropyl-3-oxotetrahydrofuran, 15a.

5.4. Synthesis of $(+)-(2 R, 5 S)-5-(t e r t-B u t y l d i m e t h y l s i l y l o x y m e t h y l)-2$

-isopropyl-3-oxotetrahydrofuran, $(+)-21 a$.

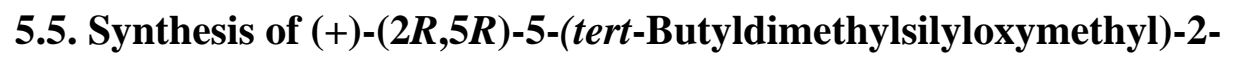

isopropyl-3-oxotetrahydrofuran, $(+)-22 a$.

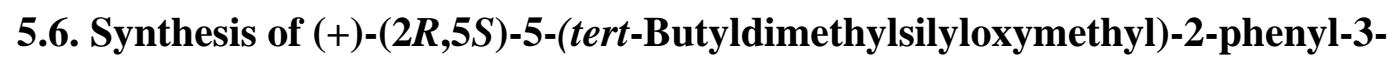
oxotetrahydrofuran, $(+)-21 c$.

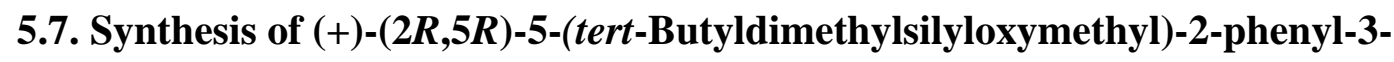
oxotetrahydrofuran, $(+)-22 c$.

6.1. Synthesis of (-)-(2R,3R,5S)-5-(tert-Butyldimethylsilyloxymethyl)-2-phenyl- 
tetrahydrofuran-3-ol, (-)-23c.

6.2. Synthesis of (-)-(2R,3R,5R)-5-(tert-Butyldimethylsilyloxymethyl)-2-phenyl-

tetrahydrofuran-3-ol, (-)-24c.

S25

7.1. Synthesis of the tert-Butyldimethylsilyl ether of $(+)-\left(2 R, 3 R, 4 R, 5 S, S_{S}\right)-3,4-$

Epoxy-4-(p-tolylsulfinyl)-5-vinyl-tetrahydrofuran-2-methanol, (+)-28a.

S26

7.2. Synthesis of the tert-Butyldiphenylsilyl ether of $(+)-\left(2 R, 3 R, 4 R, 5 S, S_{S}\right)-3,4-$

Epoxy-4-(p-tolylsulfinyl)-5-vinyl-tetrahydrofuran-2-methanol, (+)-28b.

S26

7.3. Synthesis of the tert-Butyldiphenylsilyl ether of $(+)-(2 R, 3 R, 4 R, 5 S)-3,4-$

Epoxy-4-(p-tolylsulfonyl)-5-vinyl-tetrahydrofuran-2-methanol, (-)-29b.

S27

7.4. General Procedure for Benzylation of Sulfinyl and Sulfonyl

Tetrahydrofuran Methanols.

7.5. Synthesis of the benzyl ethers of $(+)-\left(2 R, 3 R, 4 R, 5 S, S_{S}\right)-3,4-E p o x y-4-$

(p-tolylsulfinyl)-5-vinyl-tetrahydrofuran-2-methanol, 28c, and of

(+)-(2R,3R,4R,5S)-3,4-Epoxy-4-(p-tolylsulfonyl)-5-

vinyl-tetrahydrofuran-2-methanol, 29c.

S28

8.1. Synthesis of the tert-Butyldiphenylsilyl ether of $(Z, 2 S)$-5-ethylidene-4oxotetrahydrofuran-2-methanol, (-)-31b.

8.2. Synthesis of (+)-(2S,4S,5S)-2-(tert-Butyldiphenylsilyloxymethyl)-5-

vinyl-tetrahydrofuran-4-ol, (+)-26b, and (-)-(2S,4R,5S)-2-

(tert-Butyldiphenylsilyloxymethyl)-5-vinyl-tetrahydrofuran-4-ol, (-)-32b.

8.3. Synthesis of $(2 S, 4 S, 5 S)-4-H y d r o x y-5$-vinyl-tetrahydrofuran-2-methanol, $(+)-26 \mathrm{~d}$,

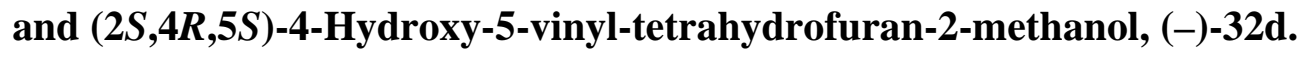

8.4. Synthesis of (+)-(2S,4S,5S)-2-(Benzyloxymethyl)-5-vinyl-tetrahydrofuran-4-ol,

(+)-26c, and (2S,4R,5S)-2-(Benzyloxymethyl)-5-vinyl-tetrahydrofuran-4-ol, 32c.

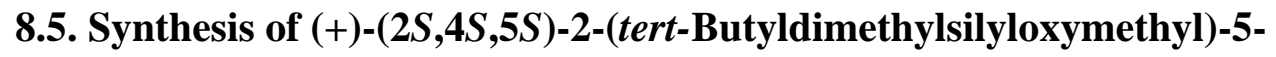

vinyl-tetrahydrofuran-4-ol, (+)-26a, and (-)-(2S,4R,5S)-2-

(tert-Butyldimethylsilyloxymethyl)-5-vinyl-tetrahydrofuran-4-ol, (-)-32a.

S33

8.6. Desilylation of $(+)-26 a$.

9.1. General Procedure for the Oxidation of Hydroxy Vinyl Tetrahydrofurans to Dioxabicyclic Lactols.

9.2. Synthesis of ent-Kumausyne precursor, (-)-(4S,6S,8R)-6- 
(tert-Butyldiphenylsilyloxymethyl)-1,5-dioxabicyclo[3.3.0 $\left.{ }^{4,8}\right]$ octan-2-ol, (-)-25b. S34

9.3. Wacker oxidation of diol (+)-26d. Synthesis of $(-)-(4 S, 6 S, 8 R)-6-$

(hydroxymethyl)-1,5-dioxabicyclo[3.3.0 $\left.{ }^{4,8}\right]$ octan-2-ol, (-)-25d.

10.1. Wittig reaction on lactol (-)-25d. Synthesis of $(+)-(2 S, 4 S, 5 S)-2-$

Hydroxymethyl-5-(pent-2-en-4-ynyl)-tetrahydrofuran-4-ol. (+)-34.

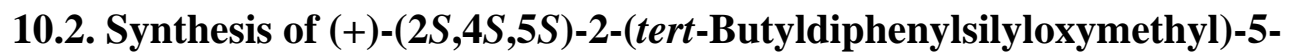

[1-carbomethoxy-1-(E)-propen-3-yl]tetrahydrofuran-4-ol, (+)-35b.

10.3. Synthesis of $(2 S, 4 S, 5 S)-5-$

[1-carbomethoxy-1-(E)-propen-3-yl]-2-hydroxymethyltetrahydrofuran-4-ol, 35d.

11.1. Synthesis of ent-Kumausallene precursor, $(-)-(2 R, 4 S, 6 S, 8 R)-2-$

Carbomethoxymethyl-1,5-dioxabicyclo[3.3.0 $\left.{ }^{4,8}\right]$ octan-6-methanol, (-)-27. 


\section{Materials and Methods}

Reagents and solvents were handled by using standard syringe techniques. All reactions were carried out under an argon atmosphere. Hexane, toluene and $\mathrm{CH}_{2} \mathrm{Cl}_{2}$ were distilled from $\mathrm{CaH}_{2}$, and THF and $\mathrm{Et}_{2} \mathrm{O}$ from sodium. $(\mathrm{MeO})_{2} \mathrm{P}(\mathrm{O}) \mathrm{Me}, \mathrm{Et}_{3} \mathrm{~N}$, and $i-\mathrm{Pr}_{2} \mathrm{NH}$ were distilled from $\mathrm{CaH}_{2}$. Crude products were purified by flash chromatography on 230-400 mesh silica gel with distilled solvents. Analytical TLC was carried out on silica gel plates with detection by UV light, iodine, acidic vanillin solution, 10\% phosphomolybdic acid solution in ethanol. All reagents were commercial products. Organolithium

reagents were titrated prior to use. ${ }^{1} \mathrm{NaH}$ and $\mathrm{KH}(60 \%$ in mineral oil) were washed repeatedly with dry hexane and dried prior to use. Through this section, the volume of solvents is reported in $\mathrm{mL} / \mathrm{mmol}$ of starting material. ${ }^{1} \mathrm{H}$ and ${ }^{13} \mathrm{C}$ NMR spectra were recorded at 200,300 or $400 \mathrm{MHz}\left({ }^{1} \mathrm{H}\right)$ using $\mathrm{CDCl}_{3}$ as solvent and with the residual solvent signal as internal reference $\left(\mathrm{CDCl}_{3}, 7.24\right.$ and $\left.77.0 \mathrm{ppm}\right)$. The following abbreviations are used to describe peak patterns when appropriate: $s$ (singlet), $d$ (doublet), $t$ (triplet), q (quartet), m (multiplet), br (broad), ap (apparent). Melting points are uncorrected. Optical rotations were measured at $20{ }^{\circ} \mathrm{C}$ using a sodium lamp and in $\mathrm{CHCl}_{3}$ solution. Low resolution mass spectra were recorded using the electronic impact technique with an ionization energy of $70 \mathrm{eV}$ or using the atmospheric pressure chemical ionization (APCI) or electrospray (ES) chemical ionization techniques in its positive or negative modes.

\subsection{General Procedure for the Preparation of Silyl Ethers.}

To a solution of the substrate in anhydrous $\mathrm{CH}_{2} \mathrm{Cl}_{2}(0.4 \mathrm{~mL} / \mathrm{mmol})$ at $\mathrm{rt}$ was added imidazole $(1.5$ equiv) and DMAP (0.05 equiv). The solution was cooled to $0{ }^{\circ} \mathrm{C}$ and TBDMSCl or TBDPSCl (1.5 equiv) was added. The mixture was allowed to warm up to rt and monitored by TLC. Upon completion the reaction was quenched with $\mathrm{H}_{2} \mathrm{O}(5 \mathrm{~mL} / \mathrm{mmol})$ and saturated $\mathrm{NH}_{4} \mathrm{Cl}$ solution $(5 \mathrm{~mL} / \mathrm{mmol})$. The layers were separated, the aqueous phase was extracted with $\mathrm{CH}_{2} \mathrm{Cl}_{2}(3 \times 5 \mathrm{~mL} / \mathrm{mmol})$ and the combined organic extracts were washed with a saturated solution of $\mathrm{NaCl}(5 \mathrm{~mL} / \mathrm{mmol})$, dried over $\mathrm{MgSO}_{4}$, filtered and concentrated under reduced pressure to give a crude product that was purified by column chromatography on silica gel using a gradient of the appropriate solvents. 
1.2. Synthesis of the tert-Butyldimethylsilyl ethers of $(2 R, 5 R)-5-I s o p r o p y l-4-(p-t o l y l s u l f o n y l)-2,5-$ dihydrofuran-2-methanol, (-)-8a, and (2S,5R)-5-Isopropyl-4-(p-tolylsulfonyl)-2,5-dihydrofuran-2methanol, (+)-9a.
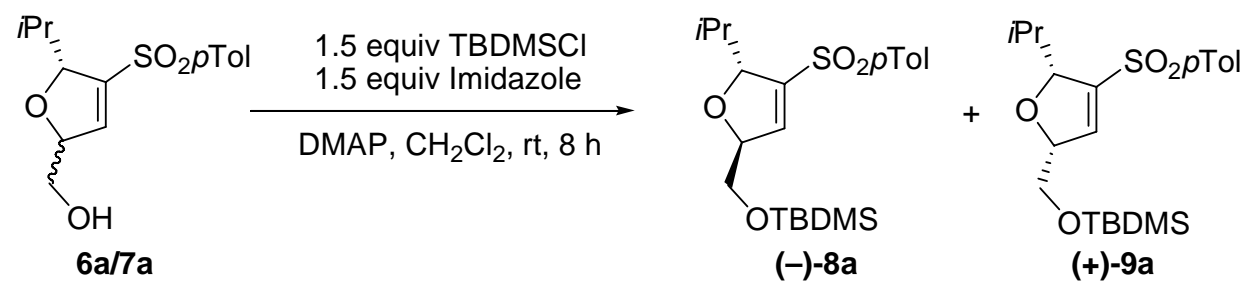

From a mixture of sulfonyl dihydrofurans $6 \mathbf{6 a}$ and $7 \mathbf{a}^{2}$ (189 $\mathrm{mg}, 0.64 \mathrm{mmol}, 1.0$ equiv), with TBDMSCl (152 mg, $0.96 \mathrm{mmol}, 1.5$ equiv) according to the general procedure $(8 \mathrm{~h}) \mathrm{a} 43: 57$ mixture of silyl ethers (-)-8a and (+)-9a was obtained. Purification by chromatography $\left(30-70 \% \mathrm{CH}_{2} \mathrm{Cl}_{2}\right.$-hexane) gave (-)-8a (76 mg, 29\%) and (+)-9a (137 mg, 61\%) as colorless oils.

Data for (-)-8a: $\boldsymbol{R}_{\boldsymbol{f}}=0.25\left(\mathrm{CH}_{2} \mathrm{Cl}_{2}\right) .[\alpha]^{20}{ }_{\mathbf{D}}=-105.1(c=1.39) .{ }^{1} \mathbf{H} \mathbf{~ N M R}(\mathbf{3 0 0} \mathbf{~ M H z}) \delta-0.04(\mathrm{~s}, 3$ H), $-0.01(\mathrm{~s}, 3 \mathrm{H}), 0.69(\mathrm{~d}, 3 \mathrm{H}, J=6.8 \mathrm{~Hz}), 0.81$ (s, $9 \mathrm{H}), 0.98(\mathrm{~d}, 3 \mathrm{H}, J=6.9 \mathrm{~Hz}), 2.06$ (hept d, $1 \mathrm{H}, J$ $=6.9,1.5 \mathrm{~Hz}), 2.43(\mathrm{~s}, 3 \mathrm{H}), 3.56(\mathrm{dd}, 1 \mathrm{H}, J=10.4,5.6 \mathrm{~Hz}), 3.71(\mathrm{dd}, 1 \mathrm{H}, J=10.4,3.8 \mathrm{~Hz}), 4.80$ (ap tt, $1 \mathrm{H}, J=6.8,6.1,1.6 \mathrm{~Hz}), 4.84(\mathrm{dd}, 1 \mathrm{H}, J=6.1,1.4 \mathrm{~Hz}), 6.72($ ap t, $1 \mathrm{H}, J=1.5 \mathrm{~Hz}), 7.32(\mathrm{~d}, 2 \mathrm{H}, J=$ $8.5 \mathrm{~Hz}), 7.76(\mathrm{~d}, 2 \mathrm{H}, J=8.6 \mathrm{~Hz}) .{ }^{13} \mathrm{C}$ NMR (75 MHz) $\delta-5.5,-5.5,14.3,18.2,19.9,21.6,25.8$ (3 C), 31.8, 64.9, 86.1, 89.4, 128.1 (2 C), 129.9 (2 C), 136.7, 140.6, 144.1, 144.9. IR (film): 3355, 2956, 2929, $2855,1597,1463,1322,1158,836,666 \mathrm{~cm}^{-1}$. MS (ES): $843[2 \mathrm{M}+\mathrm{Na}]^{+}, 433[\mathrm{M}+\mathrm{Na}]^{+}(100 \%), 411$ $[\mathrm{M}+1]^{+}$. Anal. calcd for $\mathrm{C}_{21} \mathrm{H}_{34} \mathrm{O}_{4} \mathrm{SSi}$ : C, 61.42; H, 8.35; S, 7.81. Found: C, 61.75; H, 8.04; S, 7.60.

Data for (+)-9a: $\quad \boldsymbol{R}_{\boldsymbol{f}}=0.16\left(\mathrm{CH}_{2} \mathrm{Cl}_{2}\right) . \quad[\alpha]^{\mathbf{2 0}}{ }_{\mathbf{D}}=+63.0(c=1.55) .{ }^{\mathbf{1}} \mathbf{H} \mathbf{N M R}(\mathbf{3 0 0} \mathbf{M H z}) \delta 0.02(\mathrm{~s}, 3$ H), $0.03(\mathrm{~s}, 3 \mathrm{H}), 0.63(\mathrm{~d}, 3 \mathrm{H}, J=6.7 \mathrm{~Hz}), 0.85$ (s, $9 \mathrm{H}), 0.96$ (d, $3 \mathrm{H}, J=7.0 \mathrm{~Hz}), 2.08$ (hept d, 1H, $J=$ 6.9, $1.4 \mathrm{~Hz}), 2.43$ (s, $3 \mathrm{H}), 3.55$ (dd, $1 \mathrm{H}, J=10.0,6.9 \mathrm{~Hz}), 3.76$ (dd, $1 \mathrm{H}, J=10.1,5.2 \mathrm{~Hz}), 4.78-4.83$ $(\mathrm{m}, 2 \mathrm{H}), 6.74(\mathrm{~s}, 1 \mathrm{H}), 7.33(\mathrm{~d}, 2 \mathrm{H}, J=9.0 \mathrm{~Hz}), 7.76(\mathrm{~d}, 2 \mathrm{H}, J=8.3 \mathrm{~Hz}) .{ }^{13} \mathbf{C} \mathbf{N M R}(75 \mathrm{MHz}) \delta$ -5.4 (2 C), 14.9, 18.3, 19.8, 21.7, 25.8 (3 C), 30.4, 64.9, 85.1, 89.1, 128.1 (2 C), 129.9 (2 C), 136.6, 140.9, 144.2, 144.9. IR (film): 3500, 2956, 2932, 2876, 1786, 1757, 1597, 1558, 1464, 1323, 1154, 667 $\mathrm{cm}^{-1}$. MS (ES): $843[2 \mathrm{M}+\mathrm{Na}]^{+}, 433[\mathrm{M}+\mathrm{Na}]^{+}, 411[\mathrm{M}+1]^{+}(100 \%)$. Anal. calcd for $\mathrm{C}_{21} \mathrm{H}_{34} \mathrm{O}_{4} \mathrm{SSi}: \mathrm{C}$, 61.42; H, 8.35; S, 7.81. Found: C, 61.57; H, 8.42; S, 7.89. 
1.3. Synthesis of the tert-Butyldimethylsilyl ethers of $(2 R, 5 R)-5-P h e n y l-4-(p$-tolylsulfonyl)-2,5dihydrofuran-2-methanol, (-)-8c, and (2S,5R)-5-Phenyl-4-(p-tolylsulfonyl)-2,5-dihydrofuran-2methanol, (+)-9c.

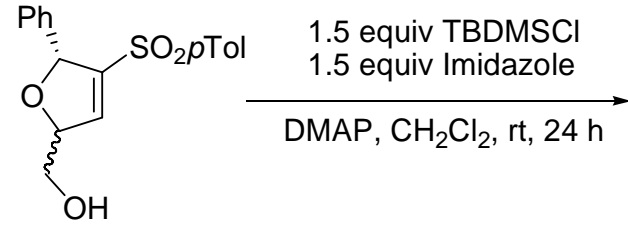

$6 c / 7 c$

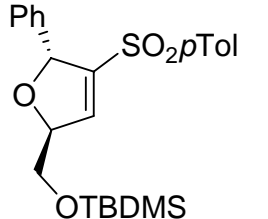

$(-)-8 c$

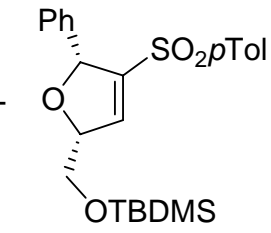

$(+)-9 c$

From a mixture of sulfonyl dihydrofurans $6 \mathbf{c}$ and $7 \mathbf{c}^{2}(93 \mathrm{mg}, 0.280 \mathrm{mmol}, 1.0$ equiv), with TBDMSCl (66.5 $\mathrm{mg}, 0.420 \mathrm{mmol}, 1.5$ equiv) according to the general procedure (24 h) a 36:64 mixture of silyl ethers (-)-8c and (+)-9c was obtained. Purification by chromatography $\left(20-50 \% \mathrm{CH}_{2} \mathrm{Cl}_{2}\right.$-hexane) gave (-)-8c (42 mg, 34\%), (+)-9c (51 mg, 41\%) as white solids and $14 \mathrm{mg}(11 \%)$ of a mixture of (-)-8c and $(+)-9 c$.

Data for (-)-8c: $\mathbf{m p}: 80-81{ }^{\circ} \mathrm{C} . \quad \boldsymbol{R}_{\boldsymbol{f}}=0.15\left(80 \% \mathrm{CH}_{2} \mathrm{Cl}_{2}\right.$-hexane). $[\boldsymbol{\alpha}]^{\mathbf{2 0}}{ }_{\mathbf{D}}=-129.8(c=1.90) .{ }^{\mathbf{1}} \mathbf{H}$ NMR (300 MHz) $\delta 0.05$ (s, $6 \mathrm{H}), 0.88$ (s, $9 \mathrm{H}), 2.31$ (s, $3 \mathrm{H}), 3.73(\mathrm{dd}, 1 \mathrm{H}, J=10.4,5.8 \mathrm{~Hz}), 3.82$ (dd, $1 \mathrm{H}, J=10.4,4.2 \mathrm{~Hz}), 5.13$ (q, $1 \mathrm{H}, J=5.7 \mathrm{~Hz}), 5.87$ (dd, $1 \mathrm{H}, J=5.8,1.9 \mathrm{~Hz}), 6.97-7.29$ (m, 10 H). ${ }^{13}$ C NMR (75 MHz) $\delta-5.5,-5.4,18.3,21.5,25.8$ (3 C), 64.9, 86.3, 86.9, 127.6 (2 C), 127.9 (2 C), 128.2 (2 C), 128.5 (C), 129.3 (2 C), 136.3, 137.9, 140.4, 144.1, 145.7. IR (KBr): 3435, 2928, 2855, $1631,1595,1454,1320,1252,1155,593 \mathrm{~cm}^{-1}$. MS (ES): $911[2 \mathrm{M}+\mathrm{Na}]^{+}(100 \%), 467[\mathrm{M}+\mathrm{Na}]^{+}, 462$ $\left[\mathrm{M}+\mathrm{H}_{2} \mathrm{O}\right]^{+}, 445[\mathrm{M}+1]^{+}$. Anal. calcd for $\mathrm{C}_{24} \mathrm{H}_{32} \mathrm{O}_{4} \mathrm{SSi}$ : C, 64.83; H, 7.25; S, 7.21. Found: C, 65.01; $\mathrm{H}$, 7.00; S, 6.97.

Data for (+)-9c: $\mathbf{m p :} 98-99{ }^{\circ} \mathrm{C} . \quad \boldsymbol{R}_{\boldsymbol{f}}=0.05\left(80 \% \mathrm{CH}_{2} \mathrm{Cl}_{2}\right.$-hexane). $[\boldsymbol{\alpha}]^{\mathbf{2 0}}{ }_{\mathbf{D}}=+29.2(c=2.57) .{ }^{\mathbf{1}} \mathbf{H}$ NMR (300 MHz) $\delta 0.04$ (s, 6 H), 0.87 (s, 9 H), 2.29 (s, 3 H), 3.81 (dd, 1 H, J = 10.8, 4.5 Hz), 3.89 $(\mathrm{dd}, 1 \mathrm{H}, J=10.8,3.7 \mathrm{~Hz}), 5.02$ (q, $1 \mathrm{H}, J=4.8 \mathrm{~Hz}), 5.86(\mathrm{dd}, 1 \mathrm{H}, J=4.8,2.2 \mathrm{~Hz}), 6.91-7.24$ (m, 10 H). ${ }^{13}$ C NMR (75 MHz) $\delta-5.6,-5.5,18.3,21.5,25.9$ (3 C), 64.8, 85.9, 86.4, 127.8 (2 C), 127.9 (2 C), 128.3 (2 C), 128.5, 129.2 (2 C), 136.3, 137.6, 140.7, 143.9, 145.5. IR (KBr): 3435, 3065, 2929, 2856, 1626, 1458, 1321, 1261,1157, 1133, 1090, 925, 831,811, 777, 758, 700,668, $592 \mathrm{~cm}^{-1}$. MS (ES): $911[2 \mathrm{M}+\mathrm{Na}]^{+}, 467[\mathrm{M}+\mathrm{Na}]^{+} \quad(100 \%), 462\left[\mathrm{M}+\mathrm{H}_{2} \mathrm{O}\right]^{+}, 445[\mathrm{M}+1]^{+}$. Anal. calcd for $\mathrm{C}_{24} \mathrm{H}_{32} \mathrm{O}_{4} \mathrm{SSi}: \mathrm{C}, 64.83$; H, 7.25; S, 7.21. Found: C, 64.65; H, 7.40; S, 6.98.

\subsection{General Procedure for Nucleophilic Epoxidation of Vinyl Sulfoxides and Sulfones with tBuOOK.}

A two-necked round-bottomed flask fitted with a tube in $\mathrm{T}$ for entrance and exit of argon and a polyethylene stopper, was charged with anhydrous THF $(5 \mathrm{~mL} / \mathrm{mmol})$ and 2-4 equiv of oil free $\mathrm{KH}$ 
(washed with hexane and dried), the mixture was cooled to $0{ }^{\circ} \mathrm{C}$ and then $2-4.5$ equiv of $t \mathrm{BuOOH}(80 \%$ in $t \mathrm{BuOO} t \mathrm{Bu}$ ) was added. After stirring at $\mathrm{rt}$ for $10 \mathrm{~min}$, the resulting solution was cooled to $0{ }^{\circ} \mathrm{C}$ and a solution of 1 equiv of the corresponding vinyl sulfoxide in THF ( $7 \mathrm{~mL} / \mathrm{mmol})$, previously dried over 4 $\AA$ sieves, was added dropwise. The reaction mixture was stirred at $0{ }^{\circ} \mathrm{C}$ until starting material disappearance, monitored by TLC. The reaction was then quenched with a $10 \%$ solution of $\mathrm{Na}_{2} \mathrm{~S}_{2} \mathrm{O}_{4}(4$ $\mathrm{mL} / \mathrm{mmol})$, diluted whith EtOAc $(10 \mathrm{~mL} / \mathrm{mmol})$ and the layers were separated. The aqueous layer was extracted with EtOAc ( 3 times, $10 \mathrm{~mL} / \mathrm{mmol}$ ) and the combined organic extracts were washed with a saturated solution of $\mathrm{NaCl}(4 \mathrm{~mL} / \mathrm{mmol})$, dried over anhydrous $\mathrm{MgSO}_{4}$, filtered and concentrated under reduced pressure to give a crude product, which was purified by column chromatography on silica gel, using a gradient of mixtures of EtOAc-hexane or EtOAc- $\mathrm{CH}_{2} \mathrm{Cl}_{2}$. Product ratios were determined by integration of well resolved signals in the ${ }^{1} \mathrm{H}$ NMR of the crude reaction mixtures.

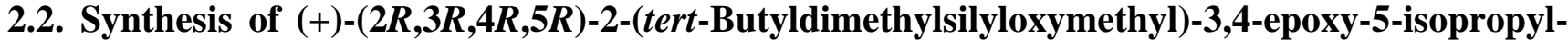
4-(p-tolylsulfonyl)tetrahydrofuran, $(+)-10 a$.

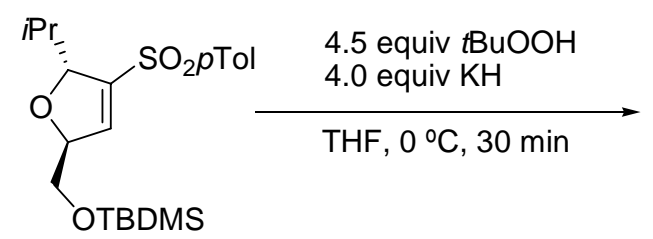

$(-)-8 a$

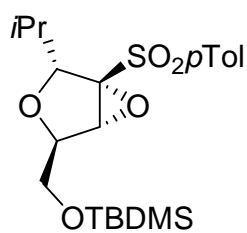

$(+)-10 a$

From a solution of sulfonyl dihydrofuran (-)-8a $(38.4 \mathrm{mg}, 0.094 \mathrm{mmol}, 1.0$ equiv) in $0.7 \mathrm{~mL}$ of THF, with $t$ BuOOK (4.0 equiv, in THF), according to the general procedure $\left(0^{\circ} \mathrm{C}, 30 \mathrm{~min}\right)$, sulfonyl oxirane (+)-10a was obtained as a single isomer. Purification by chromatography $(2-10 \%$ EtOAc-hexane) gave (+)-10a $(28.5 \mathrm{mg}, 71 \%)$ as a colorless oil.

Data for (+)-10a: $\boldsymbol{R}_{\boldsymbol{f}}=0.38\left(100 \% \mathrm{CH}_{2} \mathrm{Cl}_{2}\right) .[\alpha]^{\mathbf{2 0}}{ }_{\mathbf{D}}=+12.0(c=0.65) .{ }^{\mathbf{1}} \mathbf{H} \mathbf{N M R}(\mathbf{3 0 0} \mathbf{~ M H z}) \delta 0.07$ $(\mathrm{s}, 3 \mathrm{H}), 0.08(\mathrm{~s}, 3 \mathrm{H}), 0.63(\mathrm{~d}, 3 \mathrm{H}, J=7.0 \mathrm{~Hz}), 0.90(\mathrm{~s}, 9 \mathrm{H}), 0.96(\mathrm{~d}, 3 \mathrm{H}, J=7.0 \mathrm{~Hz}), 1.87$ (ap hept d, $1 \mathrm{H}, J=6.9,2.9 \mathrm{~Hz}), 2.45(\mathrm{~s}, 3 \mathrm{H}), 3.69-3.73(\mathrm{~m}, 2 \mathrm{H}), 3.84(\mathrm{~s}, 1 \mathrm{H}), 3.95$ (ap t, $1 \mathrm{H}, J=5.1,4.5 \mathrm{~Hz})$, $4.52(\mathrm{~d}, 1 \mathrm{H}, J=2.9 \mathrm{~Hz}), 7.35(\mathrm{~d}, 2 \mathrm{H}, J=8.4 \mathrm{~Hz}), 7.81(\mathrm{~d}, 2 \mathrm{H}, J=8.3 \mathrm{~Hz}) .{ }^{13} \mathbf{C} \mathbf{N M R}(75 \mathbf{M H z}) \delta$ -5.5 (2 C), 15.9, 18.3, 20.3, 21.7, 25.9 (3 C), 29.5, 63.0, 64.7, 76.3, 77.2, 78.9, 129.2 (2 C), 129.8 (2 C), 134.0, 144.9. IR (film): 3065, 2956, 2930, 2855, 1597, 1471, 1331, 1159, 1256, 838, $668 \mathrm{~cm}^{-1}$. MS (ES): $449[\mathrm{M}+\mathrm{Na}]^{+}$. Anal. calcd for $\mathrm{C}_{21} \mathrm{H}_{34} \mathrm{O}_{5} \mathrm{SSi}$ : C, 59.12; H, 8.03; S, 7.52. Found: C, 58.94; H, 8.17; S, 7.38. 
2.3. Synthesis of $(+)-(2 S, 3 S, 4 S, 5 R)$-2(tert-Butyldimethylsilyloxymethyl)-3,4-epoxy-5-isopropyl-4(p-tolylsulfonyl)tetrahydrofuran, $(+)-11 a$.

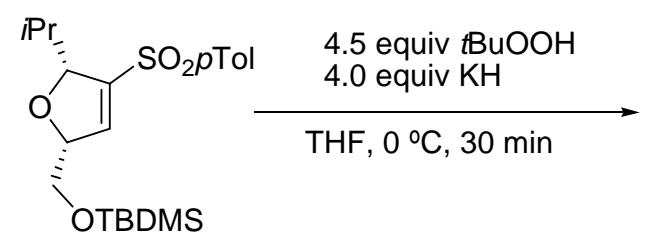

$(+)-9 a$

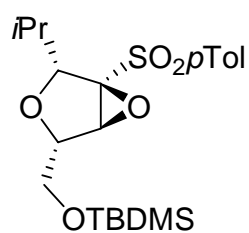

$(+)-11 a$

From a solution of sulfonyl dihydrofuran (+)-9a $(62 \mathrm{mg}, 0.151 \mathrm{mmol}, 1.0$ equiv) in $1.1 \mathrm{~mL}$ of THF, with $t \mathrm{BuOOK}$ (4.0 equiv, in THF), according to the general procedure $\left(0^{\circ} \mathrm{C}, 30 \mathrm{~min}\right)$, sulfonyl oxirane (+)-11a was obtained as a single isomer. Purification by chromatography (2-10\% EtOAc-hexane) gave (+)-11a (52 mg, 81\%) as a colorless oil.

Data for (+)-11a: $\boldsymbol{R}_{\boldsymbol{f}}=0.32\left(100 \% \mathrm{CH}_{2} \mathrm{Cl}_{2}\right) .[\alpha]^{\mathbf{2 0}}{ }_{\mathbf{D}}=+14.5(c=2.57) .{ }^{1} \mathbf{H}$ NMR (300 MHz) $\delta 0.05$ $(\mathrm{s}, 3 \mathrm{H}), 0.06(\mathrm{~s}, 3 \mathrm{H}), 0.87(\mathrm{~s}, 9 \mathrm{H}), 1.01(\mathrm{t}, 6 \mathrm{H}, J=6.9 \mathrm{~Hz}), 2.37$ (ap hept d, $1 \mathrm{H}, J=6.7,3.2 \mathrm{~Hz}), 2.45$ (s, $3 \mathrm{H}), 3.61-3.73(\mathrm{~m}, 2 \mathrm{H}), 3.96(\mathrm{ap} \mathrm{t}, 1 \mathrm{H}, J=5.1,3.8 \mathrm{~Hz}), 4.01$ (d, $1 \mathrm{H}, J=3.4 \mathrm{~Hz}), 4.02$ (s, $1 \mathrm{H})$, $7.35(\mathrm{~d}, 2 \mathrm{H}, J=8.0 \mathrm{~Hz}), 7.80(\mathrm{~d}, 2 \mathrm{H}, J=8.3 \mathrm{~Hz}) .{ }^{13} \mathbf{C}$ NMR (75 MHz) $\delta-5.5,-5.4,17.8,18.2,21.3$, 21.7, 25.8 (3 C), 29.5, 62.4, 66.9, 77.7, 78.0, 84.5, 129.3 (2 C), 129.8 (2 C), 133.6, 145.9. IR (film): 3384, 2956, 2930, 2876, 2855, 1597, 1471, 1332, 1257, 1150, 1086, $838 \mathrm{~cm}^{-1}$. MS (ES): $427[\mathrm{M}+1]^{+}$, $449[\mathrm{M}+\mathrm{Na}]^{+}(100 \%)$. Anal. calcd for $\mathrm{C}_{21} \mathrm{H}_{34} \mathrm{O}_{5} \mathrm{SSi}: \mathrm{C}, 59.12 ; \mathrm{H}, 8.03 ; \mathrm{S}, 7.52$. Found: C, 59.36; $\mathrm{H}$, $8.29 ; \mathrm{S}, 7.73$.

2.4. Synthesis of (-)-(2R,3R,4R,5R)-2-(tert-Butyldimethylsilyloxymethyl)-3,4-epoxy-5-phenyl-4(p-tolylsulfonyl)tetrahydrofuran, (-)-10c.

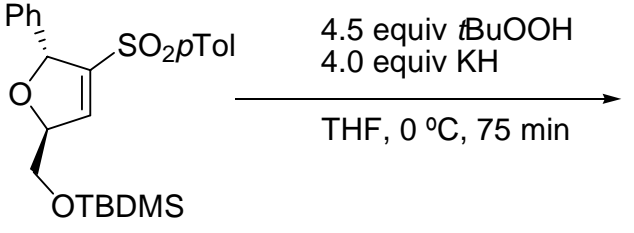

$(-)-8 c$

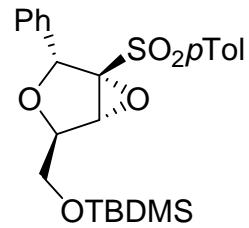

$(-)-10 c$

From a solution of sulfonyl dihydrofuran (-)-8c (77 mg, $0.173 \mathrm{mmol}, 1.0$ equiv) in $1.5 \mathrm{~mL}$ of THF, with $t$ BuOOK (4 equiv, in THF) according to the general procedure $\left(0^{\circ} \mathrm{C}, 75 \mathrm{~min}\right)$, sulfonyl oxirane (-)10c was obtained as a single isomer. Purification by chromatography (2-10\% EtOAc-hexane) gave (-)10c (66 mg, 83\%) as a colorless oil and unreacted (-)-8c (7 mg, 7\%).

Data for (-)-10c: $\boldsymbol{R}_{\boldsymbol{f}}=0.19\left(10 \%\right.$ EtOAc-hexane). $[\boldsymbol{\alpha}]^{\mathbf{2 0}}{ }_{\mathbf{D}}=-50.6(c=1.45) .{ }^{\mathbf{1}} \mathbf{H} \mathbf{N M R}(\mathbf{3 0 0} \mathbf{M H z}) \delta$ $0.14(\mathrm{~s}, 3 \mathrm{H}), 0.15$ (s, $3 \mathrm{H}), 0.96(\mathrm{~s}, 9 \mathrm{H}), 2.27$ (s, $3 \mathrm{H}), 3.87$ (ap t, $2 \mathrm{H}, J=4.2,3.6 \mathrm{~Hz}), 4.24$ (ap t, $1 \mathrm{H}, J$ = 4.6, 3.9 Hz), $4.36(\mathrm{~s}, 1 \mathrm{H}), 5.53(\mathrm{~s}, 1 \mathrm{H}), 6.95(\mathrm{~d}, 2 \mathrm{H}, J=8.1 \mathrm{~Hz}), 7.05-7.29(\mathrm{~m}, 5 \mathrm{H}), 7.31(\mathrm{~d}, 2 \mathrm{H}, J$ $=8.3 \mathrm{~Hz}) .{ }^{13} \mathrm{C}$ NMR (75 MHz) $\delta-5.5$ (2 C), 18.4, 21.5, 25.9 (3 C), 63.4, 65.7, 77.3, 77.5, 78.6, 128.1 
(2 C), 128.5 (2 C), 128.7 (2 C), 128.8, 129.1 (2 C), 133.4, 134.3, 144.9. IR (film): 3384, 3028, 2928, 1595, 1333, 1160, $1255 \mathrm{~cm}^{-1}$. MS (ES): $943[2 \mathrm{M}+\mathrm{Na}]^{+}, 483[\mathrm{M}+\mathrm{Na}]^{+}(100 \%), 478\left[\mathrm{M}+\mathrm{H}_{2} \mathrm{O}\right]^{+}, 461$ $[\mathrm{M}+1]^{+}$. Anal. calcd for $\mathrm{C}_{24} \mathrm{H}_{32} \mathrm{O}_{5} \mathrm{SSi}$ : C, 62.57; H, 7.00; S, 6.96. Found: C, 62.70; H, 6.84; S, 7.22.

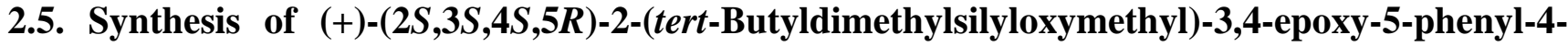
(p-tolylsulfonyl)tetrahydrofuran, $(+)-11 c$.

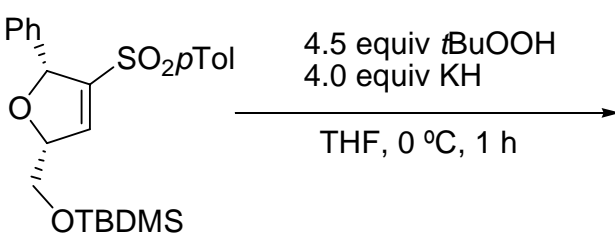

$(+)-9 \mathrm{c}$

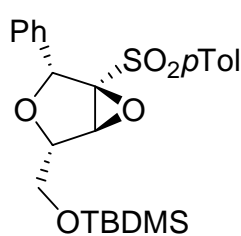

$(+)-11 c$

From a solution of sulfonyl dihydrofuran (+)-9c $(78 \mathrm{mg}, 0.175 \mathrm{mmol}, 1.0$ equiv) in $1.5 \mathrm{~mL}$ of THF, with $t$ BuOOK (4 equiv, in THF), according to the general procedure $\left(0{ }^{\circ} \mathrm{C}, 1 \mathrm{~h}\right)$, sulfonyl oxirane $(+)-$ 11c was obtained as a single isomer. Purification by chromatography (2-10\% EtOAc-hexane) gave (+)11c (49 $\mathrm{mg}, 61 \%)$ as a white solid and unreacted (+)-9c (8 mg, 10\%).

Data for (+)-11c: $\mathbf{m p :} 106-107{ }^{\circ} \mathrm{C} . \quad \boldsymbol{R}_{\boldsymbol{f}}=0.14(10 \%$ EtOAc-hexane $) . \quad[\alpha]^{20}{ }_{\mathbf{D}}=+66.8(c=1.08) .{ }^{1} \mathbf{H}$ NMR (300 MHz) $\delta 0.12$ (s, 3 H), 0.13 (s, 3 H), 0.94 (s, 9 H), 2.41 (s, 3 H), 3.90 (d, 2 H, J = 5.6 Hz), 4.19 (t, $1 \mathrm{H}, J=5.5 \mathrm{~Hz}), 4.43$ (s, $1 \mathrm{H}), 5.02$ (s, $1 \mathrm{H}), 7.21$ (d, $2 \mathrm{H}, J=8.3 \mathrm{~Hz}), 7.24-7.36$ (m, 5 H), 7.39 $(\mathrm{d}, 2 \mathrm{H}, J=8.1 \mathrm{~Hz}) .{ }^{13} \mathrm{C}$ NMR (75 MHz) $\delta-5.5(2 \mathrm{C}), 18.3,21.7,25.9$ (3 C), 63.1, 68.0, 78.8, 79.6, 82.4, 127.9 (2 C), 129.0 (2 C), 129.1 (2 C), 129.2, 129.6 (2 Cr), 133.9, 135.4, 145.5. IR (film): 3467, 2956, 2927, 1631, 1595, 1329, $1157 \mathrm{~cm}^{-1}$. MS (ES): $943[2 \mathrm{M}+\mathrm{Na}]^{+}, 483[\mathrm{M}+\mathrm{Na}]^{+}(100 \%), 478$ $\left[\mathrm{M}+\mathrm{H}_{2} \mathrm{O}\right]^{+}, 461[\mathrm{M}+1]^{+}$. Anal. calcd for $\mathrm{C}_{24} \mathrm{H}_{32} \mathrm{O}_{5} \mathrm{SSi}: \mathrm{C}, 62.57 ; \mathrm{H}, 7.00 ; \mathrm{S}, 6.96$. Found: $\mathrm{C}, 62.38 ; \mathrm{H}$, $7.20 ; \mathrm{S}, 6.84$.

\subsection{General Procedure for Deprotection of Silyl Ethers with $n \mathrm{Bu}_{4} \mathrm{NF} / \mathrm{AcOH}$.}

To a cold $\left(0^{\circ} \mathrm{C}\right)$ solution of the substrate in THF $(5 \mathrm{~mL} / \mathrm{mmol})$ was added $\mathrm{AcOH}$ (3.0 equiv) followed by $n \mathrm{Bu}_{4} \mathrm{NF}$ (1.8 equiv) and the mixture was allowed to warm up and monitored by TLC. Upon completion, the solvent was removed under reduced pressure and the residue was filtered through a short plug of silica gel and then purified by chromatography on silica gel using the appropriate mixture of solvents. 


\subsection{Synthesis of $(+)-(2 S, 3 S, 4 S, 5 R)-3,4-E p o x y-5-i s o p r o p y l-4-(p$-tolylsulfonyl)tetrahydrofuran-2-} methanol, (+)-4a.

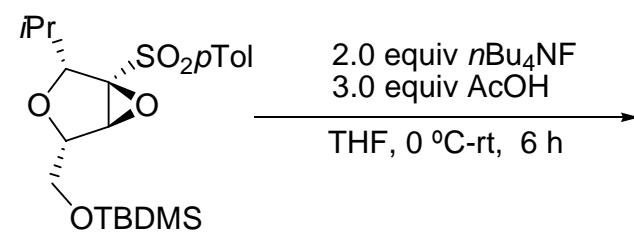

$(+)-11 a$

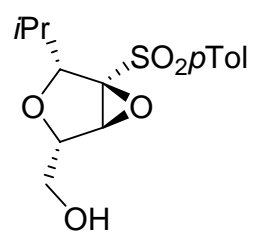

$(+)-4 a$

From (+)-11a (6 mg, $0.014 \mathrm{mmol}, 1.0$ equiv), with $n \mathrm{Bu}_{4} \mathrm{NF}(8.8 \mathrm{mg}, 0.028 \mathrm{mmol})$, following the general procedure $(6 \mathrm{~h})$ and after chromatography (20\% EtOAc-hexane), alcohol (+)-4a (4 mg, 92\%) was obtained. This product had identical spectral data to that found before for racemic material. ${ }^{2}$

Data for (+)-4a: $\boldsymbol{R}_{\boldsymbol{f}}=0.18\left(20 \%\right.$ EtOAc-hexane). $[\boldsymbol{\alpha}]^{20}{ }_{\mathbf{D}}=+19.7(c=0.38)$.

\subsection{Synthesis of (-)-(2R,3R,4S,5R)-3,4-Epoxy-5-phenyl-4-(p-tolylsulfonyl)tetrahydrofuran-2-} methanol, (-)-5c.

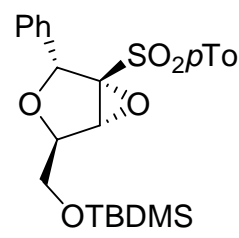

$(-)-10 c$

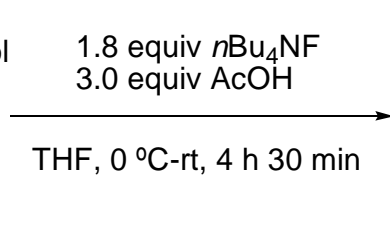

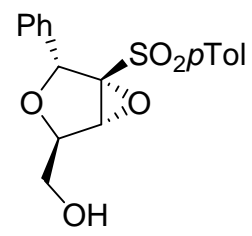

$(-)-5 c$

From (-)-10c (9 mg, $0.019 \mathrm{mmol}, 1.0$ equiv), with $n \mathrm{Bu}_{4} \mathrm{NF}(10.8 \mathrm{mg}, 0.034 \mathrm{mmol})$, following the general procedure (4 h $30 \mathrm{~min})$ and after chromatography (20\% EtOAc-hexane), alcohol (-)-5c (7.2 mg, $100 \%$ ) was obtained. This product had identical spectral data to that found before for racemic material. ${ }^{2}$

Data for $(-)-5 c: \boldsymbol{R}_{\boldsymbol{f}}=0.23\left(50 \%\right.$ EtOAc-hexane). $[\alpha]^{20}{ }_{\mathbf{D}}=-24.4(c=0.72)$.

\subsection{Synthesis of $(+)-(2 S, 3 S, 4 R, 5 R)-3,4-E p o x y-5-p h e n y l-4-(p$-tolylsulfonyl)tetrahydrofuran-2-} methanol, (+)-4c.

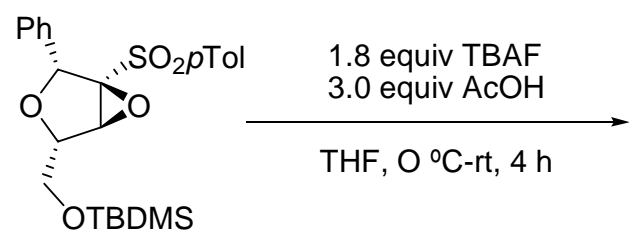

$(+)-11 c$

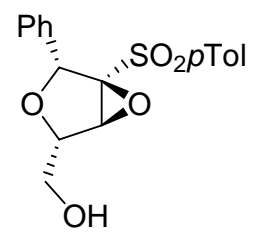

$(+)-4 c$

From (+)-11c (5 mg, $0.011 \mathrm{mmol}, 1.0$ equiv), with TBAF (6.2 mg, $0.020 \mathrm{mmol}, 1.8$ equiv), following the general procedure $(4 \mathrm{~h})$ and after chromatography $(20 \%$ EtOAc-hexane), alcohol (+)-4c $(3.8 \mathrm{mg}$, $100 \%$ ) was obtained. This product had identical spectral data to that found before for racemic material. ${ }^{2}$

Data for $(+)-4 c: \quad \boldsymbol{R}_{\boldsymbol{f}}=0.18(50 \%$ EtOAc-hexane $) .[\alpha]^{20}=+29.4(c=0.52)$. 


\subsection{General Procedure for Reductive Cleavage of Sulfinyl and Sulfonyl Oxiranes with (PhSe) and $\mathrm{NaBH}_{4}$.}

A two-necked round-bottomed flask was charged with a solution of $(\mathrm{PhSe})_{2}$ (0.6-5.0 equiv) in EtOH (3.5-6 $\mathrm{mL} / \mathrm{mmol}$ of diselenide) and argon was slowly bubbled through the solution with a pipette. To this solution was added powdered $\mathrm{NaBH}_{4}$ (5-14 equiv) portionwise at $\mathrm{rt}$ and the mixture was stirred until evolution of $\mathrm{H}_{2}$ subsided (ca. 1 h). In many cases $\mathrm{AcOH}$ ( 1 equiv) was added to ensure reproducibility and faster reaction rate. Then, a solution of 1 equiv of sulfinyl or sulfonyl oxirane in EtOH $(2-5 \mathrm{~mL} / \mathrm{mmol})$ was added and the reaction was monitored by TLC until disappearance of starting material. The reaction was then quenched with a saturated $\mathrm{NH}_{4} \mathrm{Cl}$ solution ( 3 drops $/ \mathrm{mmol}$ ), the solvent was removed under reduced pressure, the residue was taken up in EtOAc, filtered through a cotton plug and washed carefully with additional EtOAc. The solution was concentrated under reduced pressure to produce a crude product that was purified by chromatography on silica gel using the appropriate mixture of eluents, to produce substantial amounts $(70-80 \%)$ of the non polar $(\mathrm{PhSe})_{2}$ and the reaction products.

\subsection{Synthesis of ( \pm )-2-Ethoxy-2-isopropyl-3-oxotetrahydrofuran-5-methanol, 12a.}

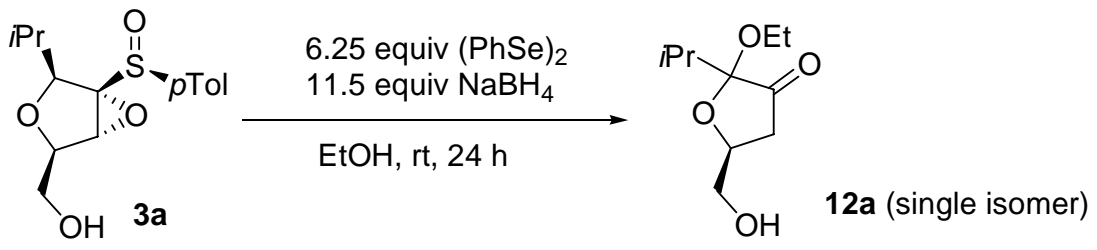

From a solution of epoxy sulfoxide $3 \mathbf{a}^{2}(50 \mathrm{mg}, 0.17 \mathrm{mmol}, 1.0$ equiv) in $1.7 \mathrm{~mL}$ of EtOH and a suspension generated from $(\mathrm{PhSe})_{2}\left(342 \mathrm{mg}, 1.1 \mathrm{mmol}, 6.25\right.$ equiv) in $5.4 \mathrm{~mL}$ of EtOH and $\mathrm{NaBH}_{4}(74$ $\mathrm{mg}, 1.95 \mathrm{mmol}, 11.5$ equiv), according to the general procedure (24 h) but without bubbling Argon through the mixture, a 67:33 mixture of hydroxy ketone 12a and starting material 3a was obtained. Purification by chromatography (5-75\% EtOAc-hexane) gave 12a (13 mg, 38\%) as a colorless oil and 3a $(8 \mathrm{mg}, 16 \%)$.

Data for 12a: $\quad \boldsymbol{R}_{\boldsymbol{f}}=0.17\left(50 \%\right.$ EtOAc-hexane). ${ }^{1} \mathbf{H}$ NMR (300 MHz) $\delta \quad 0.88(\mathrm{~d}, 3 \mathrm{H}, J=6.8 \mathrm{~Hz})$, $0.97(\mathrm{~d}, 3 \mathrm{H}, J=7.0 \mathrm{~Hz}), 1.16(\mathrm{t}, 3 \mathrm{H}, J=7.0 \mathrm{~Hz}), 2.22$ (ap sept, $1 \mathrm{H}, J=6.8 \mathrm{~Hz}), 2.49$ (dd, $1 \mathrm{H}, J=$ 18.8, 8.4 Hz), $2.68(\mathrm{dd}, 1 \mathrm{H}, J=18.8,5.0 \mathrm{~Hz}), 2.94(\mathrm{dd}, 1 \mathrm{H}, J=9.7,3.4 \mathrm{~Hz}), 3.42-3.61(\mathrm{~m}, 3 \mathrm{H}), 3.91$ $(\mathrm{dt}, 1 \mathrm{H}, J=12.3,2.7 \mathrm{~Hz}), 4.49(\mathrm{~m}, 1 \mathrm{H}) .{ }^{13} \mathbf{C} \mathbf{N M R}(50 \mathrm{MHz}) \delta 15.2,15.5,16.4,29.3,36.4,58.6$, 64.8, 75.5, 103.2, 210.0. IR $\left(\mathrm{CCl}_{4}\right): 3410,2950,2900,1730,1455,1370,1220,1180,1090,1020 \mathrm{~cm}^{-1}$. MS (EI): $203[\mathrm{M}+1]^{+}, 130,71,57,43$ (100\%). 


\subsection{Synthesis of ( \pm )-2-Ethoxy-4-phenylselenyl-2-isopropyl-3-oxotetrahydrofuran-5-methanol,} 13a.
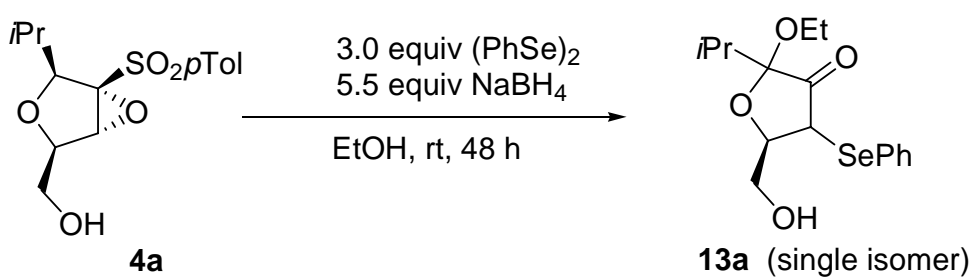

From a solution of epoxy sulfone $4 \mathrm{a}^{2}(48 \mathrm{mg}, 0.15 \mathrm{mmol}, 1.0$ equiv) in $1.0 \mathrm{~mL}$ of EtOH and a suspension generated from $(\mathrm{PhSe})_{2}\left(144 \mathrm{mg}, 0.46 \mathrm{mmol}, 3.0\right.$ equiv) in $3.0 \mathrm{~mL}$ of EtOH and $\mathrm{NaBH}_{4}(32$ $\mathrm{mg}, 0.85 \mathrm{mmol}$, 5.5 equiv), according to the general procedure (48 h) but without bubbling Argon through the mixture, a mixture of hydroxy ketone 13a and starting material 4a was obtained. Purification by chromatography (10-60\% EtOAc-hexane) gave 13a (3 mg, 5\%) as a colorless oil and 4a (17 mg, 35\%).

Data for 13a: $\boldsymbol{R}_{\boldsymbol{f}}=0.54\left(40 \%\right.$ EtOAc-hexane). ${ }^{1} \mathbf{H}$ NMR (200 MHz) $\delta \quad 0.74(\mathrm{~d}, 3 \mathrm{H}, J=6.8 \mathrm{~Hz})$, $0.90(\mathrm{~d}, 3 \mathrm{H}, J=6.8 \mathrm{~Hz}), 1.13(\mathrm{t}, 3 \mathrm{H}, J=7.0 \mathrm{~Hz}$ ), 1.94 (ap quint, $1 \mathrm{H}, J=6.8 \mathrm{~Hz}$ ), 2.68 (br s, $1 \mathrm{H}$ ), 3.38 (dq, $1 \mathrm{H}, J=8.8,7.0 \mathrm{~Hz}), 3.54$ (dq, $1 \mathrm{H}, J=8.8,7.1 \mathrm{~Hz}), 3.57-3.66$ (m, $1 \mathrm{H}), 3.91$ (dd, $1 \mathrm{H}, J=12.4$, $2.2 \mathrm{~Hz}), 4.01(\mathrm{~d}, 1 \mathrm{H}, J=6.5 \mathrm{~Hz}), 4.22(\mathrm{~m}, 1 \mathrm{H}), 7.26-7.32(\mathrm{~m}, 3 \mathrm{H}), 7.61-7.66(\mathrm{~m}, 2 \mathrm{H})$.

\subsection{Synthesis of $( \pm)$-2-Isopropyl-3-oxotetrahydrofuran-5-methanol, 14a and 15a.}

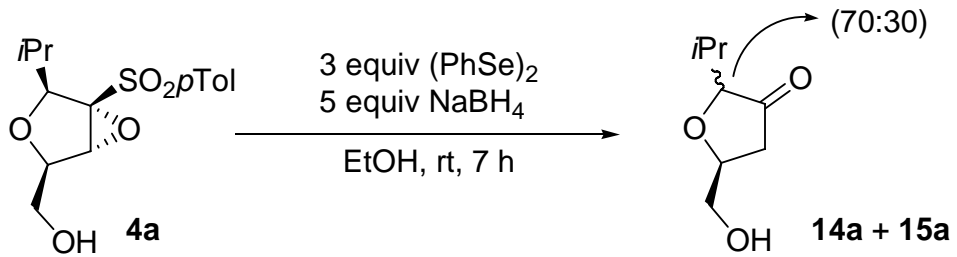

From a solution of epoxy sulfone $4 \mathrm{a}^{2}(62 \mathrm{mg}, 0.2 \mathrm{mmol}, 1.0$ equiv) in $0.6 \mathrm{~mL}$ of $\mathrm{EtOH}$ and a suspension generated from $(\mathrm{PhSe})_{2}\left(187 \mathrm{mg}, 0.6 \mathrm{mmol}, 3.0\right.$ equiv) in $2.4 \mathrm{~mL}$ of EtOH and $\mathrm{NaBH}_{4}(38$ $\mathrm{mg}, 1.0 \mathrm{mmol}, 5.0$ equiv), according to the general procedure $(7 \mathrm{~h})$, a 70:30 mixture of hydroxy ketones 14a and 15a was obtained along with small amounts of unreacted 4a and oxidation product 12a. Purification by chromatography (10-60\% EtOAc-hexane) gave an inseparable mixture of 14a and 15a $(26 \mathrm{mg}, 81 \%)$ as a colorless oil.

Data for 14a (from the mixture): $\boldsymbol{R}_{\boldsymbol{f}}=0.24$ (40\% EtOAc-hexane). ${ }^{1} \mathbf{H}$ NMR (200 MHz) $\delta 0.88(\mathrm{~d}, 3$ $\mathrm{H}, J=7.0 \mathrm{~Hz}), 1.02(\mathrm{~d}, 3 \mathrm{H}, J=7.0 \mathrm{~Hz}), 2.06(\mathrm{~m}, 1 \mathrm{H}), 2.40(\mathrm{~m}, 2 \mathrm{H}), 3.68(\mathrm{~m}, 2 \mathrm{H}), 3.95(\mathrm{dd}, 1 \mathrm{H}, J=$ 12.1, $2.7 \mathrm{~Hz}), 4.21(\mathrm{~m}, 1 \mathrm{H}) .{ }^{13} \mathbf{C}$ NMR (50 MHz) $\delta 16.8,18.7,30.1,39.3,63.7,75.5,85.6,215.3$. Anal. calcd for $\mathrm{C}_{8} \mathrm{H}_{14} \mathrm{O}_{3}: \mathrm{C}, 60.74 ; \mathrm{H}, 8.92$. Found: $\mathrm{C}, 60.45 ; \mathrm{H}, 9.03$. 
Partial data for 15a (from the mixture): $\boldsymbol{R}_{\boldsymbol{f}}=0.24$ (40\% EtOAc-hexane). ${ }^{1} \mathbf{H}$ NMR (200 MHz) $\delta$ $0.90(\mathrm{~d}, 3 \mathrm{H}, J=6.8 \mathrm{~Hz}), 1.00(\mathrm{~d}, 3 \mathrm{H}, J=7.0 \mathrm{~Hz}), 3.79$ (d, $1 \mathrm{H}, J=4.8 \mathrm{~Hz}), 4.45(\mathrm{~m}, 1 \mathrm{H}) .{ }^{13} \mathbf{C} \mathbf{N M R}$ (50 MHz) $\delta 17.1,18.7,30.8,38.4,64.7,75.9,84.1$.

\subsection{Synthesis of ( \pm )-2-Ethyl-3-oxotetrahydrofuran-5-methanol, $14 b$ and $15 b$.}

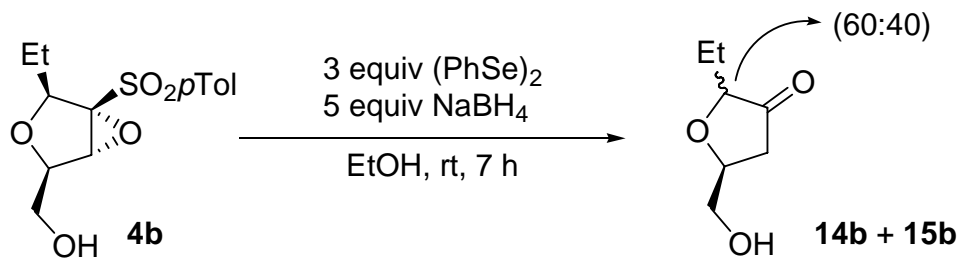

From a solution of epoxy sulfone $4 \mathbf{b}^{2}(358 \mathrm{mg}, 1.2 \mathrm{mmol}, 1.0$ equiv) in $2.4 \mathrm{~mL}$ of $\mathrm{EtOH}$ and a suspension generated from $(\mathrm{PhSe})_{2}\left(1.12 \mathrm{~g}, 3.6 \mathrm{mmol}, 3.0\right.$ equiv) in $12.6 \mathrm{~mL}$ of EtOH and $\mathrm{NaBH}_{4}(228$ $\mathrm{mg}, 6.0 \mathrm{mmol}, 5.0$ equiv), according to the general procedure $(7 \mathrm{~h})$, a 60:40 mixture of hydroxy ketones 14b and 15b was obtained. Purification by chromatography (10-20\% EtOAc-hexane) gave an inseparable mixture of $\mathbf{1 4 b}$ and $\mathbf{1 5 b}(143 \mathrm{mg}, 83 \%)$ as a colorless oil and $\mathbf{4 b}(34 \mathrm{mg}, 9 \%)$.

Data for 14b (from the mixture): $\boldsymbol{R}_{\boldsymbol{f}}=0.20$ (50\% EtOAc-hexane). ${ }^{\mathbf{1}} \mathbf{H}$ NMR (200 MHz) $\delta \quad 0.92(\mathrm{t}, 3$ H, $J=7.4 \mathrm{~Hz}), 1.60$ (m, $2 \mathrm{H}), 2.35$ (br s, $1 \mathrm{H}), 2.41$ (d, $2 \mathrm{H}, J=8.1 \mathrm{~Hz}), 3.64$ (dd, $1 \mathrm{H}, J=12.1,4.5 \mathrm{~Hz})$, $3.76(\mathrm{dd}, 1 \mathrm{H}, J=6.6,4.6 \mathrm{~Hz}), 3.87-3.97(\mathrm{~m}, 1 \mathrm{H}), 4.24(\mathrm{tdd}, 1 \mathrm{H}, J=8.1,4.5,2.8 \mathrm{~Hz}) .{ }^{13} \mathbf{C} \mathbf{N M R}(\mathbf{5 0}$ MHz) $\delta$ 9.4, 24.1, 38.4, 63.5, 75.7, 82.4, 215.5. IR (film): 3445, 2968, 2933, 2879, 1755 (strong), 1461, 1303, 1148, $667 \mathrm{~cm}^{-1}$. Anal. calcd for $\mathrm{C}_{7} \mathrm{H}_{12} \mathrm{O}_{3}: \mathrm{C}, 58.32 ; \mathrm{H}, 8.39$. Found: $\mathrm{C}, 58.64 ; \mathrm{H}, 8.56$.

Data for 15b (from the mixture): $\boldsymbol{R}_{\boldsymbol{f}}=0.20$ (50\% EtOAc-hexane). ${ }^{\mathbf{1}} \mathbf{H}$ NMR (200 MHz) $\delta \quad 0.94$ (t, 3 H, $J=7.4 \mathrm{~Hz}$ ), 1.73 (m, $2 \mathrm{H}), 2.35$ (br s, $1 \mathrm{H}), 2.47$ (d, $2 \mathrm{H}, J=7.1 \mathrm{~Hz}), 3.60$ (dd, $1 \mathrm{H}, J=11.9,4.7 \mathrm{~Hz}$ ), 3.83-3.96 (m, $2 \mathrm{H}), 4.42(\mathrm{tdd}, 1 \mathrm{H}, J=7.1,4.8,2.9 \mathrm{~Hz}) .{ }^{13} \mathbf{C}$ NMR (50 MHz) $\delta$ 9.5, 24.4, 37.8, 64.3, $75.5,80.7,215.9$.

\subsection{Synthesis of ( \pm )-2-phenyl-3-oxotetrahydrofuran-5-methanol, 14c and 15c.}
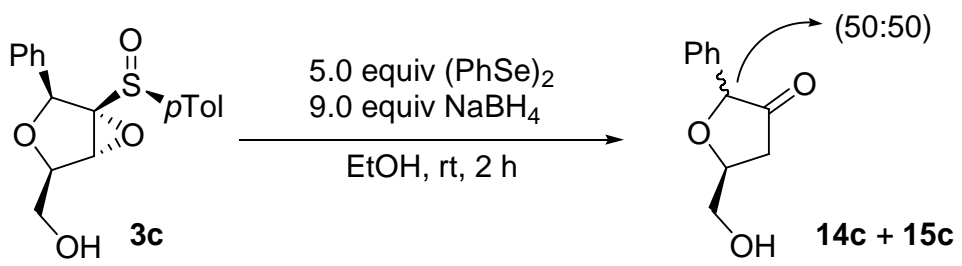

From a solution of epoxy sulfoxide $3 c^{2}(30 \mathrm{mg}, 0.09 \mathrm{mmol}, 1.0$ equiv) in $0.5 \mathrm{~mL}$ of EtOH and a suspension generated from $(\mathrm{PhSe})_{2}\left(141 \mathrm{mg}, 0.45 \mathrm{mmol}, 5.0\right.$ equiv) in $2.7 \mathrm{~mL}$ of EtOH and $\mathrm{NaBH}_{4}(31$ $\mathrm{mg}, 0.82 \mathrm{mmol}, 9.0$ equiv), according to the general procedure ( $2 \mathrm{~h})$, a 50:50 mixture of hydroxy ketones 14c and 15c was obtained. Purification by chromatography (10-80\% EtOAc-hexane) gave an 
inseparable mixture of $14 \mathrm{c}$ and $15 \mathrm{c}(10 \mathrm{mg}, 57 \%)$ as a colorless oil. The partial data listed below was obtained from enriched samples.

Partial data for 14c: $\boldsymbol{R}_{\boldsymbol{f}}=0.39$ (75\% EtOAc-hexane). ${ }^{1} \mathbf{H}$ NMR (200 MHz) $\delta 2.60(\mathrm{~m}, 2 \mathrm{H}), 3.72$ (dd, $1 \mathrm{H}, J=12.0,3.0 \mathrm{~Hz}), 3.98$ (dd, $1 \mathrm{H}, J=12.0,3.0 \mathrm{~Hz}), 4.67$ (m, $1 \mathrm{H}), 5.05$ (s, $1 \mathrm{H}), 7.28-7.39$ (m, 5 $\mathrm{H})$.

Partial data for 15c: $\boldsymbol{R}_{\boldsymbol{f}}=0.39$ (75\% EtOAc-hexane). ${ }^{1} \mathbf{H}$ NMR (200 MHz) $\delta 2.54(\mathrm{dd}, 1 \mathrm{H}, J=$ 17.2, $6.1 \mathrm{~Hz}), 2.70$ (dd, $1 \mathrm{H}, J=17.2,10.1 \mathrm{~Hz}), 3.78$ (dd, $1 \mathrm{H}, J=12.3,4.4 \mathrm{~Hz}), 4.06$ (dd, $1 \mathrm{H}, J=12.3$, $2.8 \mathrm{~Hz}), 4.45$ (m, $1 \mathrm{H}), 4.80$ (s, $1 \mathrm{H}), 7.28-7.39$ (m, 5 H).

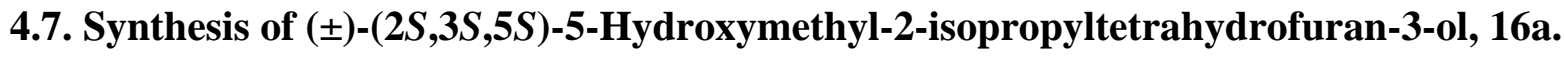

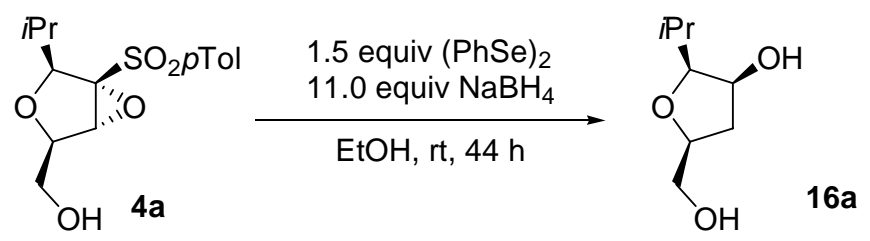

From a solution of epoxy sulfone $4 \mathbf{a}^{2}(400 \mathrm{mg}, 1.28 \mathrm{mmol}, 1.0$ equiv) in $3.8 \mathrm{~mL}$ of EtOH and a suspension generated from $(\mathrm{PhSe})_{2}\left(600 \mathrm{mg}, 1.92 \mathrm{mmol}, 1.5\right.$ equiv) in $9.6 \mathrm{~mL}$ of EtOH and $\mathrm{NaBH}_{4}(535$ $\mathrm{mg}, 1.46 \mathrm{mmol}, 11.0$ equiv), according to the general procedure $(44 \mathrm{~h})$, diol 16a was obtained as a single isomer. Purification by chromatography (20-80\% EtOAc-hexane) gave unreacted 4a (69 mg, $17 \%)$ and $16 a(115 \mathrm{mg}, 56 \%)$ as a white solid that was recrystallized from EtOAc-hexane.

Data of 16a: mp: $68-69{ }^{\circ} \mathrm{C} . \boldsymbol{R}_{\boldsymbol{f}}=0.16\left(60 \%\right.$ EtOAc-hexane). ${ }^{1} \mathbf{H}$ NMR (200 MHz) $\delta 0.92(\mathrm{~d}, 3 \mathrm{H}, J$ $=6.6 \mathrm{~Hz}), 1.02(\mathrm{~d}, 3 \mathrm{H}, J=6.6 \mathrm{~Hz}), 1.63(\mathrm{br} \mathrm{s}, 2 \mathrm{H}), 1.87$ (dd, $1 \mathrm{H}, J=14.1,3.1 \mathrm{~Hz}), 1.93-2.02(\mathrm{~m}, 1$ H), 2.35 (ddd, $1 \mathrm{H}, J=14.1,10.1,5.3 \mathrm{~Hz}$ ), 3.18 (dd, $1 \mathrm{H}, J=9.6,2.4 \mathrm{~Hz}$ ), 3.50 (dd, $1 \mathrm{H}, J=11.6,2.0$ Hz), 3.86 (dd, $1 \mathrm{H}, J=11.5,2.3 \mathrm{~Hz}$ ), 4.09 (dd, $1 \mathrm{H}, J=5.3,2.4 \mathrm{~Hz}$ ), 4.17 (dddd, $1 \mathrm{H}, J=10.1,5.3$, 3.1, $2.3 \mathrm{~Hz}) .{ }^{13} \mathrm{C}$ NMR (50 MHz) $\delta$ 18.8, 20.4, 27.7, 37.5, 64.4, 70.8, 77.5, 90.6. IR (KBr): 3369, 2951, 2921, 2874, 1455, 1287, 1082, 1047, 1026, 971, 838, $727 \mathrm{~cm}^{-1}$. MS (ES): $161[\mathrm{M}+1]^{+}$. Anal. calcd for $\mathrm{C}_{8} \mathrm{H}_{16} \mathrm{O}_{3}:$ C, 59.98; H, 10.07. Found: C, 59.68; H, 9.87. 


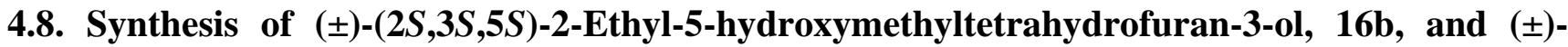

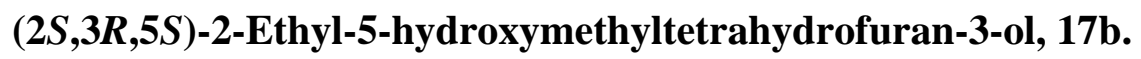
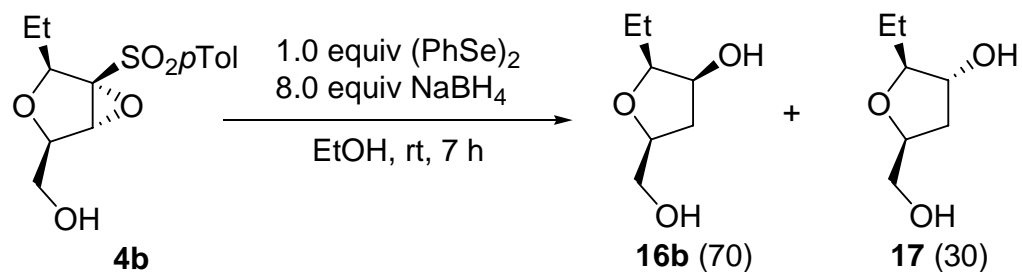

From a solution of epoxy sulfone $4 \mathbf{b}^{2}(167 \mathrm{mg}, 0.56 \mathrm{mmol}, 1.0$ equiv) in $1.1 \mathrm{~mL}$ of EtOH and a suspension generated from $(\mathrm{PhSe})_{2}\left(175 \mathrm{mg}, 0.56 \mathrm{mmol}, 1.0\right.$ equiv) in $2.8 \mathrm{~mL}$ of EtOH and $\mathrm{NaBH}_{4}(170$ $\mathrm{mg}, 4.48 \mathrm{mmol}, 8.0$ equiv), according to the general procedure $(7 \mathrm{~h})$, a 70:30 mixture of diols $\mathbf{1 6 b}$ and 17b was obtained. Purification by chromatography (30-100\% EtOAc-hexane) gave $16 \mathbf{b}$ (34 mg, 41\%) and $\mathbf{1 7 b}(14 \mathrm{mg}, 18 \%)$ as colorless oils.

Data of 16b: $\boldsymbol{R}_{\boldsymbol{f}}=0.23$ (80\% EtOAc-hexane). ${ }^{1} \mathbf{H}$ NMR (300 MHz) $\delta 0.94(\mathrm{t}, 3 \mathrm{H}, J=7.5 \mathrm{~Hz}), 1.64$ (m, $2 \mathrm{H}), 1.87(\mathrm{dd}, 1 \mathrm{H}, J=14.1,3.3 \mathrm{~Hz}), 1.87$ (s, $1 \mathrm{H}), 2.33$ (ddd, $1 \mathrm{H}, J=15.2,10.0,5.1 \mathrm{~Hz}), 3.82$ (br s, $1 \mathrm{H}), 3.46(\mathrm{dd}, 1 \mathrm{H}, J=11.9,2.2 \mathrm{~Hz}), 3.55(\mathrm{dd}, 1 \mathrm{H}, J=7.0,2.4 \mathrm{~Hz}), 3.81(\mathrm{dd}, 1 \mathrm{H}, J=11.7,2.4 \mathrm{~Hz})$, 4.01 (br s, $1 \mathrm{H}), 4.16$ (m, 1 H). ${ }^{13}$ C NMR (50 MHz) $\delta \quad 10.4,21.7,37.1,63.9,71.0,77.1,86.0$. IR (film): 3369, 2963, 2933, 2876, 1651, 1462, 1184, 1095, 1040, 975, $867 \mathrm{~cm}^{-1}$. MS (ES): $147[\mathrm{M}+1]^{+}$ (100\%). Anal. calcd for $\mathrm{C}_{7} \mathrm{H}_{14} \mathrm{O}_{3}: \mathrm{C}, 57.51 ; \mathrm{H}, 9.65$. Found: $\mathrm{C}, 57.82 ; \mathrm{H}, 9.50$.

Data of 17b: $\quad \boldsymbol{R}_{\boldsymbol{f}}=0.10$ (80\% EtOAc-hexane). ${ }^{1} \mathbf{H}$ NMR (300 MHz) $\delta 0.97(\mathrm{t}, 3 \mathrm{H}, J=7.4 \mathrm{~Hz})$, 1.47-1.60 (m, $4 \mathrm{H}), 1.82$ (ddd, $1 \mathrm{H}, J=13.2,6.2,2.7 \mathrm{~Hz}), 1.99$ (ddd, $1 \mathrm{H}, J=13.2,9.4,6.3 \mathrm{~Hz}$ ), 3.48 $(\mathrm{m}, 1 \mathrm{H}), 3.68(\mathrm{td}, 1 \mathrm{H}, J=6.5,3.3 \mathrm{~Hz}), 3.78(\mathrm{~m}, 1 \mathrm{H}), 4.08(\mathrm{~m}, 1 \mathrm{H}), 4.23(\mathrm{~m}, 1 \mathrm{H}) .{ }^{13} \mathbf{C}$ NMR (50 MHz) $\delta$ 10.3, 27.2, 36.3, 64.5, 76.2, 78.2, 88.3. IR (film): 3368, 2930, 1655, 1462, $1039 \mathrm{~cm}^{-1}$. MS (ES): $147[\mathrm{M}+1]^{+}(100 \%)$. Anal. calcd for $\mathrm{C}_{7} \mathrm{H}_{14} \mathrm{O}_{3}$ : C, 57.51; H, 9.65. Found: C, 57.63; H, 9.73.

4.9. Synthesis of $( \pm)-(2 S, 3 S, 5 S)-2-P h e n y l-5-h y d r o x y m e t h y l t e t r a h y d r o f u r a n-3-o l, 16 c$, and $( \pm)-$ (2S,3R,5S)-2-Phenyl-5-hydroxymethyltetrahydrofuran-3-ol, 17c.
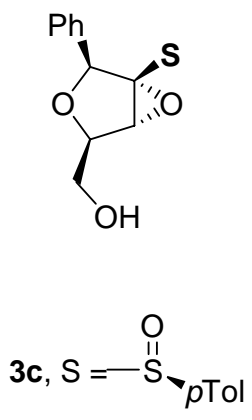

4c, $\mathrm{S}=-\mathrm{SO}_{2} \mathrm{pTol}$
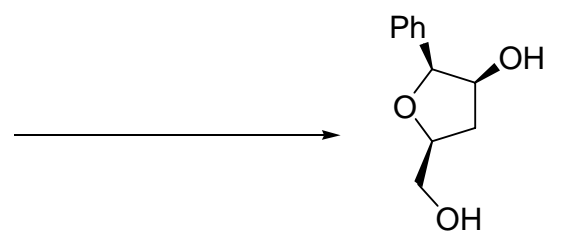

$16 \mathrm{c}$

80

5.0 equiv $\mathrm{NaBH}_{4}$

$\mathrm{EtOH}, 0^{\circ} \mathrm{C}, 1.5 \mathrm{~h}$

1.5 equiv ( $\mathrm{PhSe})_{2}$ 11.0 equiv $\mathrm{NaBH}_{4}$ $\mathrm{EtOH}, \mathrm{rt}, 17 \mathrm{~h}$

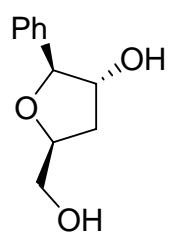

17c

20

20 
From a solution of epoxy sulfoxide $3 \mathrm{c}^{2}(120 \mathrm{mg}, 0.36 \mathrm{mmol}, 1.0$ equiv) in $0.7 \mathrm{~mL}$ of EtOH and a suspension generated from $(\mathrm{PhSe})_{2}\left(68 \mathrm{mg}, 0.22 \mathrm{mmol}, 0.6\right.$ equiv) in $1.1 \mathrm{~mL}$ of EtOH and $\mathrm{NaBH}_{4}(69$ $\mathrm{mg}, 1.81 \mathrm{mmol}, 5.0$ equiv), according to the general procedure (1 h $30 \mathrm{~min})$, an 80:20 mixture of diols 16c and 17c was obtained. Purification by chromatography (40-80\% EtOAc-hexane) gave 16c (46 mg, $66 \%)$ and 17c (12 mg, 17\%) as colorless oils.

In a related experiment, from a solution of epoxy sulfone 4c (46 mg, $0.13 \mathrm{mmol}, 1.0$ equiv) in $0.4 \mathrm{~mL}$ of EtOH and a suspension generated from $(\mathrm{PhSe})_{2}(62 \mathrm{mg}, 0.20 \mathrm{mmol}, 1.5$ equiv) in $1.0 \mathrm{~mL}$ of EtOH and $\mathrm{NaBH}_{4}$ (56 mg, $1.46 \mathrm{mmol}, 11.0$ equiv), according to the general procedure (17 h), an 80:20 mixture of diols 16c and 17c was obtained. Purification by chromatography (40-80\% EtOAc-hexane) gave 16c (13 mg, 51\%) and 17c (3 mg, 11\%) as colorless oils.

Data of 16c: $\quad \boldsymbol{R}_{\boldsymbol{f}}=0.25$ (80\% EtOAc-hexane). ${ }^{1} \mathbf{H}$ NMR (300 MHz) $\delta 2.03(\mathrm{dd}, 1 \mathrm{H}, J=14.0,3.6$ Hz), 2.47 (ddd, $1 \mathrm{H}, J=14.0,9.9,5.2 \mathrm{~Hz}$ ), 2.69 (br s, $2 \mathrm{H}), 3.63$ (dd, $1 \mathrm{H}, J=11.5,3.2 \mathrm{~Hz}$ ), 3.92 (dd, 1 $\mathrm{H}, J=11.5,2.5 \mathrm{~Hz}), 4.28(\mathrm{dd}, 1 \mathrm{H}, J=5.2,2.9 \mathrm{~Hz}), 4.35(\mathrm{ddd}, 1 \mathrm{H}, J=9.9,6.3,3.3 \mathrm{~Hz}), 4.85(\mathrm{~d}, 1 \mathrm{H}, J$ $=2.8 \mathrm{~Hz}), 7.25-7.40(\mathrm{~m}, 5 \mathrm{H}) .{ }^{13} \mathbf{C}$ NMR (50 MHz) $\delta 36.6,64.8,73.3,78.1,85.7,126.8(2 \mathrm{C}), 127.9$, 128.4 (2 C), 136.6. IR (film): 3399, 2926, 1649, 1452, 1044, 755, $700 \mathrm{~cm}^{-1}$. MS (ES): $217[\mathrm{M}+\mathrm{Na}]^{+}$ $(100 \%), 195[\mathrm{M}+1]^{+}$. Anal. calcd for $\mathrm{C}_{11} \mathrm{H}_{14} \mathrm{O}_{3}$ : C, 68.02; H, 7.27. Found: C, 67.69; H, 7.53.

Data of 17c: $\quad \boldsymbol{R}_{\boldsymbol{f}}=0.19$ (80\% EtOAc-hexane). ${ }^{1} \mathbf{H}$ NMR (200 MHz) $\delta 1.94$ (br s, $\left.2 \mathrm{H}\right), 1.94$ (ddd, 1 $\mathrm{H}, J=13.1,6.6,3.8 \mathrm{~Hz}), 2.13(\mathrm{ddd}, 1 \mathrm{H}, J=13.1,8.6,6.8 \mathrm{~Hz}), 3.65$ (m, $\left.1 \mathrm{H}, \mathrm{CH}_{2}-\mathrm{OH}\right), 3.88(\mathrm{~m}, 1 \mathrm{H})$, 4.27 (ap. dt, $1 \mathrm{H}, J=7.0,3.8 \mathrm{~Hz}), 4.41$ (m, $1 \mathrm{H}), 4.73(\mathrm{~d}, 1 \mathrm{H}, J=4.4 \mathrm{~Hz}), 7.25-7.38(\mathrm{~m}, 5 \mathrm{H}) .{ }^{13} \mathrm{C}$ NMR (50 MHz) $\delta$ 36.1, 64.7, 78.7 (2 C), 88.1, 125.9 (2 C), 127.9, 128.6 (2 C), 140.2. IR (film): 3390, 2927, 1603, 1452, 1337, 1280, 1045, $701 \mathrm{~cm}^{-1}$. MS (ES): $195[\mathrm{M}+1]^{+}(100 \%), 177\left[\mathrm{M}-\mathrm{H}_{2} \mathrm{O}\right]^{+}$. Anal. calcd for $\mathrm{C}_{11} \mathrm{H}_{14} \mathrm{O}_{3}: \mathrm{C}, 68.02 ; \mathrm{H}, 7.27$. Found: $\mathrm{C}, 68.26 ; \mathrm{H}, 7.42$.

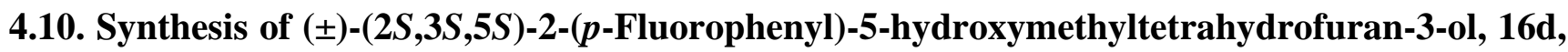

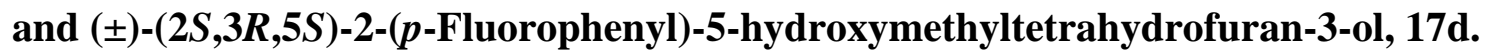

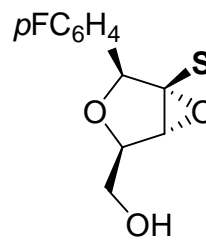

3d, $S=\stackrel{\text { II }}{\text { S }}$-pTol

$\mathbf{4 d}, \mathrm{S}=-\mathrm{SO}_{2} p \mathrm{Tol}$

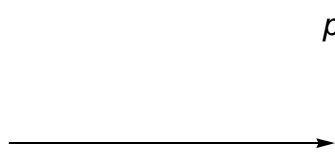

0.6 equiv $(\mathrm{PhSe})_{2}$ 5.0 equiv $\mathrm{NaBH}_{4}$ $\mathrm{EtOH}, 0{ }^{\circ} \mathrm{C}, 1.5 \mathrm{~h}$ 1.5 equiv $(\mathrm{PhSe})_{2}$ 11.0 equiv $\mathrm{NaBH}_{4}$ $\mathrm{EtOH}, \mathrm{rt}, 2.5 \mathrm{~h}$

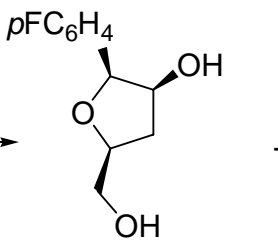

16d

86

85

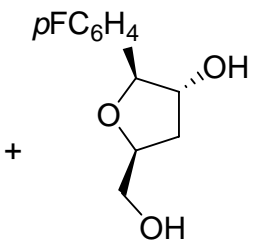

17d

14

15 
From a solution of epoxy sulfoxide $3 \mathbf{d}^{2}(52 \mathrm{mg}, 0.15 \mathrm{mmol}, 1.0$ equiv) in $0.6 \mathrm{~mL}$ of EtOH and a suspension generated from $(\mathrm{PhSe})_{2}\left(28 \mathrm{mg}, 0.09 \mathrm{mmol}, 0.6\right.$ equiv) in $0.75 \mathrm{~mL}$ of EtOH and $\mathrm{NaBH}_{4}(29$ $\mathrm{mg}, 0.75 \mathrm{mmol}, 5$ equiv), according to the general procedure $(1.5 \mathrm{~h})$, an 86:14 mixture of diols $\mathbf{1 6 d}$ and 17d was obtained. Purification by chromatography (20-80\% EtOAc-hexane) gave 16d (26 mg, 81\%) and 17d (4 mg, 13\%) as colorless oils.

In a related experiment from a solution of epoxy sulfone $4 \mathbf{d}^{2}(1.57 \mathrm{~g}, 4.3 \mathrm{mmol}, 1.0$ equiv) in $8.0 \mathrm{~mL}$ of EtOH and a suspension generated from $(\mathrm{PhSe})_{2}(2.01 \mathrm{~g}, 6.45 \mathrm{mmol}, 1.5$ equiv) in $26 \mathrm{~mL}$ of $\mathrm{EtOH}$ and $\mathrm{NaBH}_{4}$ (1.80 g, $47.3 \mathrm{mmol}, 11$ equiv), according to the general procedure $(2.5 \mathrm{~h})$, an 85:15 mixture of diols 16d and 17d was obtained. Purification by chromatography (40-80\% EtOAc-hexane) gave 16d (698 $\mathrm{mg}, 77 \%)$ and $\mathbf{1 7 d}(123 \mathrm{mg}, 13 \%)$ as colorless oils.

Data of 16d: $\boldsymbol{R}_{\boldsymbol{f}}=0.13\left(50 \%\right.$ EtOAc- $\left.\mathrm{CH}_{2} \mathrm{Cl}_{2}\right) .{ }^{1} \mathbf{H}$ NMR (300 MHz) $\delta 2.04(\mathrm{ddd}, 1 \mathrm{H}, J=14.0,3.4$, $0.9 \mathrm{~Hz}), 2.47$ (ddd, $1 \mathrm{H}, J=14.0,9.4,5.2 \mathrm{~Hz}), 2.90$ (br s, $2 \mathrm{H}), 3.60$ (dd, $1 \mathrm{H}, J=11.5,3.0 \mathrm{~Hz}), 3.91$ (dd, $1 \mathrm{H}, J=11.5,2.4 \mathrm{~Hz}), 4.21(\mathrm{dd}, 1 \mathrm{H}, J=4.9,2.9 \mathrm{~Hz}), 4.33(\mathrm{ddd}, 1 \mathrm{H}, J=9.9,5.7,3.1 \mathrm{~Hz}), 4.80$ (d, $1 \mathrm{H}, J=2.7 \mathrm{~Hz}), 7.00-7.07(\mathrm{~m}, 2 \mathrm{H}), 7.32-7.37(\mathrm{~m}, 2 \mathrm{H}) .{ }^{13} \mathbf{C}$ NMR (75 MHz) $\delta 36.7,64.5,73.0,77.8$, 85.1, $115.2\left(2 \mathrm{C}, J_{C-F}=21.2 \mathrm{~Hz}\right), 128.5\left(2 \mathrm{C}, J_{C-F}=8.1 \mathrm{~Hz}\right), 132.4,162.3\left(J_{C-F}=245.8 \mathrm{~Hz}\right)$. IR (film): 3350, 2938, 1606, 1512, 1445, 1294, 1222, 1157, 1118, 1059, 927, 877, 850, 808, $777 \mathrm{~cm}^{-1}$. MS (ES): $235[\mathrm{M}+\mathrm{Na}]^{+}(100 \%), 230,213[\mathrm{M}+1]^{+}$. Anal. calcd for $\mathrm{C}_{11} \mathrm{H}_{13} \mathrm{FO}_{3}: \mathrm{C}, 62.26 ; \mathrm{H}, 6.17$. Found: $\mathrm{C}$, $62.45 ; \mathrm{H}, 6.46$.

Data of 17d: $\boldsymbol{R}_{\boldsymbol{f}}=0.08\left(50 \%\right.$ EtOAc- $\left.\mathrm{CH}_{2} \mathrm{Cl}_{2}\right) .{ }^{\mathbf{1}} \mathbf{H}$ NMR (200 MHz) $\delta 1.92(\mathrm{ddd}, 1 \mathrm{H}, J=13.0,6.8$, $3.8 \mathrm{~Hz}$ ), 1.94 (br s, 2 H), 2.09 (ddd, $1 \mathrm{H}, J=13.2,8.4,6.8 \mathrm{~Hz}$ ), 3.64 (dd, $1 \mathrm{H}, J=11.9,5.3 \mathrm{~Hz}$ ), 3.86 (dd, $1 \mathrm{H}, J=11.9,2.9 \mathrm{~Hz}), 4.20$ (ap dt, $1 \mathrm{H}, J=7.0,4.1 \mathrm{~Hz}), 4.37$ (m, $1 \mathrm{H}), 4.69$ (d, $1 \mathrm{H}, J=4.4 \mathrm{~Hz}$ ), $7.02($ ap t, $2 \mathrm{H}, J=8.7 \mathrm{~Hz}), 7.32(\mathrm{ap} \mathrm{dd}, 2 \mathrm{H}, J=8.4,5.3 \mathrm{~Hz}) .{ }^{13} \mathbf{C} \mathbf{N M R}(\mathbf{5 0} \mathbf{M H z}) \delta 36.3,64.7,78.6$, 77.8, 87.5, $115.4\left(2 \mathrm{C}, J_{C-F}=21.5 \mathrm{~Hz}\right), 127.6\left(2 \mathrm{C}, J_{C-F}=8.0 \mathrm{~Hz}\right), 136.0,162.5\left(J_{C-F}=246.0 \mathrm{~Hz}\right)$. IR (film): 3368, 2928, 1606, 1511, 1225, 1045, 832, $757 \mathrm{~cm}^{-1}$. MS (ES): $235[\mathrm{M}+\mathrm{Na}]^{+}(100 \%), 213$ $[\mathrm{M}+1]^{+}$. Anal. calcd for $\mathrm{C}_{11} \mathrm{H}_{13} \mathrm{FO}_{3}$ : C, 62.26; H, 6.17. Found: $\mathrm{C}, 62.54 ; \mathrm{H}, 6.33$.

\subsection{Synthesis of $( \pm)-(2 S, 3 S, 5 S)-5-H y d r o x y m e t h y l-2-(3-p y r i d y l) t e t r a h y d r o f u r a n-3-o l, 16 e$, and} ( \pm )-(2S,3R,5S)-5-Hydroxymethyl-2-(3-pyridyl)tetrahydrofuran-3-ol, 16 e.

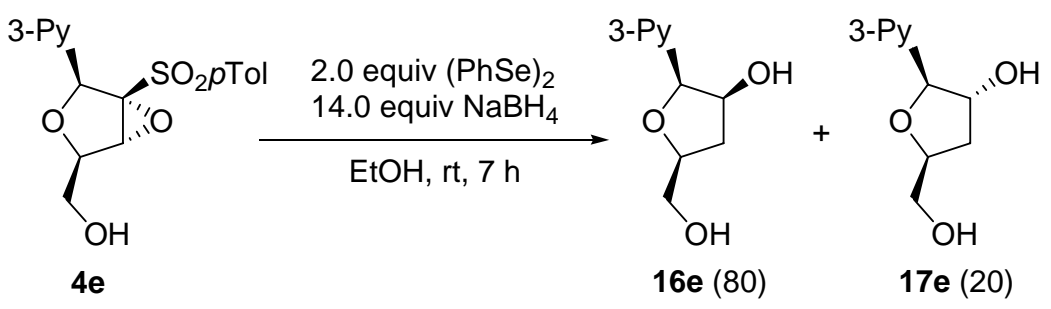


From a solution of epoxy sulfone $4 \mathrm{e}^{2}(56 \mathrm{mg}, 0.16 \mathrm{mmol}, 1.0$ equiv) in $0.5 \mathrm{~mL}$ of EtOH and a suspension generated from $(\mathrm{PhSe})_{2}\left(101 \mathrm{mg}, 0.32 \mathrm{mmol}, 2.0\right.$ equiv) in $1.6 \mathrm{~mL}$ of EtOH and $\mathrm{NaBH}_{4}(86$ $\mathrm{mg}, 2.26 \mathrm{mmol}, 14.0$ equiv), according to the general procedure (7 h), an 80:20 mixture of diols 16e and 17e was obtained. Purification by chromatography (0-20\% MeOH-EtOAc) gave an inseparable mixture of 16e and 17e (16 mg, 51\%) as a colorless oil.

Data of 16e (from the mixture): $\boldsymbol{R}_{\boldsymbol{f}}=0.18(20 \% \mathrm{MeOH}-\mathrm{EtOAc}) .{ }^{1} \mathbf{H}$ NMR (200 MHz) $\delta 2.16(\mathrm{dd}, 1$ $\mathrm{H}, J=13.9,2.7 \mathrm{~Hz}), 2.56(\mathrm{ddd}, 1 \mathrm{H}, J=13.9,10.1,5.1 \mathrm{~Hz}), 3.62(\mathrm{dd}, 1 \mathrm{H}, J=11.7,2.0 \mathrm{~Hz}), 4.01$ (dd, 1 $\mathrm{H}, J=11.7,2.0 \mathrm{~Hz}), 4.26(\mathrm{dd}, 1 \mathrm{H}, J=4.9,2.9 \mathrm{~Hz}), 4.40(\mathrm{ap} \mathrm{dq}, 1 \mathrm{H}, J=10.2,2.4 \mathrm{~Hz}), 4.86(\mathrm{~d}, 1 \mathrm{H}, J=$ $2.8 \mathrm{~Hz}), 7.23-7.29(\mathrm{~m}, 1 \mathrm{H}), 7.69(\mathrm{~m}, 1 \mathrm{H}), 8.40(\mathrm{dd}, 1 \mathrm{H}, J=4.9,1.5 \mathrm{~Hz}), 8.60(\mathrm{~d}, 1 \mathrm{H}, J=2.0 \mathrm{~Hz})$. ${ }^{13}$ C NMR (50 MHz) $\delta$ 37.4, 64.1, 72.9, 78.2, 84.2, 123.3, 135.6 (2 C), 147.9, 148.4. IR (film): 3350, 2929, 1599, 1430, 1191, 1029, 758, $712 \mathrm{~cm}^{-1}$. MS (APCI): $196[\mathrm{M}+1]^{+}(100 \%)$. Anal. calcd for $\mathrm{C}_{10} \mathrm{H}_{13} \mathrm{NO}_{3}$ : C, 61.53; H, 6.71. Found: C, 61.18; H, 6.97.

Partial data of 17e (from the mixture): $\boldsymbol{R}_{\boldsymbol{f}}=0.18$ (20\% MeOH-EtOAc). ${ }^{\mathbf{1}} \mathbf{H}$ NMR (200 MHz) $\delta 1.91$ (m, $1 \mathrm{H}), 2.28(\mathrm{ddd}, 1 \mathrm{H}, J=13.7,9.4,5.9 \mathrm{~Hz}), 4.52(\mathrm{~m}, 1 \mathrm{H}), 5.07$ (s, $1 \mathrm{H}), 7.61(\mathrm{~m}, 1 \mathrm{H}), 8.46(\mathrm{dd}, 1$ $\mathrm{H}, J=4.8,1.6 \mathrm{~Hz}), 8.55(\mathrm{~d}, 1 \mathrm{H}, J=2.2 \mathrm{~Hz})$.

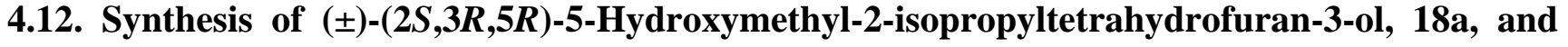
( \pm )-(2S,3S,5R)-5-Hydroxymethyl-2-isopropyltetrahydrofuran-3-ol, 19a.

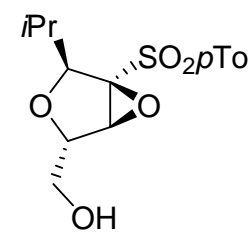

$5 a$

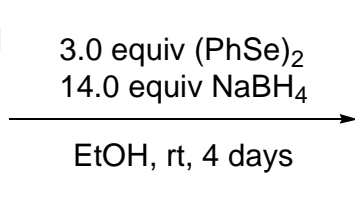

EtOH, rt, 4 days

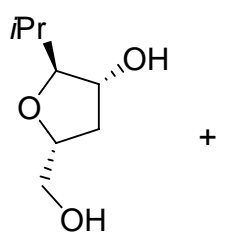

$18 \mathrm{a}(58)$

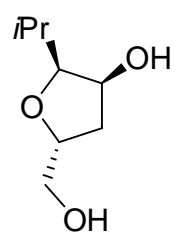

$19 \mathrm{a}(42)$

From a solution of epoxy sulfone $5 \mathrm{a}^{2}(165 \mathrm{mg}, 0.53 \mathrm{mmol}, 1.0$ equiv) in $1.6 \mathrm{~mL}$ of EtOH and a suspension generated from $\left(\mathrm{PhSe}_{2}\left(495 \mathrm{mg}, 1.56 \mathrm{mmol}, 3.0\right.\right.$ equiv) in $8.0 \mathrm{~mL}$ of EtOH and $\mathrm{NaBH}_{4}(281$ $\mathrm{mg}, 7.39 \mathrm{mmol}, 14.0$ equiv), according to the general procedure (4 days), a 58:42 mixture of diols 18a and 19a was obtained. Purification by chromatography (30-100\% EtOAc-hexane) gave 18a (21 mg, 25\%), 19a (20 mg, 23\%) as colorless oils along with unreacted 5 a (17 mg, 10\%).

Data of 18a: $\boldsymbol{R}_{\boldsymbol{f}}=0.22(\mathrm{EtOAc}) .{ }^{1} \mathbf{H}$ NMR (300 MHz) $\delta 0.89(\mathrm{~d}, 3 \mathrm{H}, J=6.7 \mathrm{~Hz}), 0.92(\mathrm{~d}, 3 \mathrm{H}, J=$ $6.6 \mathrm{~Hz}$ ), 1.53 (dhept, $1 \mathrm{H}, J=8.0,6.7 \mathrm{~Hz}$ ), 1.80 (dddd, $1 \mathrm{H}, J=13.9,3.7,2.0,0.7 \mathrm{~Hz}$ ), 2.30 (ddd, $1 \mathrm{H}, J$ = 13.9, 9.2, $6.2 \mathrm{~Hz}), 3.04$ (br s, $1 \mathrm{H}), 3.51(\mathrm{dd}, 1 \mathrm{H}, J=11.6,2.6 \mathrm{~Hz}), 3.61$ (dd, $1 \mathrm{H}, J=8.0,1.8 \mathrm{~Hz})$, 3.69 (s, $1 \mathrm{H}), 3.80$ (dd, $1 \mathrm{H}, J=11.6,2.5 \mathrm{~Hz}), 4.14$ (m, $1 \mathrm{H}), 4.19$ (dddd, $1 \mathrm{H}, J=9.3,6.5,3.7,2.8 \mathrm{~Hz}$ ). ${ }^{13}$ C NMR (50 MHz) $\delta 18.7,18.8,30.9,36.7,64.9,73.6,77.6,93.5$. IR $\left(\mathrm{CCl}_{4}\right): 3435,2925,1628$, $1457,1279 \mathrm{~cm}^{-1}$. Anal. calcd for $\mathrm{C}_{8} \mathrm{H}_{16} \mathrm{O}_{3}: \mathrm{C}, 59.98 ; \mathrm{H}, 10.07$. Found: $\mathrm{C}, 60.29 ; \mathrm{H}, 9.75$. 
Data of 19a: $\boldsymbol{R}_{\boldsymbol{f}}=0.26(\mathrm{EtOAc}) .{ }^{1} \mathbf{H}$ NMR (300 MHz) $\delta 0.91(\mathrm{~d}, 3 \mathrm{H}, J=6.6 \mathrm{~Hz}), 1.03(\mathrm{~d}, 3 \mathrm{H}, J=$ $6.7 \mathrm{~Hz}), 1.60(\mathrm{~m}, 1 \mathrm{H}), 1.83-2.02(\mathrm{~m}, 3 \mathrm{H}), 2.00$ (br s, $1 \mathrm{H}), 3.30$ (dd, $1 \mathrm{H}, J=9.8,2.6 \mathrm{~Hz}), 3.46$ (m, 1 H), $3.65(\mathrm{~m}, 1 \mathrm{H}), 4.29(\mathrm{~m}, 1 \mathrm{H}), 4.32(\mathrm{ddd}, 1 \mathrm{H}, J=9.8,6.0,3.2 \mathrm{~Hz}) .{ }^{13} \mathbf{C} \mathbf{N M R}(50 \mathrm{MHz}) \delta 18.9$, 20.4, 28.0, 37.3, 65.0, 72.5, 77.6, 88.7. IR (film): 3391, 2959, 2873, 1650, 1470, 1388, 1367, 1334, 1181, 1133, 1036, $756 \mathrm{~cm}^{-1}$. MS (ES): $183[\mathrm{M}+\mathrm{Na}]^{+}(100 \%), 161[\mathrm{M}+1]^{+}$. Anal. calcd for $\mathrm{C}_{8} \mathrm{H}_{16} \mathrm{O}_{3}$ : C, 59.98; H, 10.07. Found: C, 59.64; H, 10.23.

\subsection{Synthesis of $( \pm)-(2 S, 3 R, 5 R)-2-(p$-Fluorophenyl)-5-hydroxymethyltetrahydrofuran-3-ol,} 18d, and ( \pm )-(2S,3S,5R)-2-(p-Fluorophenyl)-5-hydroxymethyltetrahydrofuran-3-ol, $19 \mathrm{~d}$.

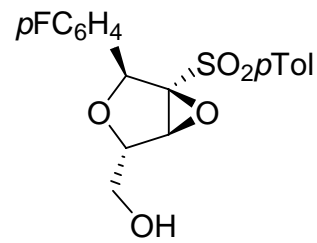

$5 d$

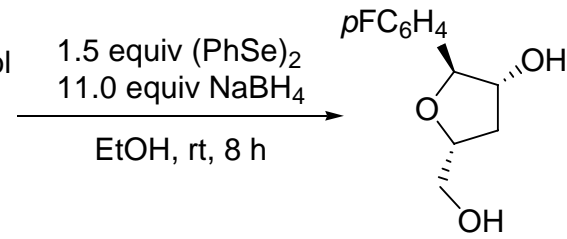

18d (60)

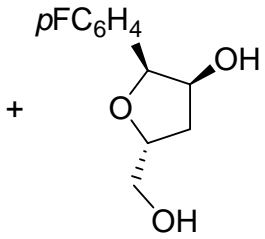

19d (40)

From a solution of epoxy sulfone $5 \mathbf{d}^{2}(55 \mathrm{mg}, 0.15 \mathrm{mmol}, 1.0$ equiv) in $0.5 \mathrm{~mL}$ of EtOH and a suspension generated from $(\mathrm{PhSe})_{2}\left(70 \mathrm{mg}, 0.22 \mathrm{mmol}, 1.5\right.$ equiv) in $1.1 \mathrm{~mL}$ of $\mathrm{EtOH}$ and $\mathrm{NaBH}_{4}(63$ $\mathrm{mg}, 1.65 \mathrm{mmol}, 11.0$ equiv), according to the general procedure (8 h), a 60:40 mixture of diols 18d and 19d was obtained. Purification by chromatography (30-80\% EtOAc- $\mathrm{CH}_{2} \mathrm{Cl}_{2}$ ) gave 18d (15 mg, 48\%) and 19d (12 mg, 36\%) as colorless oils.

Data of 18d: $\boldsymbol{R}_{\boldsymbol{f}}=0.12\left(50 \%\right.$ EtOAc- $\left.\mathrm{CH}_{2} \mathrm{Cl}_{2}\right) .{ }^{\mathbf{1}} \mathbf{H}$ NMR (200 MHz) $\delta 1.85$ (ap dt, $1 \mathrm{H}, J=13.7,2.6$ Hz), 2.26 (ddd, 1 H, $J=13.9,9.5,5.7$ Hz), 3.41 (br s, 2 H), 3.59 (dd, 1 H, $J=11.7,2.6$ Hz), 3.94 (dd, 1 $\mathrm{H}, J=11.8,2.5 \mathrm{~Hz}), 4.22$ (ap dt, $1 \mathrm{H}, J=5.7,2.0 \mathrm{~Hz}), 4.51$ (dm, $1 \mathrm{H}, J=9.5 \mathrm{~Hz}), 5.05$ (br s, $1 \mathrm{H}), 7.00$ (ap t, $2 \mathrm{H}, J=8.7 \mathrm{~Hz}), 7.24(\mathrm{~m}, 2 \mathrm{H}) .{ }^{13} \mathbf{C}$ NMR (50 MHz) $\delta$ 34.7, 64.4, 77.8, 78.9, 88.3, $115.3(2 \mathrm{C}$, $\left.J_{C-F}=21.7 \mathrm{~Hz}\right), 126.5\left(2 \mathrm{C}, J_{C-F}=8.0 \mathrm{~Hz}\right), 136.4,162.0$. IR (film): 3367, 2928, 1605, 1509, 1223, 1157, 1024, $832 \mathrm{~cm}^{-1}$. MS (ES): $235[\mathrm{M}+\mathrm{Na}]^{+}(100 \%), 213[\mathrm{M}+1]^{+}$. Anal. calcd for $\mathrm{C}_{11} \mathrm{H}_{13} \mathrm{FO}_{3}: \mathrm{C}_{\text {, }}$ 62.26; H, 6.17. Found: C, 61.90; H, 6.39.

Data of 19d: $\boldsymbol{R}_{\boldsymbol{f}}=0.08\left(50 \%\right.$ EtOAc- $\left.\mathrm{CH}_{2} \mathrm{Cl}_{2}\right) .{ }^{\mathbf{1}} \mathbf{H}$ NMR (200 MHz) $\delta 1.67(\mathrm{br}, 2 \mathrm{H}), 2.10-2.16(\mathrm{~m}$, $2 \mathrm{H}), 3.58(\mathrm{dd}, 1 \mathrm{H}, J=11.8,5.2 \mathrm{~Hz}), 3.84(\mathrm{dd}, 1 \mathrm{H}, J=12.1,2.9 \mathrm{~Hz}), 4.40(\mathrm{~m}, 1 \mathrm{H}), 4.58(\mathrm{~m}, 1 \mathrm{H})$, $5.03(\mathrm{~d}, 1 \mathrm{H}, J=2.7 \mathrm{~Hz}), 7.06($ ap t, $2 \mathrm{H}, J=8.7 \mathrm{~Hz}), 7.24(\mathrm{~m}, 2 \mathrm{H})$.

\subsection{General Procedure for Cleavage of Sulfonyl Oxiranes with $\mathrm{MgBr}_{2}$ and $\mathbf{M g I}_{2}$.}

A two-necked round-bottomed flask fitted with a condenser was charged with a suspension of dry $\mathrm{Mg}$ turnings (stored in an oven overnight) in anhydrous $\mathrm{Et}_{2} \mathrm{O}(5 \mathrm{~mL} / \mathrm{mmol}$ of $\mathrm{Mg})$. Then, 1,2-diiodoethane or 1,2-dibromoethane (1,2 equiv relative to $\mathrm{Mg}$ ) was added at $\mathrm{rt}$ and the mixture was stirred for about 30 
min or until disappearance of $\mathrm{Mg}$ was observed, to produce a ca. $0.2 \mathrm{M}$ solution of $\mathrm{MgX}_{2}$ in $\mathrm{Et}_{2} \mathrm{O}$ that could be stored in a refrigerator under argon for a few days. To a cold $\left(0^{\circ} \mathrm{C}\right)$ solution of epoxy sulfone, previously azeotroped with anhydrous cyclohexane, in anhydrous $\mathrm{Et}_{2} \mathrm{O}(5 \mathrm{~mL} / \mathrm{mmol}$ of sulfone) was added $\mathrm{MgX}_{2}$ (5-9 equiv of the above solution) and the reaction mixture was allowed to warm up to $\mathrm{rt}$ and monitored by TLC until disappearance of starting material was observed. The reaction was then quenched with $5 \% \mathrm{NaHCO}_{3}$ solution $(10 \mathrm{~mL} / \mathrm{mmol})$ and $1 \mathrm{M} \mathrm{Na}_{2} \mathrm{~S}_{2} \mathrm{O}_{4}$ solution $(10 \mathrm{~mL} / \mathrm{mmol})$, the layers were separated, the aqueous layer was extracted with EtOAc (3 x $10 \mathrm{~mL} / \mathrm{mmol})$, and the combined organic layers were washed with a saturated solution of $\mathrm{NaCl}$ and dried over anhydrous $\mathrm{MgSO}_{4}$. Removal of the drying agent and concentration of the solution under reduced pressure gave a crude product that was purified by column chromatography on silica gel using the appropriate mixture of eluents.

\subsection{Synthesis of ( \pm )-(2S,4R,5S)-4-Bromo-5-(hydroxymethyl)-2-phenyl-3-oxotetrahydrofuran, 20.}
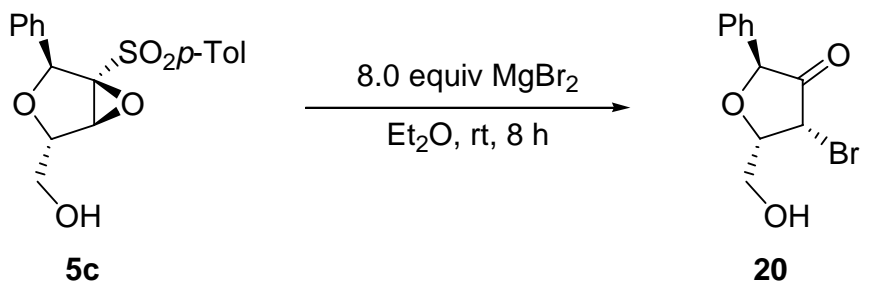

From sulfonyl oxirane $5 \mathrm{c}\left(18 \mathrm{mg}, 0.05 \mathrm{mmol}, 1.0\right.$ equiv), in $0.5 \mathrm{~mL}$ of $\mathrm{Et}_{2} \mathrm{O}$, with $\mathrm{MgBr}_{2}(8.0$ equiv in $1 \mathrm{~mL}$ of $\mathrm{Et}_{2} \mathrm{O}$ ) according to the general procedure ( $\left.\mathrm{rt}, 8 \mathrm{~h}\right)$, and after chromatography (5-40\% EtOAchexane), ketone 20 (5.0 mg, 51\%) was obtained as a colorless oil.

Data for 20: $\boldsymbol{R}_{\boldsymbol{f}}=0.52\left(50 \%\right.$ EtOAc-hexane). ${ }^{1} \mathbf{H}$ NMR (300 MHz) $\delta 3.90(\mathrm{dd}, 1 \mathrm{H}, J=12.3,3.1$ Hz), $4.11(\mathrm{dd}, 1 \mathrm{H}, J=12.5,2.6 \mathrm{~Hz}), 4.47$ (ddd, $1 \mathrm{H}, J=7.7,3.1,2.6 \mathrm{~Hz}), 4.64(\mathrm{dd}, 1 \mathrm{H}, J=7.7,1.2$ Hz), 5.25 (br s, 1 H), 7.29-7.45 (m, 5 H). IR (film): 2924, 1715 (strong), 1451, 1272, $711 \mathrm{~cm}^{-1}$. Partial ${ }^{13}$ C RMN (50 MHz) data $\delta$ 42.7, 61.4, 80.5, 83.0, 125.8, 128.5, 128.8, 133.6.

\subsection{Synthesis of (2R,5S)-5-(hydroxymethyl)-2-isopropyl-3-oxotetrahydrofuran, 15a.}

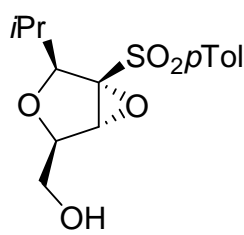

$4 a$

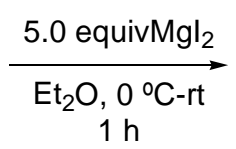

$1 \mathrm{~h}$

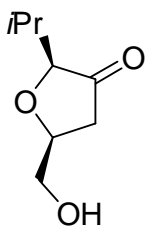

$15 a$

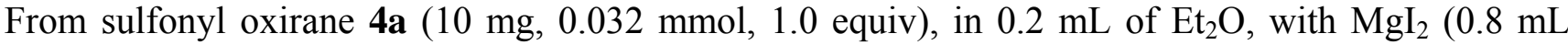
from a ca. $0.2 \mathrm{M}$ solution in $\mathrm{Et}_{2} \mathrm{O}, 5.0$ equiv) according to the general procedure $\left(0{ }^{\circ} \mathrm{C}\right.$ to $\left.\mathrm{rt}, 1 \mathrm{~h}\right)$, and after chromatography (25-50\% EtOAc-hexane), ketone 15a (5.0 mg, 97\%) was obtained. 
Data for 15a: $\quad \boldsymbol{R}_{\boldsymbol{f}}=0.25\left(70 \%\right.$ EtOAc-hexane). ${ }^{1} \mathbf{H}$ NMR (300 MHz) $\delta \quad 0.90(\mathrm{~d}, 3 \mathrm{H}, J=7.0 \mathrm{~Hz})$, $1.03(\mathrm{~d}, 3 \mathrm{H}, J=6.7 \mathrm{~Hz}), 1.50-1.62$ (br s, $1 \mathrm{H}), 2.02-2.15(\mathrm{~m}, 1 \mathrm{H}), 2.40$ (d, $1 \mathrm{H}, J=1.1 \mathrm{~Hz}), 2.43$ (d, 1 $\mathrm{H}, J=3.4 \mathrm{~Hz}), 3.67(\mathrm{dd}, 2 \mathrm{H}, J=10.6,4.1 \mathrm{~Hz}), 3.97(\mathrm{dd}, 1 \mathrm{H}, J=12.1,2.7 \mathrm{~Hz}), 4.23(\mathrm{~m}, 1 \mathrm{H}) .{ }^{13} \mathrm{C}$ NMR (75 MHz) $\delta 16.9,18.9,30.2,39.2,63.7,85.7,215.1$.

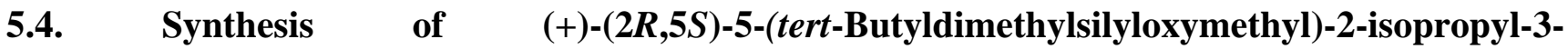
oxotetrahydrofuran, $(+)-21 a$.

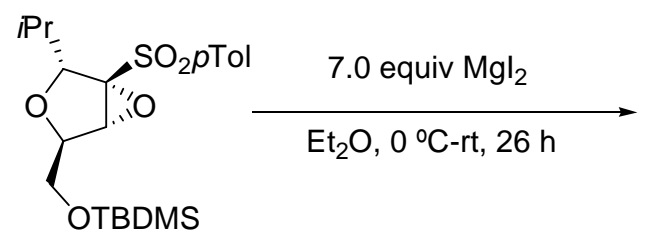

$(+)-10 a$

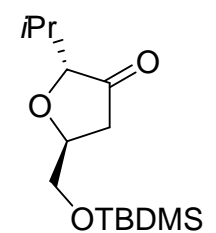

$(+)-21 a$

From sulfonyl oxirane (+)-10a (12 mg, $0.028 \mathrm{mmol}, 1.0$ equiv), in $0.2 \mathrm{~mL}$ of $\mathrm{Et}_{2} \mathrm{O}$, with $\mathrm{MgI}_{2}(0.98$ $\mathrm{mL}$ from a ca. $0.2 \mathrm{M}$ solution in $\mathrm{Et}_{2} \mathrm{O}, 7.0$ equiv) according to the general procedure $\left(0{ }^{\circ} \mathrm{C}\right.$ to $\left.\mathrm{rt}, 26 \mathrm{~h}\right)$, and after chromatography (5-30\% $\mathrm{CH}_{2} \mathrm{Cl}_{2}$-hexane), ketone (+)-21a (6.6 mg, 86\%) was obtained.

Data for (+)-21a: $\boldsymbol{R}_{\boldsymbol{f}}=0.30\left(\mathrm{CH}_{2} \mathrm{Cl}_{2}\right) .[\alpha]^{\mathbf{2 0}}{ }_{\mathbf{D}}=+55.9(c=0.51) .{ }^{1} \mathbf{H} \mathbf{N M R}(\mathbf{3 0 0} \mathbf{~ M H z}) \delta 0.025(\mathrm{~s}, 3$ H), $0.029(\mathrm{~s}, 3 \mathrm{H}), 0.85(\mathrm{~s}, 9 \mathrm{H}), 0.88(\mathrm{~d}, 3 \mathrm{H}, J=6.8 \mathrm{~Hz}), 0.99(\mathrm{~d}, 3 \mathrm{H}, J=6.9 \mathrm{~Hz}), 1.96$ (ap quint d, 1 H, $J=6.9,4.3 \mathrm{~Hz}), 2.44(\mathrm{~d}, 1 \mathrm{H}, J=1.4 \mathrm{~Hz}), 2.46$ (d, $1 \mathrm{H}, J=3.8 \mathrm{~Hz}), 3.63(\mathrm{dd}, 1 \mathrm{H}, J=10.8,2.9 \mathrm{~Hz})$, $3.83(\mathrm{~s}, 1 \mathrm{H}), 3.85(\mathrm{dd}, 1 \mathrm{H}, J=10.8,3.3 \mathrm{~Hz}), 4.43(\mathrm{td}, 1 \mathrm{H}, J=7.5,3.0 \mathrm{~Hz}) .{ }^{13} \mathbf{C} \mathbf{N M R}(75 \mathrm{MHz}) \delta$ $-5.53,-5.52,16.8,18.2,18.8,25.8$ (3 C), 31.0, 39.2, 66.8, 75.7, 84.4, 218.5. IR (film): 3398, 3014, 2956, 2928, 2855, 1754, 1464, $1215 \mathrm{~cm}^{-1}$. MS (ES): $295[\mathrm{M}+\mathrm{Na}]^{+}, 273[\mathrm{M}+1]^{+}(100 \%)$. Anal. calcd for $\mathrm{C}_{14} \mathrm{H}_{28} \mathrm{O}_{3} \mathrm{Si}: \mathrm{C}, 61.72 ; \mathrm{H}, 10.36$. Found: $\mathrm{C}, 61.89 ; \mathrm{H}, 10.03$.

\subsection{Synthesis of}

(+)-(2R,5R)-5-(tert-Butyldimethylsilyloxymethyl)-2-isopropyl-3oxotetrahydrofuran, $(+)-22 a$.

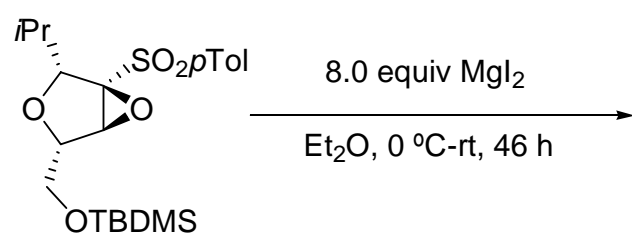

$(+)-11 a$

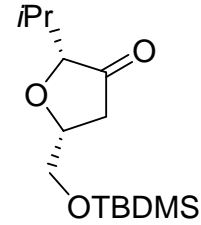

$(+)-22 a$

From sulfonyl oxirane (+)-11a (31 mg, $0.073 \mathrm{mmol}, 1.0$ equiv), in $0.4 \mathrm{~mL}$ of $\mathrm{Et}_{2} \mathrm{O}$, with $\mathrm{MgI}_{2}(2.9 \mathrm{~mL}$ from a ca. $0.2 \mathrm{M}$ solution in $\mathrm{Et}_{2} \mathrm{O}, 8.0$ equiv) according to the general procedure $\left(0{ }^{\circ} \mathrm{C}\right.$ to $\left.\mathrm{rt}, 46 \mathrm{~h}\right)$, and after chromatography (10-200\% $\mathrm{CH}_{2} \mathrm{Cl}_{2}$-hexane), ketone (+)-22a (16.3 mg, 82\%) was obtained.

Data for (+)-22a: $\quad \boldsymbol{R}_{\boldsymbol{f}}=0.31\left(\mathrm{CH}_{2} \mathrm{Cl}_{2}\right) . \quad[\alpha]^{20}{ }_{\mathbf{D}}=+64.4(c=0.64) .{ }^{1} \mathbf{H} \mathbf{~ N M R}(\mathbf{3 0 0} \mathbf{~ M H z}) \delta 0.07(\mathrm{~s}, 6$ H), $0.88(\mathrm{~s}, 9 \mathrm{H}), 0.89$ (d, $3 \mathrm{H}, J=6.1 \mathrm{~Hz}), 1.01$ (d, $3 \mathrm{H}, J=7.0 \mathrm{~Hz}), 2.03$ (hept d, $1 \mathrm{H}, J=6.9,3.9 \mathrm{~Hz}$ ), 
2.39 (s, $1 \mathrm{H}), 2.41$ (d, $1 \mathrm{H}, J=2.3 \mathrm{~Hz}), 3.64$ (d, $1 \mathrm{H}, J=3.9 \mathrm{~Hz}), 3.77$ (dd, $1 \mathrm{H}, J=11.1,4.0 \mathrm{~Hz}), 3.85$ $(\mathrm{dd}, 1 \mathrm{H}, J=11.1,3.8 \mathrm{~Hz}), 4.14-4.22(\mathrm{~m}, 1 \mathrm{H}, J=7.4,3.8 \mathrm{~Hz}) .{ }^{13} \mathbf{C}$ NMR (75 MHz) $\delta-5.4,-5.3$, 16.9, 18.4, 18.8, 25.9 (3 C), 30.3, 40.0, 64.4, 75.8, 85.6, 216.4. IR (film): 2926, 2855, 1741, 1710, 1436, 1269, $1162 \mathrm{~cm}^{-1}$. MS (ES): $295[\mathrm{M}+\mathrm{Na}]^{+}(100 \%), 273[\mathrm{M}+1]^{+}$. Anal. calcd for $\mathrm{C}_{14} \mathrm{H}_{28} \mathrm{O}_{3} \mathrm{Si}: \mathrm{C}$, 61.72; H, 10.36. Found: C, 61.37; H, 10.53.

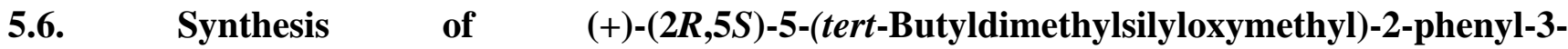
oxotetrahydrofuran, $(+)-21 c$.

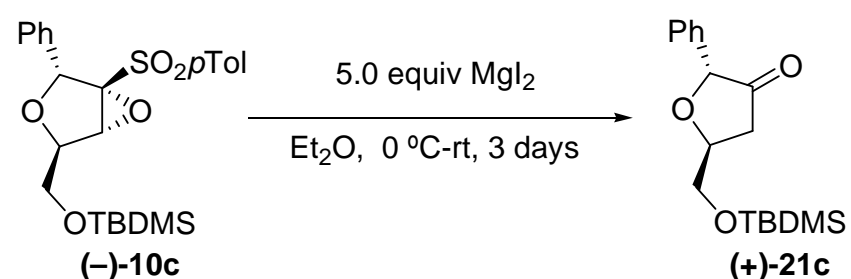

From sulfonyl oxirane (-)-10c (13 mg, $0.028 \mathrm{mmol}, 1.0$ equiv), in $0.2 \mathrm{~mL}$ of $\mathrm{Et}_{2} \mathrm{O}$, with $\mathrm{MgI}_{2}(0.7 \mathrm{~mL}$ from a ca. $0.2 \mathrm{M}$ solution in $\mathrm{Et}_{2} \mathrm{O}, 5.0$ equiv) according to the general procedure $\left(0{ }^{\circ} \mathrm{C}\right.$ to $\mathrm{rt}, 3$ days $)$, and after chromatography (2-6\% EtOAc-hexane), ketone (+)-21c (7 mg, 82\%) was obtained.

Data for $(+)-21 c: \quad R_{f}=0.25\left(10 \%\right.$ EtOAc-hexane). $[\alpha]^{20}{ }_{\mathbf{D}}=+17.6(c=0.58) .{ }^{1} \mathbf{H} \mathbf{~ N M R}(300 \mathrm{MHz})$ $\delta 0.07(\mathrm{~s}, 3 \mathrm{H}), 0.08(\mathrm{~s}, 3 \mathrm{H}), 0.89(\mathrm{~s}, 9 \mathrm{H}), 2.54(\mathrm{dd}, 1 \mathrm{H}, J=17.8,4.3 \mathrm{~Hz}), 2.65(\mathrm{dd}, 1 \mathrm{H}, J=17.8,8.1$ Hz), $3.74(\mathrm{dd}, 1 \mathrm{H}, J=11.0,2.7 \mathrm{~Hz}), 4.02(\mathrm{dd}, 1 \mathrm{H}, J=11.0,2.9 \mathrm{~Hz}), 4.67$ (dd, $1 \mathrm{H}, J=8.1,4.2 \mathrm{~Hz})$, 5.02 (s, $1 \mathrm{H}), 7.26-7.40$ (m, 5 H). ${ }^{13} \mathrm{C}$ NMR (75 MHz) $\delta-5.6,-5.5,18.2,25.8$ (3 C), 37.6, 66.7, 76.0, 81.5, 125.6 (2 C), 128.0, 128.5 (2 C), 136.9, 212.5. IR (film): 3057, 3028, 2949, 2929, 2857, 1762, 1494, 1471, 1406, 1255, $1102 \mathrm{~cm}^{-1}$. MS (ES): $329[\mathrm{M}+\mathrm{Na}]^{+}, 307[\mathrm{M}+1]^{+}$. Anal. calcd for $\mathrm{C}_{17} \mathrm{H}_{26} \mathrm{O}_{3} \mathrm{Si}$ : C, 66.62; H, 8.55. Found: C, 66.97; H, 8.83.

5.7. Synthesis of

(+)-(2R,5R)-5-(tert-Butyldimethylsilyloxymethyl)-2-phenyl-3oxotetrahydrofuran, $(+)-22 c$.

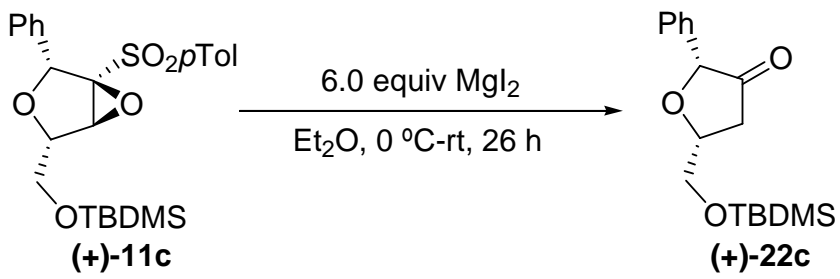

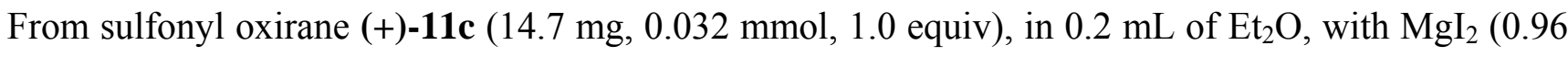
$\mathrm{mL}$ from a ca. $0.2 \mathrm{M}$ solution in $\mathrm{Et}_{2} \mathrm{O}, 6.0$ equiv) according to the general procedure $\left(0{ }^{\circ} \mathrm{C}\right.$ to $\left.\mathrm{rt}, 26 \mathrm{~h}\right)$, and after chromatography (2-6\% EtOAc-hexane), ketone (+)-22c (10 mg, 100\%) was obtained. 


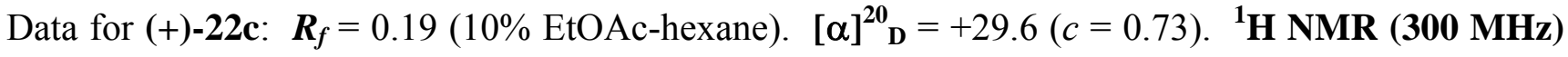
$\delta 0.09$ (s, $3 \mathrm{H}), 0.10$ (s, $3 \mathrm{H}), 0.91$ (s, 9 H), 2.52 (dd, $1 \mathrm{H}, J=17.8,5.9 \mathrm{~Hz}), 2.72$ (dd, $1 \mathrm{H}, J=17.8,9.9$ $\mathrm{Hz}$ ), 3.87 (dd, $1 \mathrm{H}, J=11.1,3.5 \mathrm{~Hz}$ ), 4.02 (dd, $1 \mathrm{H}, J=11.1,3.5 \mathrm{~Hz}$ ), 4.43 (sextet, $1 \mathrm{H}, J=9.8,3.6 \mathrm{~Hz}$ ), 4.79 (s, $1 \mathrm{H}), 7.27-7.44(\mathrm{~m}, 5 \mathrm{H}) .{ }^{13} \mathrm{C}$ NMR (75 MHz) $\delta-5.5,-5.3,18.4,25.9$ (3 C), 38.0, 64.1, 76.3, 82.9, 126.2 (2 C), 128.2, 128.4 (2 C), 136.4, 212.9. IR (film): 3061, 3032, 2952, 2929, 2850, 1722, 1471, 1449, 1272, 1110, $836 \mathrm{~cm}^{-1}$. MS (ES): $375[\mathrm{M}+3 \mathrm{Na}]^{+}(100 \%), 329[\mathrm{M}+\mathrm{Na}]^{+}, 307[\mathrm{M}+1]^{+}$. Anal. calcd for $\mathrm{C}_{17} \mathrm{H}_{26} \mathrm{O}_{3} \mathrm{Si}$ : $\mathrm{C}, 66.62 ; \mathrm{H}, 8.55$. Found: $\mathrm{C}, 66.84 ; \mathrm{H}, 8.40$.

\subsection{Synthesis of (-)-(2R,3R,5S)-5-(tert-Butyldimethylsilyloxymethyl)-2-phenyl-tetrahydrofuran-} 3-ol, (-)-23c.

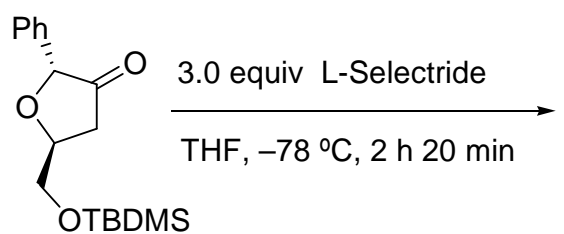

$(+)-21 c$

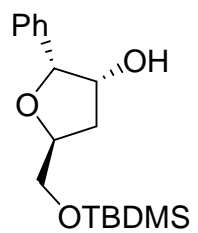

$(-)-23 c$

To a cold $\left(-78{ }^{\circ} \mathrm{C}\right)$ solution of ketone $(+)-21 \mathrm{c}(5 \mathrm{mg}, 0.016 \mathrm{mmol}, 1.0$ equiv) in $0.2 \mathrm{~mL}$ of THF was added L-Selectride $(0.048 \mathrm{~mL}, 0.048 \mathrm{mmol}, 1 \mathrm{M}$ in THF, 3.0 equiv) and the mixture was stirred at -78 ${ }^{\circ} \mathrm{C}$ for $2 \mathrm{~h}$ and $20 \mathrm{~min}$. Upon completion, the reaction was quenched with $\mathrm{MeOH}$, allowed to warm up to $\mathrm{rt}$ and the solvent was removed. Purification by chromatography (2-5\% EtOAc-hexane) gave alcohol $(-)-23 \mathrm{c}(4 \mathrm{mg}, 81 \%)$ as a colorless oil.

Data for (-)-23c: $\boldsymbol{R}_{\boldsymbol{f}}=0.43\left(10 \%\right.$ EtOAc- $\left.\mathrm{CH}_{2} \mathrm{Cl}_{2}\right) .[\alpha]^{\mathbf{2 0}}{ }_{\mathbf{D}}=-55.6(c=0.20) .{ }^{1} \mathbf{H} \mathbf{N M R}(\mathbf{3 0 0} \mathbf{~ M H z})$ $\delta 0.05$ (s, $3 \mathrm{H}), 0.06$ (s, $3 \mathrm{H}), 0.89$ (s, $9 \mathrm{H}), 2.18$ (m, $2 \mathrm{H}), 3.70$ (dd, $1 \mathrm{H}, J=10.9,3.8 \mathrm{~Hz}), 3.82$ (dd, 1 $\mathrm{H}, J=10.9,4.0 \mathrm{~Hz}), 4.42(\mathrm{br} \mathrm{s}, 1 \mathrm{H}), 4.51(\mathrm{~m}, 1 \mathrm{H}), 5.05(\mathrm{~d}, 1 \mathrm{H}, J=2.8 \mathrm{~Hz}), 7.26-7.37$ (m, $5 \mathrm{H}) . \mathbf{M S}$ (ES): $331[\mathrm{M}+\mathrm{Na}]^{+}(100 \%), 309[\mathrm{M}+1]^{+}$.

\subsection{Synthesis of (-)-(2R,3R,5R)-5-(tert-Butyldimethylsilyloxymethyl)-2-phenyl-tetrahydrofuran-} 3-ol, (-)-24c.

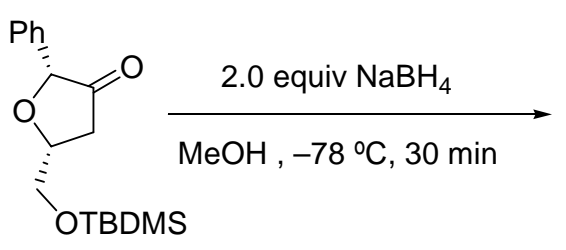

$(+)-22 c$

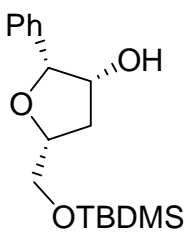

$(-)-24 c$

To a cold $\left(-78{ }^{\circ} \mathrm{C}\right)$ solution of $\mathrm{NaBH}_{4}(1.2 \mathrm{mg}, 0.031 \mathrm{mmol}, 2.0$ equiv) in $0.3 \mathrm{~mL}$ of $\mathrm{MeOH}$ was added a solution of ketone (+)-22c (4.8 mg, $0.016 \mathrm{mmol}, 1.0$ equiv) in $0.2 \mathrm{~mL}$ of $\mathrm{MeOH}$ and the mixture was stirred at $-78{ }^{\circ} \mathrm{C}$ for $30 \mathrm{~min}$. Upon completion, the reaction was quenched with saturated $\mathrm{NH}_{4} \mathrm{Cl}$ 
solution and $\mathrm{H}_{2} \mathrm{O}$ and diluted with EtOAc. Standard extractive work-up gave a crude product that was purified by chromatography (2-5\% EtOAc-hexane) to afford alcohol (-)-24c $(2.6 \mathrm{mg}, 53 \%)$ as a colorless oil.

Data for (-)-24c: $\boldsymbol{R}_{\boldsymbol{f}}=0.59\left(10 \%\right.$ EtOAc- $\left.\mathrm{CH}_{2} \mathrm{Cl}_{2}\right) .[\alpha]^{\mathbf{2 0}}{ }_{\mathbf{D}}=-50.0(c=0.19) .{ }^{\mathbf{1}} \mathbf{H} \mathbf{N M R}(\mathbf{3 0 0} \mathbf{~ M H z})$ $\delta 0.137$ (s, $3 \mathrm{H}), 0.14$ (s, $3 \mathrm{H}), 0.92$ (s, $9 \mathrm{H}), 2.08$ (dd, $1 \mathrm{H}, J=13.7,2.8 \mathrm{~Hz}$ ), 2.51 (ddd, $1 \mathrm{H}, J=13.7$, 10.0, $5.2 \mathrm{~Hz}$ ), 3.63 (dd, $1 \mathrm{H}, J=10.7,2.1 \mathrm{~Hz}), 3.69$ (d, 1H, $J=10.6 \mathrm{~Hz}), 4.00$ (dd, $1 \mathrm{H}, J=10.7,2.3$ $\mathrm{Hz}), 4.17(\mathrm{ddd}, 1 \mathrm{H}, J=10.4,5.1,3.0 \mathrm{~Hz}), 4.35(\mathrm{dq}, 1 \mathrm{H}, J=10.0,2.5 \mathrm{~Hz}), 4.82(\mathrm{~d}, 1 \mathrm{H}, J=2.6 \mathrm{~Hz})$, 7.21-7.38 (m, $5 \mathrm{H})$. MS (ES): $331[\mathrm{M}+\mathrm{Na}]^{+}(100 \%), 309[\mathrm{M}+1]^{+}$.

\subsection{Synthesis of the tert-Butyldimethylsilyl ether of $(+)-\left(2 R, 3 R, 4 R, 5 S, S_{S}\right)-3,4-E p o x y-4-(p-$} tolylsulfinyl)-5-vinyl-tetrahydrofuran-2-methanol, (+)-28a.

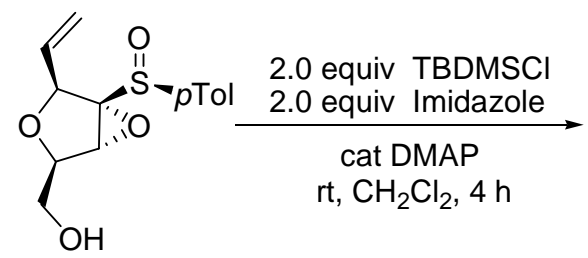

$(+)-3 f$

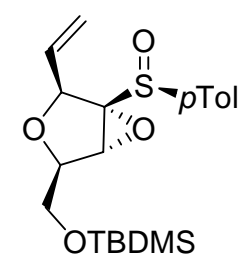

(+)-28a

From sulfinyl oxirane (+)-3f (29 mg, 0.103 mmol, 1.0 equiv), with TBDMSCl (2.0 equiv), according to the general procedure $(2 \mathrm{~h})$, silyl ether $(+)$-28a was obtained. Purification by chromatography $(5-10 \%$ EtOAc-hexane) gave (+)-28a (32 $\mathrm{mg}, 79 \%)$ as a white solid.

Data for (+)-28a: $\quad \boldsymbol{R}_{\boldsymbol{f}}=0.08$ (10\% EtOAc-hexane). $[\alpha]^{\mathbf{2 0}}{ }_{\mathbf{D}}=+74.0(c=0.10) .{ }^{1} \mathbf{H} \mathbf{N M R}(\mathbf{3 0 0} \mathbf{~ M H z})$ $\delta 0.046(\mathrm{~s}, 6 \mathrm{H}), 2.40(\mathrm{~s}, 3 \mathrm{H}), 3.71(\mathrm{dd}, 1 \mathrm{H}, J=11.0,5.4 \mathrm{~Hz}), 3.79$ (dd, $1 \mathrm{H}, J=11.0,3.7 \mathrm{~Hz}), 4.06$ (d, $1 \mathrm{H}, J=9.2 \mathrm{~Hz}$ ), 4.15 (dd, $1 \mathrm{H}, J=5.4,3.6 \mathrm{~Hz}), 4.23$ (s, $1 \mathrm{H}), 5.17$ (dm, $1 \mathrm{H}, J=16.1 \mathrm{~Hz}), 5.32$ (dm, 1 H, $J=10.1 \mathrm{~Hz}$ ), 6.08 (ddd, $1 \mathrm{H}, J=16.9,10.1,9.0 \mathrm{~Hz}$ ), 7.03 (d, $2 \mathrm{H}, J=7.9 \mathrm{~Hz}), 7.61$ (d, $2 \mathrm{H}, J=8.3$ Hz). ${ }^{13}$ C NMR (75 MHz) $\delta-5.5$ (2 C), 18.3, 21.6, 25.9 (3 C), 63.7, 65.7, 78.9, 80.0, 81.5, 121.3, 125.1, 126.4 (2 C), 129.1, 130.0 (2 C), 133.7, 136.3, 143.2. IR (KBr): 3060, 2960, 2940, 2860, 1730, 1600, 1490, 1470, 1260, 1120, 1060, 840, $780 \mathrm{~cm}^{-1}$. MS (ES): $395[\mathrm{M}+\mathrm{H}]^{+}(100 \%)$. Anal. calcd for $\mathrm{C}_{20} \mathrm{H}_{30} \mathrm{O}_{4} \mathrm{SSi}$ : C, 60.88; H, 7.66; S, 8.13. Found: C, 61.20; H, 7.41; S, 7.85.

7.2. Synthesis of the tert-Butyldiphenylsilyl ether of $(+)-\left(2 R, 3 R, 4 R, 5 S, S_{S}\right)-3,4-E p o x y-4-(p-$ tolylsulfinyl)-5-vinyl-tetrahydrofuran-2-methanol, (+)-28b. 


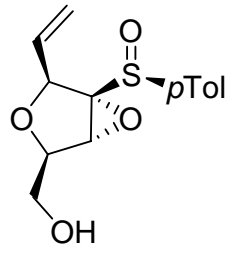

$(+)-3 f$

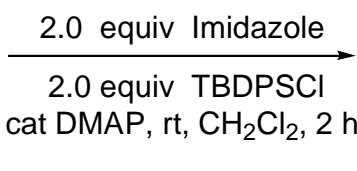

at DMP, rt, $\mathrm{CH}_{2} \mathrm{Cl}_{2}, 2$

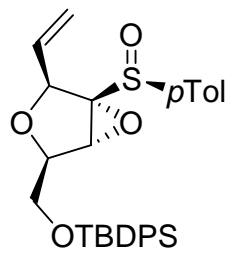

$(+)-28 b$

From sulfinyl oxirane (+)-3f (40 mg, $0.142 \mathrm{mmol}, 1$ equiv), with TBDPSCl (2.0 equiv), according to the general procedure $(2 \mathrm{~h})$, silyl ether $(+)$-28b was obtained. Purification by chromatography $(5-20 \%$ EtOAc-hexane) gave (+)-28b (60 mg, 82\%) as a white solid.

Data for $(+)-28 b: \quad R_{f}=0.23\left(20 \%\right.$ EtOAc-hexane). $[\alpha]^{\mathbf{2 0}}{ }_{\mathbf{D}}=+74.2(c=0.13) .{ }^{\mathbf{1}} \mathbf{H} \mathbf{~ N M R}(\mathbf{2 0 0} \mathbf{M H z})$ $\delta 1.10(\mathrm{~s}, 9 \mathrm{H}), 2.41(\mathrm{~s}, 3 \mathrm{H}), 3.75(\mathrm{~d}, 2 \mathrm{H}, J=4.8 \mathrm{~Hz}), 4.07(\mathrm{~d}, 1 \mathrm{H}, J=9.0 \mathrm{~Hz}), 4.18(\mathrm{t}, 1 \mathrm{H}, J=4.6$ Hz), 4.34 (s, $1 \mathrm{H}), 5.16$ (dd, $1 \mathrm{H}, J=17.0,1.0 \mathrm{~Hz}), 5.28$ (dd, $1 \mathrm{H}, J=10.2,0.8 \mathrm{~Hz}), 6.05$ (ddd, $1 \mathrm{H}, J=$

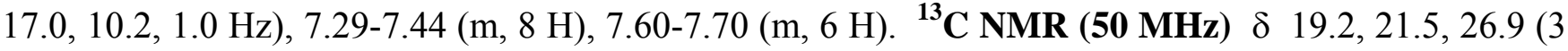
C), 64.3, 65.7, 78.8, 79.9, 81.6, 121.3, 126.3, 127.9, 127.9, 129.9, 130.0, 132.6, 132.7, 133.6, 135.5, 135.6, 136.3, 143.1. IR (KBr): 3080, 2940, 2860, 1430, 1110, 1060, 960, 940, 930, 810, 740, 700, 620 $\mathrm{cm}^{-1}$. Anal. calcd for $\mathrm{C}_{30} \mathrm{H}_{34} \mathrm{O}_{4} \mathrm{SSi}$ : C, 69.46; H, 6.61; S, 6.18. Found: C, 69.66; H, 6.42; S, 6.33.

\subsection{Synthesis of the tert-Butyldiphenylsilyl ether of $(+)-(2 R, 3 R, 4 R, 5 S)-3,4-E p o x y-4-(p-$} tolylsulfonyl)-5-vinyl-tetrahydrofuran-2-methanol, (-)-29b.

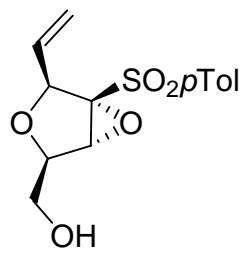

$(+)-\mathbf{4}$

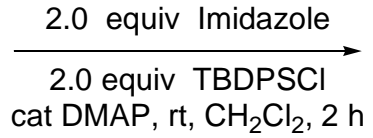
cat DMAP, rt, $\mathrm{CH}_{2} \mathrm{Cl}_{2}, 2 \mathrm{~h}$

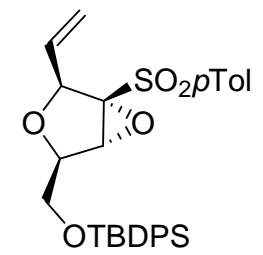

$(-)-29 b$

From sulfonyl oxirane (+)-4f (37 mg, $0.125 \mathrm{mmol}, 1.0$ equiv), with TBDPSCl (2.0 equiv), according to the general procedure $(2 \mathrm{~h})$, silyl ether (-)-29b was obtained. Purification by chromatography $(5-10 \%$ EtOAc-hexane) gave (-)-29b (56 mg, 84\%) as a white solid.

Data for (-)-29b: $\quad \boldsymbol{R}_{\boldsymbol{f}}=0.22\left(2 \times 10 \%\right.$ EtOAc-hexane). $[\alpha]^{20}{ }_{\mathbf{D}}=-11.4(c=0.37) . \quad{ }^{\mathbf{1}} \mathbf{H} \mathbf{N M R}(300$ MHz) $\delta 1.08(\mathrm{~s}, 9 \mathrm{H}), 2.45(\mathrm{~s}, 3 \mathrm{H}), 3.69$ (m, $2 \mathrm{H}), 4.11(\mathrm{dd}, 1 \mathrm{H}, J=7.1,4.8 \mathrm{~Hz}), 4.27(\mathrm{~d}, 1 \mathrm{H}, J=9.3$ $\mathrm{Hz}), 4.42(\mathrm{~s}, 1 \mathrm{H}), 5.16(\mathrm{~d}, 1 \mathrm{H}, J=16.7 \mathrm{~Hz}), 5.25(\mathrm{~d}, 1 \mathrm{H}, J=9.9 \mathrm{~Hz}), 6.12(\mathrm{dt}, 1 \mathrm{H}, J=16.8,9.5 \mathrm{~Hz})$, 7.33-7.43 (m, 8 H), 7.63-7.77 (m, 6 H). ${ }^{13}$ C NMR (75 MHz) $\delta$ 19.1, 21.8, 26.8 (3 C), 63.9, 65.9, 77.2, 77.8, 79.8, 120.0, 127.9 (2 C), 129.1 (2 C), 129.90 (2 C), 129.95, 130.01, 132.5, 132.6, 133.7, 135.5, 135.6, 135.7, 145.9. IR $\left(\mathrm{CCl}_{4}\right): 3060,2940,2860,1600,1430,1335,1110,930,790,700,660 \mathrm{~cm}^{-1}$. Anal. calcd for $\mathrm{C}_{30} \mathrm{H}_{34} \mathrm{O}_{5} \mathrm{SSi}: \mathrm{C}, 67.38 ; \mathrm{H}, 6.41 ; \mathrm{S}, 6.00$. Found: $\mathrm{C}, 67.73 ; \mathrm{H}, 6.23 ; \mathrm{S}, 6.29$. 


\subsection{General Procedure for Benzylation of Sulfinyl and Sulfonyl Tetrahydrofuran Methanols.}

To a cold $\left(0^{\circ} \mathrm{C}\right)$ suspension of 2 equiv of $\mathrm{KH}$ in THF $(10 \mathrm{~mL} / \mathrm{mmol})$ was added slowly a solution of the substrate in THF $(5 \mathrm{~mL} / \mathrm{mmol})$. After $10 \mathrm{~min}, 2$ equiv of benzyl bromide (stored over anhydrous $\mathrm{K}_{2} \mathrm{CO}_{3}$ ) was added and the mixture was allowed to warm up to $\mathrm{rt}$ and monitored by TLC. In some cases an excess of $\mathrm{KH}$ and benzyl bromide was added, as well as a catalytic amount of tetra- $n$ butylammonium iodide. Upon completion, the reaction was quenched with saturated $\mathrm{NH}_{4} \mathrm{Cl}$ solution (5 $\mathrm{mL} / \mathrm{mmol})$, diluted with EtOAc $(5 \mathrm{~mL} / \mathrm{mmol})$, the layers were separated and the aqueous layer was extracted with EtOAc (3 times, $10 \mathrm{~mL} / \mathrm{mmol}$ ). The combined organic extracts were washed with saturated $\mathrm{NaCl}$ solution $(10 \mathrm{~mL} / \mathrm{mmol})$, dried over $\mathrm{MgSO}_{4}$ and concentrated to yield a crude product that was purified by chromatography with the appropriate mixture of eluents.

\subsection{Synthesis of the benzyl ethers of $(+)-\left(2 R, 3 R, 4 R, 5 S, S_{S}\right)-3,4-E p o x y-4-(p$-tolylsulfinyl)-5-vinyl-} tetrahydrofuran-2-methanol, 28c, and of $(+)-(2 R, 3 R, 4 R, 5 S)-3,4-E p 0 x y-4-(p$-tolylsulfonyl)-5-vinyltetrahydrofuran-2-methanol, 29c.

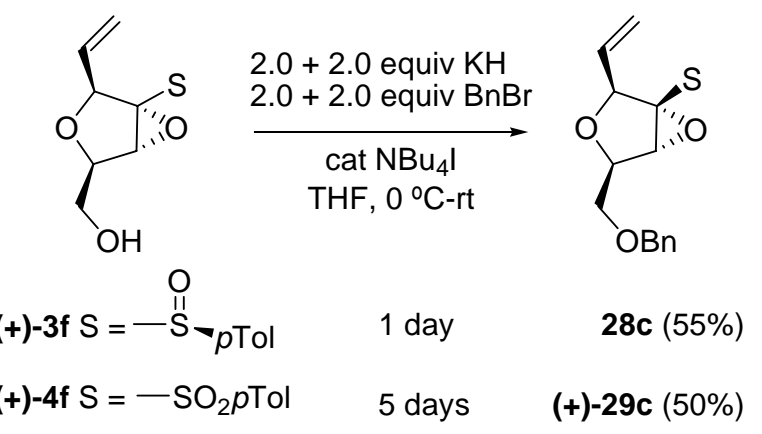

From sulfinyl alcohol (+)-3f (25mg, $0.089 \mathrm{mmol}, 1.0$ equiv), with KH (14.2 mg, $0.356 \mathrm{mmol}, 4.0$ equiv), $\mathrm{BnBr}\left(42.2 \mu \mathrm{L}, 0.356 \mathrm{mmol}, 2.0\right.$ equiv) and a catalytic amount of $\mathrm{Bu}_{4} \mathrm{NI}$ in $0.45 \mathrm{~mL}$ of $\mathrm{THF}$, following the general procedure (1 day), benzyl ether 28c (18 mg, $0.049 \mathrm{mmol}, 55 \%)$ was isolated as a colorless oil after chromatography (10-50\% EtOAc-hexane).

In a related experiment, from sulfonyl oxirane (+)-4f, after 5 days, benzyl ether $(+)-29 c(50 \%)$ was obtained as a colorless oil.

Partial data for 28c: $\boldsymbol{R}_{\boldsymbol{f}}=0.29$ (50\% EtOAc-hexane). ${ }^{1} \mathbf{H}$ NMR (300 MHz) $\delta 2.38(\mathrm{~s}, 3 \mathrm{H}), 3.59$ (s, $1 \mathrm{H}), 3.61$ (s, $1 \mathrm{H}), 3.94$ (d, $1 \mathrm{H}, J=12.5 \mathrm{~Hz}), 4.07$ (d, $1 \mathrm{H}, J=12.4 \mathrm{~Hz}), 4.26$ (t, $1 \mathrm{H}, J=3.3 \mathrm{~Hz}), 4.54$ $(\mathrm{d}, 1 \mathrm{H}, J=12.2 \mathrm{~Hz}), 4.60(\mathrm{~d}, 1 \mathrm{H}, J=12.2 \mathrm{~Hz}), 5.17(\mathrm{ddd}, 1 \mathrm{H}, J=16.9,1.5,1.0 \mathrm{~Hz}), 5.29(\mathrm{dq}, 1 \mathrm{H}, J$ $=10.3,0.7 \mathrm{~Hz}), 6.07(\mathrm{ddd}, 1 \mathrm{H}, J=16.9,10.2,9.2 \mathrm{~Hz}), 6.98(\mathrm{~d}, 2 \mathrm{H}, J=8.3 \mathrm{~Hz}), 7.21-7.37$ (m, $5 \mathrm{H})$, $7.60(\mathrm{~d}, 2 \mathrm{H}, J=8.3 \mathrm{~Hz}) .{ }^{13} \mathrm{C}$ NMR (75 MHz) $\delta 21.5,63.8,65.5,70.2,73.7,80.1,81.5,121.4,124.5$, 126.4 (2 C), 127.7 (2 C), 128.5 (2 C), 129.5, 129.9 (2 C), 133.6, 137.4, 143.2. IR (film): 3400, 2921, 1645, 1493, 1086, 1059, 927, 809, 738, $698 \mathrm{~cm}^{-1}$. MS (ES): $393[\mathrm{M}+\mathrm{Na}]^{+}, 371[\mathrm{M}+\mathrm{H}]^{+}(100 \%)$. Anal. calcd for $\mathrm{C}_{21} \mathrm{H}_{22} \mathrm{O}_{4} \mathrm{~S}: \mathrm{C}, 68.08 ; \mathrm{H}, 5.99 ; \mathrm{S}, 8.66$. Found: $\mathrm{C}, 68.40 ; \mathrm{H}, 6.26 ; \mathrm{S}, 8.31$. 
Data for (+)-29c: $\boldsymbol{R}_{\boldsymbol{f}}=0.17\left(20 \%\right.$ EtOAc-hexane). $[\alpha]^{\mathbf{2 0}}{ }_{\mathbf{D}}=+3.5(c=0.60) .{ }^{1} \mathbf{H}$ NMR (300 MHz) $\delta$ 2.44 (s, $3 \mathrm{H}$ ), 3.56 (ddd, $2 \mathrm{H}, J=15.0,10.1,5.0 \mathrm{~Hz}), 4.20$ (dd, $1 \mathrm{H}, J=5.9,4.4 \mathrm{~Hz}), 4.27$ (d, $1 \mathrm{H}, J=$ $4.6 \mathrm{~Hz}), 4.31$ (d, $1 \mathrm{H}, J=1.7 \mathrm{~Hz}), 4.51$ (d, $1 \mathrm{H}, J=12.1 \mathrm{~Hz}), 4.60$ (d, $1 \mathrm{H}, J=12.1 \mathrm{~Hz}), 5.19$ (dt, $1 \mathrm{H}, J$ $=17.1,1.0 \mathrm{~Hz}$ ), 5.27 (dt, $1 \mathrm{H}, J=10.0,1.1 \mathrm{~Hz}), 6.18$ (ddd, $1 \mathrm{H}, J=16.8,10.0,8.8 \mathrm{~Hz}), 7.32-7.37$ (m, 7 H), $7.76(\mathrm{~d}, 2 \mathrm{H}, J=8.5 \mathrm{~Hz}) .{ }^{13} \mathrm{C}$ NMR (125 MHz) $\delta$ 21.7, 63.0, 65.7, 69.9, 73.6, 76.4, 80.0, 120.0, 127.7, 128.5 (2 C), 128.7 (2 C), 129.2 (2 C), 129.5, 129.8 (2 C), 133.8, 137.4, 145.9. IR (film): 3400, 3031, 2923, 1596, 1495, 1454, 1332, 1143, 1087, 933, 814, 738, 698, $665 \mathrm{~cm}^{-1}$. MS (ES): 409 $[\mathrm{M}+\mathrm{Na}]^{+}(100 \%), 387[\mathrm{M}+\mathrm{H}]^{+}$. Anal. calcd for $\mathrm{C}_{21} \mathrm{H}_{22} \mathrm{O}_{5} \mathrm{~S}: \mathrm{C}, 65.27 ; \mathrm{H}, 5.74 ; \mathrm{S}, 8.30$. Found: $\mathrm{C}$, $65.01 ; \mathrm{H}, 6.03 ; \mathrm{S}, 8.64$.

\subsection{Synthesis of the tert-Butyldiphenylsilyl ether of $(Z, 2 S)$-5-ethylidene-4-oxotetrahydrofuran-2-} methanol, (-)-31b.

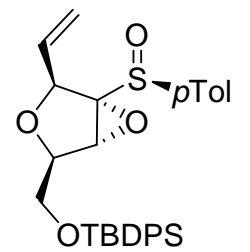

$(+)-28 b$

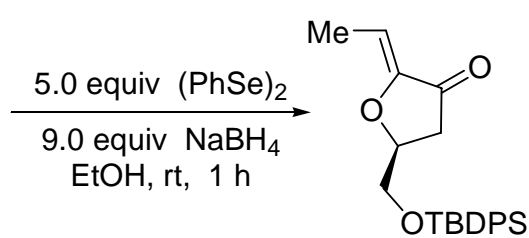

$(-)-31 b$

From a solution of epoxy sulfoxide (+)-28b $(23 \mathrm{mg}, 0.044 \mathrm{mmol}, 1.0$ equiv) in $0.3 \mathrm{~mL}$ of EtOH and a suspension generated from ( $\mathrm{PhSe})_{2}\left(68 \mathrm{mg}, 0.218 \mathrm{mmol}, 5.0\right.$ equiv) in $1.0 \mathrm{~mL}$ of EtOH and $\mathrm{NaBH}_{4}(15$ $\mathrm{mg}, 0.396 \mathrm{mmol}, 9.0$ equiv), according to the general procedure $(1 \mathrm{~h})$ but without bubbling Argon through the mixture, enone (-)-31b was obtained. Purification by chromatography (5-15\% EtOAchexane) gave (-)-31b (12 mg, 73\%) as a colorless oil. The stereochemical assignment for the exocyclic alkene is tentative.

Data for (-)-31b: $\boldsymbol{R}_{\boldsymbol{f}}=0.22\left(10 \%\right.$ EtOAc-hexane). $[\alpha]^{\mathbf{2 0}}{ }_{\mathbf{D}}=-9.2(c=0.90) .{ }^{\mathbf{1}} \mathbf{H} \mathbf{N M R}(\mathbf{3 0 0} \mathbf{~ M H z}) \delta$ 0.97 (s, $9 \mathrm{H}), 1.77$ (d, $3 \mathrm{H}, J=7.6 \mathrm{~Hz}$ ), 2.72 (d, $2 \mathrm{H}, J=6.4 \mathrm{~Hz}), 3.67$ (dd, $1 \mathrm{H}, J=11.2,2.8 \mathrm{~Hz}), 3.93$ (dd, $1 \mathrm{H}, J=2.8 \mathrm{~Hz}), 4.66$ (tt, $1 \mathrm{H}, J=6.6,2.8 \mathrm{~Hz}), 5.45$ (q, $1 \mathrm{H}, J=7.4 \mathrm{~Hz}), 7.30-7.42$ (m, $6 \mathrm{H}), 7.61-$ 7.66 (m, 4 H). ${ }^{13}$ C NMR (75 MHz) $\delta$ 10.6, 19.1, 26.5 (3 C), 37.4, 66.1, 76.6, 100.8, 127.7 (2 C), 127.8 (2 C), 129.7, 129.8, 132.8, 133.0, 135.5 (2 C), 135.6 (2 C), 149.6, 198.1. IR (film): 3436, 3065, 3014, 2956, 2929, 2857, 1736, 1660, 1472, 1428, 1390, 1260, 1113, 822, 758, 702, $666 \mathrm{~cm}^{-1}$. MS (ES): $403[\mathrm{M}+\mathrm{Na}]^{+}, 381[\mathrm{M}+\mathrm{H}]^{+}$. Anal. calcd for $\mathrm{C}_{23} \mathrm{H}_{28} \mathrm{O}_{3} \mathrm{Si}: \mathrm{C}, 72.59 ; \mathrm{H}, 7.42$. Found: $\mathrm{C}, 72.22 ; \mathrm{H}, 7.71$. 
$\mathrm{S} 30$

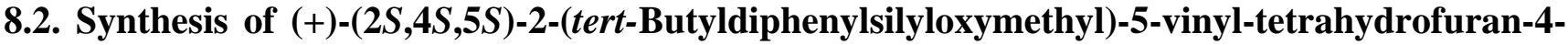
ol, (+)-26b, and (-)-(2S,4R,5S)-2-(tert-Butyldiphenylsilyloxymethyl)-5-vinyl-tetrahydrofuran-4-ol, $(-)-32 b$.
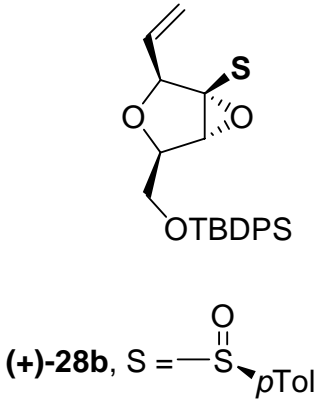

$(-)-29 b, S=-\mathrm{SO}_{2} p \mathrm{Tol}$

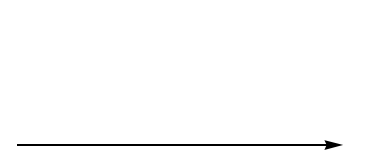

3.0 equiv $(\mathrm{PhSe})_{2}$ 26.0 equiv $\mathrm{NaBH}_{4}$ $\mathrm{EtOH},-10$ to $0^{\circ} \mathrm{C}, 3 \mathrm{~h}$ 4.0 equiv $(\mathrm{PhSe})_{2}$ 26.0 equiv $\mathrm{NaBH}_{4}$ $\mathrm{EtOH}, 0^{\circ} \mathrm{C}$ to rt, $28 \mathrm{~h}$ $90 \%$ conversion

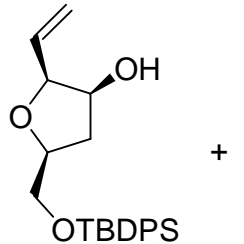

$(+)-26 b$

87

87

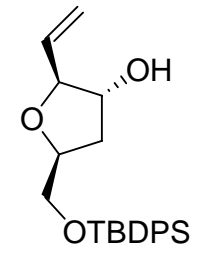

$(-)-32 b$

13

13

From a solution of epoxy sulfoxide (+)-28b $(58 \mathrm{mg}, 0.112 \mathrm{mmol}, 1.0$ equiv) in $3.6 \mathrm{~mL}$ of EtOH and a suspension generated from $(\mathrm{PhSe})_{2}\left(105 \mathrm{mg}, 0.336 \mathrm{mmol}, 3.0\right.$ equiv) in $2.7 \mathrm{~mL}$ of EtOH and $\mathrm{NaBH}_{4}$ (111 mg, $2.92 \mathrm{mmol}, 26.0$ equiv), according to the general procedure $(3 \mathrm{~h}$ ), an 87:13 mixture of alcohols (+)-26b and (-)-32b was obtained. Purification by chromatography (5-30\% EtOAc-hexane) gave (+)26b (29 mg, 68\%) and (-)-32b (4 mg, 9\%) as colorless oils.

From a solution of epoxy sulfone (-)-29b (50 mg, $0.093 \mathrm{mmol}, 1.0$ equiv) in $3 \mathrm{~mL}$ of EtOH and a suspension generated from $(\mathrm{PhSe})_{2}\left(116 \mathrm{mg}, 0.372 \mathrm{mmol}, 4.0\right.$ equiv) in $3.0 \mathrm{~mL}$ of EtOH and $\mathrm{NaBH}_{4}$ (111 mg, $2.92 \mathrm{mmol}, 26.0$ equiv), according to the general procedure $(28 \mathrm{~h})$, an 87:13 mixture of alcohols (+)-26b and (-)-32b was obtained with 90\% conversion. Purification by chromatography (530\% EtOAc-hexane) gave (+)-26b (22 mg, 61\%) and (-)-32b (3 mg, 8\%) as colorless oils.

Data for $(+)$-26b: $\quad \boldsymbol{R}_{\boldsymbol{f}}=0.34\left(30 \%\right.$ EtOAc-hexane). $[\alpha]^{\mathbf{2 0}}{ }_{\mathbf{D}}=+65.8(c=0.79) .{ }^{\mathbf{1}} \mathbf{H} \mathbf{N M R}(\mathbf{3 0 0} \mathbf{~ M H z})$ $\delta 1.06(\mathrm{~s}, 9 \mathrm{H}), 2.12(\mathrm{ddd}, 1 \mathrm{H}, J=13.8,3.2,0.5 \mathrm{~Hz}), 2.40(\mathrm{ddd}, 1 \mathrm{H}, J=13.8,10.1,5.1 \mathrm{~Hz}), 3.49$ (dd, $1 \mathrm{H}, J=11.1,2.1 \mathrm{~Hz}), 3.80$ (d, $1 \mathrm{H}, J=10.6 \mathrm{~Hz}), 3.87$ (dd, $1 \mathrm{H}, J=11.0,2.5 \mathrm{~Hz}), 4.12$ (m, $1 \mathrm{H}), 4.16-$ 4.22 (m, 2 H, H-2), 5.32 (ddd, $1 \mathrm{H}, J=10.5,1.9,1.0 \mathrm{~Hz}$ ), 5.43 (ddd, $1 \mathrm{H}, J=17.4,1.9,1.2 \mathrm{~Hz}$ ), 6.08 $(\mathrm{ddd}, 1 \mathrm{H}, J=17.3,10.5,6.5 \mathrm{~Hz}), 7.35-7.46(\mathrm{~m}, 6 \mathrm{H}), 7.67-7.76(\mathrm{~m}, 4 \mathrm{H}) .{ }^{1} \mathbf{H} \mathbf{N M R}\left(\mathbf{C}_{6} \mathbf{D}_{6}, \mathbf{3 0 0} \mathbf{M H z}\right)$ $\delta 1.16(\mathrm{~s}, 9 \mathrm{H}), 1.83(\mathrm{~m}, 2 \mathrm{H}), 3.31(\mathrm{dd}, 1 \mathrm{H}, J=10.6,2.2 \mathrm{~Hz}), 3.41(\mathrm{~d}, 1 \mathrm{H}, J=9.9 \mathrm{~Hz}), 3.77-3.93$ (m, $4 \mathrm{H}), 5.22$ (ddd, $1 \mathrm{H}, J=10.5,2.1,1.0 \mathrm{~Hz}), 5.43$ (ddd, $1 \mathrm{H}, J=17.3,2.2,1.2 \mathrm{~Hz}), 6.23$ (ddd, $1 \mathrm{H}, J=$ 17.3, 10.4, 6.1 Hz), 7.19-7.27 (m, 6 H), 7.74-7.77 (m, 2 H), 7.85-7.88 (m, 2 H). ${ }^{13}$ C NMR (75 MHz) $\delta$ 19.1, 26.7 (3 C), 37.3, 66.2, 73.3, 77.5, 85.4, 118.0, 127.8 (4 C), 129.9, 130.0, 132.2, 132.5, 134.1, 135.6 (2 C), 135.7 (2 C). IR $\left(\mathrm{CCl}_{4}\right):$ 3440, 3060, 2940, 2860, 1470, 1430, 1185, 1110, 1070, 1035, 920, $790,650,610 \mathrm{~cm}^{-1}$. Anal. calcd for $\mathrm{C}_{23} \mathrm{H}_{30} \mathrm{O}_{3} \mathrm{Si}: \mathrm{C}, 72.21 ; \mathrm{H}, 7.90$. Found: $\mathrm{C}, 72.02 ; \mathrm{H}, 8.23$. 
Data for (-)-32b: $\quad \boldsymbol{R}_{\boldsymbol{f}}=0.20$ (30\% EtOAc-hexane). $[\alpha]^{\mathbf{2 0}}{ }_{\mathbf{D}}=-7.5(c=0.28) .{ }^{\mathbf{1}} \mathbf{H} \mathbf{N M R}(300 \mathrm{MHz}) \delta$ 1.04 (s, 9 H), 1.66 (br s, $1 \mathrm{H}), 1.91$ (ddd, $1 \mathrm{H}, J=12.9,6.8,4.0 \mathrm{~Hz}), 2.12$ (ddd, $1 \mathrm{H}, J=12.9,8.1,6.0$ Hz), 3.68 (dd, $1 \mathrm{H}, J=10.7,4.1 \mathrm{~Hz}), 3.74$ (dd, $1 \mathrm{H}, J=10.8,4.3 \mathrm{~Hz}), 4.17-4.20$ (m, $2 \mathrm{H}, \mathrm{H}-4), 4.29$ (m, $1 \mathrm{H}), 5.13(\mathrm{dm}, 1 \mathrm{H}, J=10.4 \mathrm{~Hz}), 5.34(\mathrm{dm}, 1 \mathrm{H}, J=17.1 \mathrm{~Hz}), 5.83$ (ddd, $1 \mathrm{H}, J=17.0,10.4,6.3 \mathrm{~Hz}$ ), 7.35-7.45 (m, $6 \mathrm{H}), 7.64-7.69$ (m, $4 \mathrm{H}) .{ }^{\mathbf{1}} \mathbf{H}$ NMR (C $\left.\mathbf{6}_{\mathbf{6}} \mathbf{D}_{\mathbf{6}}, \mathbf{3 0 0} \mathbf{M H z}\right) \delta 0.84(\mathrm{~d}, 1 \mathrm{H}, J=6.6 \mathrm{~Hz}), 1.16$ (s, $9 \mathrm{H}), 1.57$ (ddd, $1 \mathrm{H}, J=12.8,6.4,3.4 \mathrm{~Hz}), 1.84$ (ddd, $1 \mathrm{H}, J=12.8,8.7,6.2 \mathrm{~Hz}), 3.65$ (dd, $1 \mathrm{H}, J=$ 10.8, 4.4 Hz), 3.70-3.80 (m, $1 \mathrm{H}), 3.78$ (dd, $1 \mathrm{H}, J=10.9,3.9 \mathrm{~Hz}), 4.13$ (m, $1 \mathrm{H}), 4.22-4.30$ (m, $1 \mathrm{H})$, $5.00(\mathrm{dm}, 1 \mathrm{H}, J=10.5 \mathrm{~Hz}), 5.34(\mathrm{dm}, 1 \mathrm{H}, J=17.1 \mathrm{~Hz}), 5.80(\mathrm{ddd}, 1 \mathrm{H}, J=17.2,10.4,5.9 \mathrm{~Hz}), 7.21$ $7.24(\mathrm{~m}, 6 \mathrm{H}), 7.78-7.82(\mathrm{~m}, 4 \mathrm{H})$. DNOE $\left(\mathbf{C}_{6} \mathbf{D}_{6}\right)$ between $\mathrm{CH}=\mathrm{CH}_{2} / \mathrm{CH}=\mathrm{C}_{2}(5.00 \mathrm{ppm}): 3.0 \%$; between $\mathrm{C} \underline{\mathrm{H}}=\mathrm{CH}_{2} / \mathrm{H}-5$ : $2.5 \%$; between $\mathrm{C} \underline{\mathrm{H}}=\mathrm{CH}_{2} / \mathrm{H}-4: 2.5 \%$; between $\mathrm{H}-2 / \mathrm{CH}_{2} \mathrm{OTBDPS}$ : $1.3 \%$ for each H; between H-2/H-3: 3.4\%. ${ }^{13}$ C NMR (50 MHz) $\delta 19.3,26.9$ (3 C), 36.3, 66.1, 78.1, 78.6, 87.4, 116.6, 127.7 (2 C), 129.6, 133.5, 135.7 (2 C), 137.1. MS (EI): 325, 247, 241, 223, 199 (100\%), 181, $163,139,105,91,77,57,41$. Anal. calcd for $\mathrm{C}_{23} \mathrm{H}_{30} \mathrm{O}_{3} \mathrm{Si}: \mathrm{C}, 72.21$; H, 7.90. Found: C, 71.93; H, 8.05.

\subsection{Synthesis of $(2 S, 4 S, 5 S)-4-H y d r o x y-5-v i n y l-t e t r a h y d r o f u r a n-2-m e t h a n o l,(+)-26 d$, and} $(2 S, 4 R, 5 S)-4-H y d r o x y-5$-vinyl-tetrahydrofuran-2-methanol, (-)-32d.

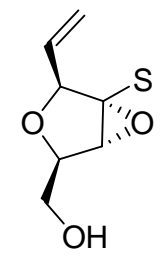

(+)-3f, $S=\stackrel{\mathrm{O}}{\text { II }}$ S $_{-p \text { Tol }}$

$(+)-4 f, S=-\mathrm{SO}_{2} p$ Tol

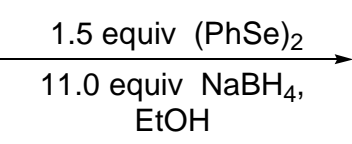

$0^{\circ} \mathrm{C}, 4 \mathrm{~h}$

$\mathrm{rt}, 19 \mathrm{~h}$

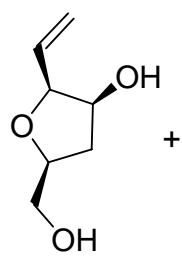

(+)-26d 76 76

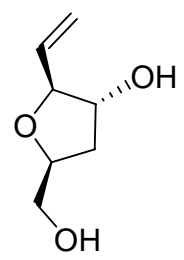

$(-)-32 d$

24

24

From a solution of epoxy sulfoxide (+)-3f (56 mg, $0.20 \mathrm{mmol}, 1.0$ equiv) in $0.6 \mathrm{~mL}$ of EtOH and a suspension generated from $(\mathrm{PhSe})_{2}\left(94 \mathrm{mg}, 0.30 \mathrm{mmol}, 1.5\right.$ equiv) in $1.5 \mathrm{~mL}$ of $\mathrm{EtOH}$ and $\mathrm{NaBH}_{4}(83$ $\mathrm{mg}, 2.20 \mathrm{mmol}, 11.0$ equiv), according to the general procedure $(4 \mathrm{~h})$, a 76:24 mixture of alcohols $(+)-$ 26d and (-)-32d (20 mg, 69\%) was obtained after chromatography (30-100\% EtOAc-hexane). These diols could be separated by a second more careful chromatography.

In a related experiment under similar conditions, from epoxy sulfone (+)-4f $(59 \mathrm{mg}, 0.2 \mathrm{mmol})$, a 76:24 mixture of alcohols (+)-26d and (-)-32d (17 mg, 60\%) was obtained.

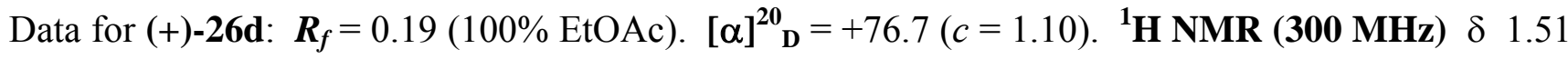
(br s, $1 \mathrm{H}), 1.96(\mathrm{dd}, 1 \mathrm{H}, J=13.8 \mathrm{~Hz}, 2.5 \mathrm{~Hz}), 2.37$ (dd, $1 \mathrm{H}, J=13.8,3.5 \mathrm{~Hz}), 2.42$ (dd, $1 \mathrm{H}, J=13.8$, $3.5 \mathrm{~Hz}), 3.56(\mathrm{dd}, 1 \mathrm{H}, J=11.6,2.7 \mathrm{~Hz}), 3.88(\mathrm{dd}, 1 \mathrm{H}, J=11.6,2.4 \mathrm{~Hz}), 4.11-4.38$ (m, $2 \mathrm{H}), 5.33$ (dm, $1 \mathrm{H}, J=10.6 \mathrm{~Hz}), 5.41(\mathrm{dm}, 1 \mathrm{H}, J=17.1 \mathrm{~Hz}), 5.99(\mathrm{ddd}, 1 \mathrm{H}, J=17.0,10.6 .6 .3 \mathrm{~Hz}) .{ }^{13} \mathbf{C} \mathbf{N M R}(\mathbf{5 0}$ 
MHz) $\delta 37.1,64.4,72.9,77.2,85.1,118.7,133.5 . \quad$ IR (film): 3368, 2929, 1645, 1444, 1286, 1186, 1054, 993, 932, 874, $814 \mathrm{~cm}^{-1}$. MS (ES): $167[\mathrm{M}+\mathrm{Na}]^{+}(100 \%)$. Anal. calcd for $\mathrm{C}_{7} \mathrm{H}_{12} \mathrm{O}_{3}: \mathrm{C}, 58.32 ; \mathrm{H}$, 8.39. Found: C, 58.60; H, 8.22.

Data for (-)-32d: $\boldsymbol{R}_{\boldsymbol{f}}=0.17\left(100 \%\right.$ EtOAc). $[\alpha]^{\mathbf{2 0}}{ }_{\mathbf{D}}=-16.5(c=0.60) .{ }^{\mathbf{1}} \mathbf{H} \mathbf{N M R}(\mathbf{2 0 0} \mathbf{~ M H z}) \delta 1.86$ (ddd, $1 \mathrm{H}, J=13.1,6.6,3.4 \mathrm{~Hz}), 2.00(\mathrm{ddd}, 1 \mathrm{H}, J=13.0,8.8,6.0 \mathrm{~Hz}), 3.52(\mathrm{dd}, 1 \mathrm{H}, J=11.8,5.0 \mathrm{~Hz}$ ), 3.78 (dd, $1 \mathrm{H}, J=11.8,3.0 \mathrm{~Hz}$ ), 4.11-4.36 (m, $3 \mathrm{H}, \mathrm{H}-2, \mathrm{H}-4), 5.17$ (dt, $1 \mathrm{H}, J=10.3,1.3 \mathrm{~Hz}), 5.32$ (dt, $1 \mathrm{H}, J=17.1,1.3 \mathrm{~Hz}), 5.80(\mathrm{ddd}, 1 \mathrm{H}, J=17.0,10.3,6.4 \mathrm{~Hz}) .{ }^{13} \mathbf{C} \mathbf{N M R}(\mathbf{5 0} \mathbf{M H z}) \delta 35.6,64.6,76.5$, 78.7, 87.5, 117.1, 136.6. IR (film): 3368, 2927, 1645, 1427, 1041, $928 \mathrm{~cm}^{-1}$. MS (ES): $167[\mathrm{M}+\mathrm{Na}]^{+}$ (100\%). Anal. calcd for $\mathrm{C}_{7} \mathrm{H}_{12} \mathrm{O}_{3}: \mathrm{C}, 58.32 ; \mathrm{H}, 8.39$. Found: $\mathrm{C}, 58.49 ; \mathrm{H}, 8.64$.

\subsection{Synthesis of $(+)-(2 S, 4 S, 5 S)-2-(B e n z y l o x y m e t h y l)-5-v i n y l-t e t r a h y d r o f u r a n-4-o l, ~(+)-26 c$, and} (2S,4R,5S)-2-(Benzyloxymethyl)-5-vinyl-tetrahydrofuran-4-ol, 32c.
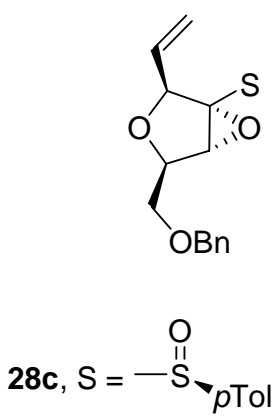

$(+)-29 c, S=-\mathrm{SO}_{2} p \mathrm{Tol}$

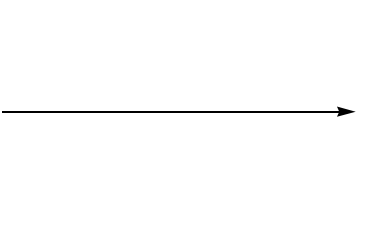

3.0 equiv (PhSe) 2 16.0 equiv $\mathrm{NaBH}_{4}$ 1.0 equiv $\mathrm{AcOH}$ $\mathrm{EtOH}, 0^{\circ} \mathrm{C}, 2 \mathrm{~h}$ 4.5 equiv $(\mathrm{PhSe})_{2}$ 30.0 equiv $\mathrm{NaBH}_{4}$ 3.0 equiv $\mathrm{AcOH}$ $\mathrm{EtOH}, 0^{\circ} \mathrm{C}$ to rt, 4 days

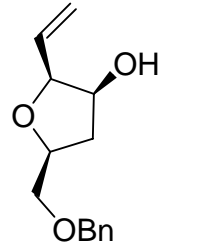

$(+)-26 c$

76

76

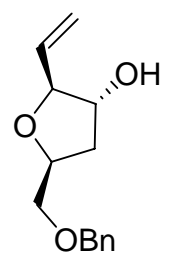

32c

24

24

From a solution of epoxy sulfoxide 28c (18 mg, $0.049 \mathrm{mmol}, 1.0$ equiv) in $0.1 \mathrm{~mL}$ of EtOH and a suspension generated from $(\mathrm{PhSe})_{2}\left(45.9 \mathrm{mg}, 0.147 \mathrm{mmol}, 3.0\right.$ equiv) in $0.4 \mathrm{~mL}$ of EtOH and $\mathrm{NaBH}_{4}$ (29.6 mg, $0.784 \mathrm{mmol}, 16.0$ equiv), and with $\mathrm{AcOH}(3 \mu \mathrm{L}, 0.049 \mathrm{mmol}, 1.0$ equiv) according to the general procedure $(2 \mathrm{~h})$, a 76:24 mixture of alcohols (+)-26c and 32c $(8 \mathrm{mg}, 70 \%)$ was obtained. Purification by chromatography $\left(0-10 \%\right.$ EtOAc- $\left.\mathrm{CH}_{2} \mathrm{Cl}_{2}\right)$ gave (+)-26c $(6 \mathrm{mg}, 53 \%)$. Minor product 32c could not be isolated.

In a related experiment, from a solution of epoxy sulfone (+)-29c (15 $\mathrm{mg}, 0.039 \mathrm{mmol}, 1.0$ equiv) and a suspension generated from $(\mathrm{PhSe})_{2}(54.8 \mathrm{mg}, 0.176 \mathrm{mmol}, 4.5 \mathrm{equiv})$ and $\mathrm{NaBH}_{4}(44.3 \mathrm{mg}, 1.17$ mmol, 30 equiv), and with $\mathrm{AcOH}(7 \mu \mathrm{L}, 0.117 \mathrm{mmol}, 3.0$ equiv) according to the general procedure (4 days), a 38:12:50 mixture of alcohols (+)-26c and 32c and (+)-29c was obtained. Purification by chromatography (0-20\% EtOAc-hexane) gave (+)-29c (7 mg, 47\%) and (+)-26c $(2.7 \mathrm{mg}, 30 \%)$. Minor product 32c could not be isolated. 
Data for (+)-26c: $\boldsymbol{R}_{\boldsymbol{f}}=0.11\left(40 \%\right.$ EtOAc-hexane). $[\alpha]^{\mathbf{2 0}}{ }_{\mathbf{D}}=+3.0(c=0.40) .{ }^{1} \mathbf{H}$ NMR (400 MHz) $\delta$ 1.98 (dd, $1 \mathrm{H}, J=13.8,2.8 \mathrm{~Hz}$ ), 2.37 (ddd, $1 \mathrm{H}, J=14.2,10.2,5.4 \mathrm{~Hz}$ ), 3.44 (dd, $1 \mathrm{H}, J=10.3,2.2 \mathrm{~Hz}$ ), 3.71 (dd, $1 \mathrm{H}, J=10.3,2.2 \mathrm{~Hz}$ ), 3.80 (d, $1 \mathrm{H}, J=10.8 \mathrm{~Hz}$ ), 4.04-4.08 (m, $1 \mathrm{H}), 4.14$ (dm, $1 \mathrm{H}, J=6.8$ Hz), $4.26(\mathrm{dm}, 1 \mathrm{H}, J=10.0 \mathrm{~Hz}), 4.55(\mathrm{~d}, 1 \mathrm{H}, J=11.9 \mathrm{~Hz}), 4.67$ (d, $1 \mathrm{H}, J=11.9 \mathrm{~Hz}), 5.29$ (ddd, $1 \mathrm{H}$, $J=10.4,1.8,0.9 \mathrm{~Hz}$ ), 5.36 (ddd, $1 \mathrm{H}, J=17.4,1.8,1.1 \mathrm{~Hz}$ ), 6.01 (ddd, $1 \mathrm{H}, J=17.2,10.5,6.9 \mathrm{~Hz}$ ), 7.23-7.32 (m, 5 H). ${ }^{13} \mathbf{C}$ NMR (75 MHz) $\delta \quad 37.4,71.9,73.2,73.7,77.2,85.6,118.3,127.9$ (2 C), 128.0, 128.6 (2 C), 134.0. IR (film): 3434, 2960, 2855, 1737, 1454, 1414, 1260, 1090, 865, 803, 753, $698 \mathrm{~cm}^{-1}$. MS (ES): $257[\mathrm{M}+\mathrm{Na}]^{+}(100 \%), 235[\mathrm{M}+\mathrm{H}]^{+}$. Anal. calcd for $\mathrm{C}_{14} \mathrm{H}_{18} \mathrm{O}_{3}: \mathrm{C}, 71.77 ; \mathrm{H}, 7.74$.

Found: C, 72.01; H, 8.02.

\subsection{Synthesis of (+)-(2S,4S,5S)-2-(tert-Butyldimethylsilyloxymethyl)-5-vinyl-tetrahydrofuran-4-} ol, (+)-26a, and (-)-(2S,4R,5S)-2-(tert-Butyldimethylsilyloxymethyl)-5-vinyl-tetrahydrofuran-4-ol, $(-)-32 a$.

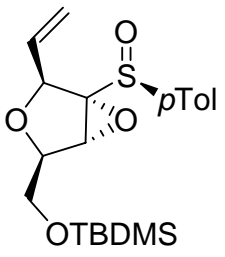

$(+)-28 a$

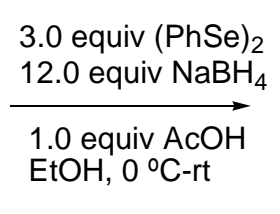

1.0 equiv $\mathrm{AcOH}$

EtOH, O

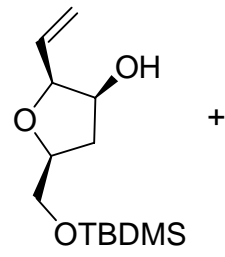

$(+)-26 a(83)$

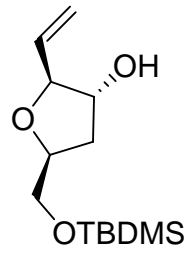

$(-)-32 a(17)$

From a solution of epoxy sulfoxide (+)-28a $(250 \mathrm{mg}, 0.634 \mathrm{mmol}, 1.0$ equiv) in $2.1 \mathrm{~mL}$ of EtOH and a suspension generated from $(\mathrm{PhSe})_{2}\left(593.7 \mathrm{mg}, 1.902 \mathrm{mmol}, 3.0\right.$ equiv) in $9.5 \mathrm{~mL}$ of EtOH and $\mathrm{NaBH}_{4}$ (287.8 mg, $7.61 \mathrm{mmol}, 12.0$ equiv), with $\mathrm{AcOH}$ (36.3 $\mu \mathrm{L}, 0.634 \mathrm{mmol}, 1.0$ equiv) according to the general procedure $(1 \mathrm{~h} 30 \mathrm{~min})$, an 83:17 mixture of alcohols $(+)-26 \mathbf{a}$ and $(-)-32 \mathbf{a}$ was obtained. Purification by chromatography (5-20\% EtOAc-hexane) gave (+)-26a (110 mg, 67\%) and (-)-32a (22 $\mathrm{mg}, 13 \%$ ) as colorless oils.

Data for (+)-26a: $\quad \boldsymbol{R}_{\boldsymbol{f}}=0.22\left(20 \%\right.$ EtOAc-hexane). $[\alpha]^{\mathbf{2 0}}{ }_{\mathbf{D}}=+41.2(c=2.30) .{ }^{\mathbf{1}} \mathbf{H} \mathbf{N M R}(\mathbf{3 0 0} \mathbf{~ M H z})$ $\delta 0.10$ (s, $6 \mathrm{H}), 0.91$ (s, $9 \mathrm{H}), 1.96$ (dd, $1 \mathrm{H}, J=13.8,2.8 \mathrm{~Hz}), 2.36$ (ddd, $1 \mathrm{H}, J=13.9,10.3,4.9 \mathrm{~Hz}$ ), $3.53(\mathrm{dd}, 1 \mathrm{H}, J=10.8,1.8 \mathrm{~Hz}), 3.87$ (dd, $1 \mathrm{H}, J=10.7,2.2 \mathrm{~Hz}), 4.02(\mathrm{~m}, 1 \mathrm{H}), 4.12(\mathrm{dm}, 1 \mathrm{H}), 4.22$ (dq, $1 \mathrm{H}, J=10.1,2.2 \mathrm{~Hz}$ ), 5.25 (ddd, $1 \mathrm{H}, J=10.5,1.9,1.0 \mathrm{~Hz}$ ), 5.34 (dt, $1 \mathrm{H}, J=17.3,1.5 \mathrm{~Hz}$ ), 5.99 (ddd, $1 \mathrm{H}, J=17.3,10.5,6.8 \mathrm{~Hz}) .{ }^{13} \mathrm{C}$ NMR (75 MHz) $\delta-5.7,-5.5,18.5,25.9,37.1,65.5,73.1,77.6$, 85.5, 117.9, 134.1. IR (film): 3436, 3079, 2956, 2930, 2884, 2858, 1725, 1645, 1472, 1255, 1185, 1066, 918, 836, 778, $665 \mathrm{~cm}^{-1}$. MS (EI): $281[\mathrm{M}+\mathrm{Na}]^{+}, 259[\mathrm{M}+\mathrm{H}]^{+}(100 \%)$. Anal. calcd for $\mathrm{C}_{13} \mathrm{H}_{26} \mathrm{O}_{3} \mathrm{Si}: \mathrm{C}, 60.42 ; \mathrm{H}, 10.14$. Found: $\mathrm{C}, 60.73 ; \mathrm{H}, 10.33$.

Data for (-)-32a: $\boldsymbol{R}_{\boldsymbol{f}}=0.22\left(30 \%\right.$ EtOAc-hexane) $[\alpha]^{20}{ }_{\mathbf{D}}=-22.1(c=2.0) .{ }^{1} \mathbf{H}$ NMR (300 MHz) $\delta$ 0.041 (s, 3 H), 0.043 (s, 3 H), 0.09 (s, 9 H), 1.88 (ddd, 1 H, $J=12.9,6.8,3.7$ Hz), 2.04 (ddd, 1 H, $J=$ 
12.9, 8.1, 6.1 Hz), $3.64(\mathrm{dd}, 1 \mathrm{H}, J=10.9,4.4 \mathrm{~Hz}), 3.68(\mathrm{dd}, 1 \mathrm{H}, J=10.7,4.1 \mathrm{~Hz}), 4.11-4.18(\mathrm{~m}, 2 \mathrm{H})$, 4.24 (ddd, $1 \mathrm{H}, J=8.2,6.6,4.2 \mathrm{~Hz}), 5.13(\mathrm{ddd}, 1 \mathrm{H}, J=9.8,1.7,1.2 \mathrm{~Hz}), 5.31$ (ddd, $1 \mathrm{H}, J=17.1,1.9$, $0.7 \mathrm{~Hz}), 5.81(\mathrm{ddd}, 1 \mathrm{H}, J=17.3,10.5,6.3 \mathrm{~Hz}) .{ }^{13} \mathrm{C}$ NMR (75 MHz) $\delta-5.4,-5.3,18.3,25.9,36.1$, 65.3, 76.5, 78.7, 87.4, 116.7, 137.1. IR (film): 3413, 3079, 2949, 2929, 2891, 2858, 1725, 1642, 1472, 1255, 1098, 924, 837, 777, $\mathrm{cm}^{-1}$. MS (EI): $281[\mathrm{M}+\mathrm{Na}]^{+}, 259[\mathrm{M}+\mathrm{H}]^{+}(100 \%)$. Anal. calcd for $\mathrm{C}_{13} \mathrm{H}_{26} \mathrm{O}_{3} \mathrm{Si}: \mathrm{C}, 60.42 ; \mathrm{H}, 10.14$. Found: $\mathrm{C}, 60.20 ; \mathrm{H}, 10.40$.

\subsection{Desilylation of $(+)-26 a$.}

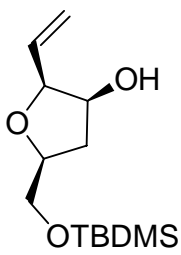

(+)-26a

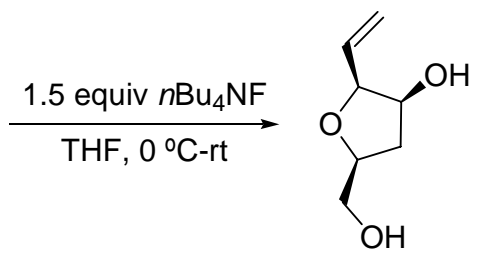

(+)-26d

From (+)-26a (61 mg, $0.237 \mathrm{mmol}$, 1.0 equiv), with $n \mathrm{Bu}_{4} \mathrm{NF}$ (113.6 mg, 0.36, 1.5 equiv), following the general procedure (1 h $30 \mathrm{~min}$ ) and after chromatography (20-30\% EtOAc-hexane), diol (+)-26d (34 $\mathrm{mg}, 100 \%$ ) was obtained. This product had identical spectral data to that found before.

\subsection{General Procedure for the Oxidation of Hydroxy Vinyl Tetrahydrofurans to Dioxabicyclic}

\section{Lactols.}

To a solution of the substrate in 4:1 DMF: $\mathrm{H}_{2} \mathrm{O}(10 \mathrm{~mL} / \mathrm{mmol})$ was added at $\mathrm{rt}^{\mathrm{PdCl}_{2}}(0.5$ equiv $)$ and $\mathrm{CuCl}$ (6.0 equiv) or $\mathrm{Cu}(\mathrm{OAc})_{2}$ (1.0-1.5 equiv) and oxygen was bubbled slowly through the mixture with stirring. Upon completion (TLC), the mixture was diluted with EtOAc $(10 \mathrm{~mL} / \mathrm{mmol})$, filtered through a short pad of silica gel or celite with EtOAc as eluent. The resulting solution was washed with $\mathrm{H}_{2} \mathrm{O}(2$ $\mathrm{mL} / \mathrm{mmol}$ ), dried over $\mathrm{MgSO}_{4}$, filtered and concentrated under reduced pressure to give a crude product that was purified by chromatography on silica gel using the appropriate mixture of eluents.

\subsection{Synthesis of ent-Kumausyne precursor, (-)-(4S,6S,8R)-6-(tert-Butyldiphenylsilyloxymethyl)-} 1,5-dioxabicyclo[3.3.0 $\left.{ }^{4,8}\right]$ octan-2-ol, (-)-25b. ${ }^{3}$

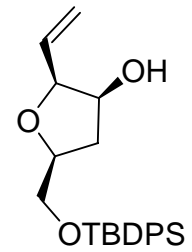

(+)-26b

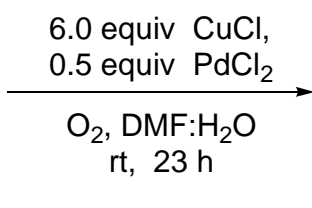

$\mathrm{t}, 23 \mathrm{~h}$

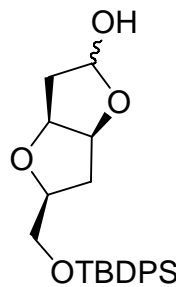

(-)-25b 
From alcohol (+)-26b (18.4 mg, $0.048 \mathrm{mmol}, 1.0$ equiv) in $0.5 \mathrm{~mL}$ of aqueous DMF, with $35 \mathrm{mg}$ of a mixture of $\mathrm{PdCl}_{2}$ and $\mathrm{CuCl}\left(0.5\right.$ equiv $\mathrm{PdCl}_{2}$ and 6.0 equiv $\left.\mathrm{CuCl}\right)$, following the general procedure $(23$ h), lactol (-)-25b (13.14 mg, 69\%) was obtained as a colorless oil (74:26 mixture of anomers) after chromatography (10-50\% EtOAc-hexane).

Data for (-)-25b (4: 26 mixture of anomers): $\boldsymbol{R}_{\boldsymbol{f}}=0.26\left(30 \%\right.$ EtOAc-hexane). $[\alpha]^{\mathbf{2 0}}{ }_{\mathbf{D}}=-16.0(c=$ 0.46). ${ }^{1}$ H NMR (300 MHz) $\delta 1.06$ and 1.08 (2 s, $\left.9 \mathrm{H}\right), 2.03(\mathrm{dt}, 2 \mathrm{H}, J=14.0,4.8 \mathrm{~Hz}), 2.26-2.31$ (m, $2 \mathrm{H}), \underline{2.36}(\mathrm{~s}, 1 \mathrm{H}), \underline{3.57}(\mathrm{dd}, 1 \mathrm{H}, J=11.4,3.5 \mathrm{~Hz}), 3.64(\mathrm{dd}, 1 \mathrm{H}, J=10.4,5.4 \mathrm{~Hz}), 3.71(\mathrm{dd}, 1 \mathrm{H}, J=$ 10.4, $5.7 \mathrm{~Hz}), \underline{3.87}(\mathrm{dd}, 1 \mathrm{H}, J=11.3,3.2 \mathrm{~Hz}), \underline{3.97}(\mathrm{~m}, 1 \mathrm{H}), 4.11(\mathrm{~m}, 1 \mathrm{H}), \underline{4.48}(\mathrm{t}, 1 \mathrm{H}, J=4.3 \mathrm{~Hz})$, $4.58(\mathrm{~m}, 1 \mathrm{H}), \underline{4.72}(\mathrm{q}, 1 \mathrm{H}, J=4.1 \mathrm{~Hz}), 4.79(\mathrm{~m}, 1 \mathrm{H}, \mathrm{H}-4), \underline{5.38}(\mathrm{dd}, 1 \mathrm{H}, J=12.1,4.9 \mathrm{~Hz}), 5.54$ (m, 1 H), 7.33-7.44 (m, $6 \mathrm{H})$ 7.63-7.68 (m, 4 H). ${ }^{13}$ C NMR (50 MHz) $\delta \underline{19.2}, 19.3,26.8, \underline{26.8}(3 \mathrm{C}), \underline{35.1}$, $36.8, \underline{41.5}, 41.7, \underline{64.2}, 66.4, \underline{81.1}, 81.5, \underline{83.2}, 83.6, \underline{84.8}, 84.8, \underline{100.2}, 100.2, \underline{127.6}, 127.7, \underline{129.6}, 129.7(2$ C), $\underline{133.1}, 133.2, \underline{135.6}, 137.5$ (2 C). IR (film): 3400, 3060, 3040, 2950, 2920, 1465, 1420, $1110 \mathrm{~cm}^{-1}$. Anal. calcd for $\mathrm{C}_{23} \mathrm{H}_{30} \mathrm{O}_{4} \mathrm{Si}: \mathrm{C}, 69.31 ; \mathrm{H}, 7.59$. Found: $\mathrm{C}, 69.66 ; \mathrm{H}, 7.82$.

9.3. Wacker oxidation of diol (+)-26d. Synthesis of (-)-(4S,6S,8R)-6-(hydroxymethyl)-1,5dioxabicyclo[3.3.0 $\left.{ }^{4,8}\right]$ octan-2-ol, (-)-25d.

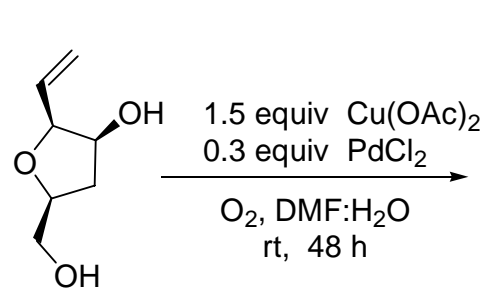

$(+)-26 d$

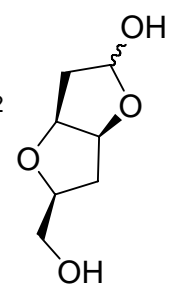

$(-)-25 d$

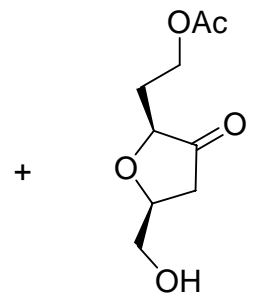

$(-)-33$

From alcohol (+)-26d (34 mg, $0.237 \mathrm{mmol}, 1.0$ equiv) in $2.4 \mathrm{~mL}$ of aqueous $\mathrm{DMF}, \mathrm{Cu}(\mathrm{OAc})_{2}(47.3$ $\mathrm{mg}, 0.237 \mathrm{mmol}, 1.0$ equiv) and $\mathrm{PdCl}_{2}(8.3 \mathrm{mg}, 0.047 \mathrm{mmol}, 0.2$ equiv), with a second addition after 40 $\mathrm{h}$ of $\mathrm{Cu}(\mathrm{OAc})_{2}\left(23.6 \mathrm{mg}, 0.5\right.$ equiv) and $\mathrm{PdCl}_{2}$ ( $4.1 \mathrm{mg}, 0.1$ equiv), following the general procedure (48 h), and after chromatography (5\%-10\% EtOH- $\left.\mathrm{CH}_{2} \mathrm{Cl}_{2}\right)$, lactol (-)-25d (20 mg, 53\%) along with ketone (-)-33 (10 mg, 21\%), were obtained as colorless oils.

Data for (-)-25d: $\quad \boldsymbol{R}_{\boldsymbol{f}}=0.20\left(10 \% \mathrm{EtOH}-\mathrm{CH}_{2} \mathrm{Cl}_{2}\right) .[\alpha]^{20}{ }_{\mathbf{D}}=-34.9(c=1.0) .{ }^{1} \mathbf{H} \mathbf{N M R}(300 \mathrm{MHz}) \delta$ 2.16-2.31 (m, $2 \mathrm{H}), 2.44$ (dd, $1 \mathrm{H}, J=18.2,6.5 \mathrm{~Hz}), 2.63$ (dd, $1 \mathrm{H}, J=18.3,9.8 \mathrm{~Hz}), 2.90$ (br s, $1 \mathrm{H}$ ), 3.40 (br s, $1 \mathrm{H}), 3.56-3.80(\mathrm{~m}, 2 \mathrm{H}), 3.90(\mathrm{~m}, 1 \mathrm{H}), 4.58(\mathrm{~m}, 1 \mathrm{H}), 4.72(\mathrm{~m}, 1 \mathrm{H}), 5.45(\mathrm{~d}, 1 \mathrm{H}, J=4.9$ $\mathrm{Hz}), 5.68(\mathrm{~m}, 1 \mathrm{H}) .{ }^{13} \mathrm{C}$ NMR (75 MHz) $\delta$ 35.6, $\underline{37.3}, 39.7, \underline{40.4}, 62.4, \underline{63.1}, \underline{81.0}, 81.2, \underline{83.6}, 83.9$, $\underline{86.0}, 86.5, \underline{99.9}, 100.0$. IR (film): 3400, 2913, 2847, 1754, 1663, 1436, 1241, 1067, $753 \mathrm{~cm}^{-1}$. MS (ES): $183[\mathrm{M}+\mathrm{Na}]^{+}(100 \%), 161[\mathrm{M}+\mathrm{H}]^{+}$. Anal. calcd for $\mathrm{C}_{7} \mathrm{H}_{12} \mathrm{O}_{4}: \mathrm{C}, 52.49 ; \mathrm{H}, 7.55$. Found: $\mathrm{C}$, 52.23; H, 7.24. 
Data for (-)-33: $\boldsymbol{R}_{\boldsymbol{f}}=0.15\left(25 \%\right.$ EtOAc- $\left.\mathrm{CH}_{2} \mathrm{Cl}_{2}\right) .[\alpha]^{\mathbf{2 0}}{ }_{\mathbf{D}}=-38.9(c=0.80) .{ }^{1} \mathbf{H} \mathbf{N M R}(300 \mathrm{MHz}) \delta$ 1.91-1.98 (m, $1 \mathrm{H}), 2.00$ (s, $3 \mathrm{H}), 2.02-2.15$ (m, $1 \mathrm{H}), 2.44$ (dd, $1 \mathrm{H}, J=18.3,6.3 \mathrm{~Hz}), 2.55$ (dd, $1 \mathrm{H}, J=$ 18.3, 9.8 Hz), $3.66(\mathrm{ddd}, 1 \mathrm{H}, J=12.0,7.8,4.2 \mathrm{~Hz}), 3.91$ (dd, $1 \mathrm{H}, J=6.8,4.6 \mathrm{~Hz}), 3.95$ (m, $1 \mathrm{H}), 4.14-$ 4.34 (m, 4 H). ${ }^{13}$ C NMR (75 MHz) $\delta$ 20.8, 29.9, 60.1, 63.4, 76.1, 78.4, 170.8, 214.5. IR (film): 3474, 2926, 1757, 1736, 1389, 1368, 1244, 1172, 1100, 1047, $844 \mathrm{~cm}^{-1}$. MS (ES): $225[\mathrm{M}+\mathrm{Na}]^{+}$. Anal. calcd for $\mathrm{C}_{9} \mathrm{H}_{14} \mathrm{O}_{5}: \mathrm{C}, 53.46 ; \mathrm{H}, 6.98$. Found: $\mathrm{C}, 53.77 ; \mathrm{H}, 7.03$.

\subsection{Wittig reaction on lactol (-)-25d. Synthesis of $(+)-(2 S, 4 S, 5 S)-2-H y d r o x y m e t h y l-5-(p e n t-2-$} en-4-ynyl)-tetrahydrofuran-4-ol. (+)-34.

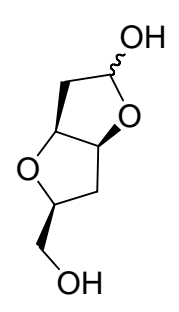

$(-)-25 d$
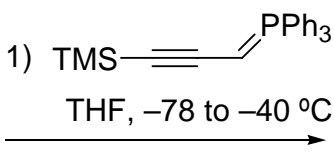

2) $n \mathrm{Bu}_{4} \mathrm{NF}, 0^{\circ} \mathrm{C}-\mathrm{rt}$

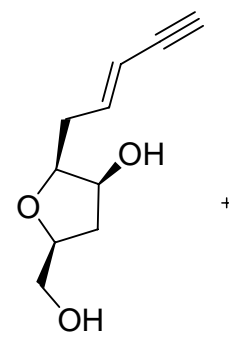

$(+)-34-E(86)$

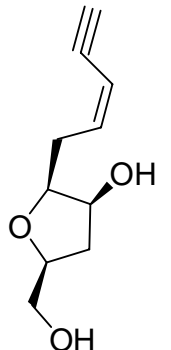

$(+)-34-Z(14)$

To a cold $\left(-78^{\circ} \mathrm{C}\right)$ solution of (3-trimethylsilyl-2-propynyl) triphenylphosphonium bromide (541.4 $\mathrm{mg}, 1.194 \mathrm{mmol}, 6.0$ equiv) in THF $(6.0 \mathrm{~mL})$ was added dropwise $n$-BuLi $(0.74 \mathrm{~mL}, 1.194 \mathrm{mmol}, 6.0$ equiv, 1.6 $\mathrm{M}$ in hexane). The mixture was allowed to warm up to $-40{ }^{\circ} \mathrm{C}$, stirred for 30 min and then cooled back to $-78{ }^{\circ} \mathrm{C}$. Then, $3 \mathrm{~mL}$ of this phosphorane solution (ca. 3 equiv) was added dropwise to a cold $\left(-78^{\circ} \mathrm{C}\right)$ solution of lactol (-)-25d (32 $\mathrm{mg}, 0.199 \mathrm{mmol}, 1.0$ equiv) in THF $(2 \mathrm{~mL})$. The bath was then replaced for a $0{ }^{\circ} \mathrm{C}$ bath and the mixture was allowed to warm up slowly to rt. After $15 \mathrm{~h}$, TLC analysis revealed disappearance of lactol (-)-25d. The mixture was cooled again to $0{ }^{\circ} \mathrm{C}, n \mathrm{Bu}_{4} \mathrm{NF}(251.2$ mg, $0.796 \mathrm{mmol}, 4.0$ equiv) was added and the mixture was allowed to warm up to rt. After $4 \mathrm{~h}$ and 30 min, the reaction was quenched with saturated $\mathrm{NH}_{4} \mathrm{Cl}$ solution $(10 \mathrm{~mL} / \mathrm{mmol})$, the mixture was concentrated to dryness and the residue was purified by chromatography on silica gel $(15 \%-50 \%$ EtOAc- $\left.\mathrm{CH}_{2} \mathrm{Cl}_{2}\right)$ to yield (+)-34 (30 $\left.\mathrm{mg}, 84 \%\right)$ as an inseparable 86:14 E/Z mixture.

Data for (+)-34-E (from the mixture): $\boldsymbol{R}_{\boldsymbol{f}}=0.20\left(5 \%\right.$ EtOH $\left.-\mathrm{CH}_{2} \mathrm{Cl}_{2}\right) . \quad[\alpha]^{\mathbf{2 0}}=+26.7(c=0.6) .{ }^{\mathbf{1}} \mathbf{H}$ NMR (300 MHz) $\delta 1.89(\mathrm{dd}, 1 \mathrm{H}, J=14.2,3.2 \mathrm{~Hz}), 2.36(\mathrm{ddd}, 1 \mathrm{H}, J=14.2,10.3,5.4 \mathrm{~Hz}), 2.49$ (t, 2 $\mathrm{H}, J=7.1 \mathrm{~Hz}), 2.78(\mathrm{~d}, 1 \mathrm{H}, J=2.2 \mathrm{~Hz}), 3.51(\mathrm{dd}, 1 \mathrm{H}, J=11.7,2.2 \mathrm{~Hz}), 3.68(\mathrm{dt}, 1 \mathrm{H}, J=6.8,2.7 \mathrm{~Hz})$, $3.85(\mathrm{dd}, 1 \mathrm{H}, J=11.7,2.2 \mathrm{~Hz}), 4.04(\mathrm{dd}, 1 \mathrm{H}, J=5.4,2.7 \mathrm{~Hz}), 4.17(\mathrm{qd}, 1 \mathrm{H}, J=10.2,2.3 \mathrm{~Hz}), 5.58$ $(\mathrm{ddt}, 1 \mathrm{H}, J=16.1,2.2,1.5 \mathrm{~Hz}), 6.27(\mathrm{dt}, 1 \mathrm{H}, J=15.9,7.3 \mathrm{~Hz}) .{ }^{13} \mathbf{C} \mathbf{N M R}(75 \mathrm{MHz}) \delta 32.8,37.2$, 64.1, 71.5, 76.9, 77.3, 82.4, 83.2, 111.0, 142.4. IR (film): 3430, 3231, 2920, 2876, 1627, 1443, 1107, 
1082, 1035, $966 \mathrm{~cm}^{-1}$. MS (ES): $365[2 \mathrm{M}+\mathrm{H}]^{+}, 205[\mathrm{M}+\mathrm{Na}]^{+}, 183[\mathrm{M}+\mathrm{H}]^{+}$. Anal. calcd for $\mathrm{C}_{10} \mathrm{H}_{14} \mathrm{O}_{3}$ : C, 65.91; H, 7.74. Found: C, 66.22; H, 7.59.

Partial data for $(+)-\mathbf{3 4 - Z}$ (from the mixture): ${ }^{\mathbf{1}} \mathbf{H}$ NMR (300 MHz) $\delta 2.71(\mathrm{~m}, 1 \mathrm{H}), 3.74(\mathrm{dt}, 1 \mathrm{H})$, $5.50(\mathrm{dm}, 1 \mathrm{H}), 6.11(\mathrm{dm}, 1 \mathrm{H})$.

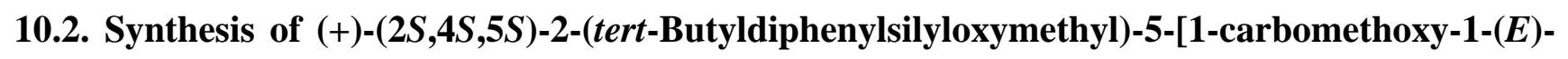
propen-3-yl]tetrahydrofuran-4-ol, (+)-35b.

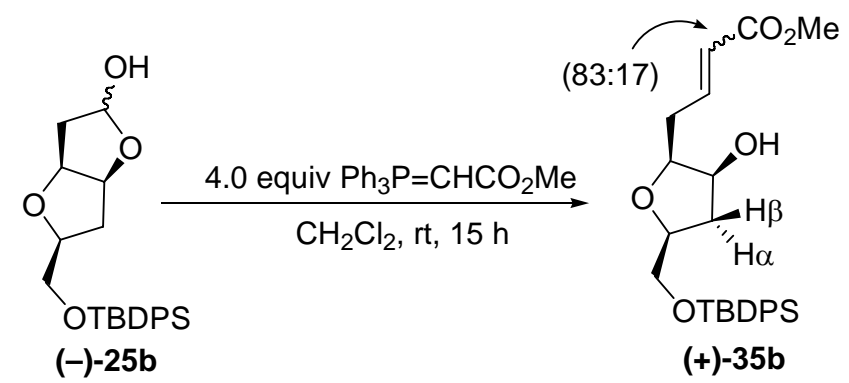

To a solution of lactol (-)-25b (13.10 mg, $0.033 \mathrm{mmol}, 1.0$ equiv) in $0.7 \mathrm{~mL}$ of $\mathrm{CH}_{2} \mathrm{Cl}_{2}$ was added at rt methyl (triphenylphosphoranylidene)acetate ( $44 \mathrm{mg}, 0.132 \mathrm{mmol}, 4.0$ equiv) and the reaction mixture was stirred at $\mathrm{rt}$ for $15 \mathrm{~h}$. The solvent was then removed and the residue was purified by chromatography (10-50\% EtOAc-hexane) to give alkenes 35b (12.6 mg, 85\%, 83:17 E: $Z$ mixture) as a colorless oil. Pure $E$ isomer (+)-35b was isolated after a second, more careful, chromatography.

Data for (+)-35b: $\quad \boldsymbol{R}_{\boldsymbol{f}}=0.30\left(30 \%\right.$ EtOAc-hexane). $[\alpha]^{\mathbf{2 0}}{ }_{\mathbf{D}}=+22.8(c=0.49) .{ }^{\mathbf{1}} \mathbf{H} \mathbf{~ N M R}(\mathbf{2 0 0} \mathbf{~ M H z})$ $\delta 1.06(\mathrm{~s}, 9 \mathrm{H}), 1.24$ (br s, $1 \mathrm{H}), 2.06$ (dd, $1 \mathrm{H}, J=14.0,2.9 \mathrm{~Hz}), 2.37$ (ddd, $1 \mathrm{H}, J=14.4,10.5,4.2 \mathrm{~Hz}$ ), $2.65(\mathrm{~m}, 2 \mathrm{H}), 3.44(\mathrm{dm}, 1 \mathrm{H}, J=11.1 \mathrm{~Hz}), 3.72(\mathrm{~s}, 3 \mathrm{H}), 3.75(\mathrm{dm}, 1 \mathrm{H}, J=6.7 \mathrm{~Hz}), 3.84(\mathrm{dd}, 1 \mathrm{H}, J=$ 11.2, $2.2 \mathrm{~Hz}), 4.01(\mathrm{~m}, 1 \mathrm{H}), 4.15(\mathrm{dm}, 1 \mathrm{H}, J=11.0 \mathrm{~Hz}), 5.98$ (dt, $1 \mathrm{H}, J=15.7,1.5 \mathrm{~Hz}), 7.07$ (dt, $1 \mathrm{H}$, $J=15.7,7.3 \mathrm{~Hz}), 7.34-7.45(\mathrm{~m}, 6 \mathrm{H}), 7.65-7.75(\mathrm{~m}, 4 \mathrm{H}) .{ }^{13} \mathbf{C}$ NMR (50 MHz) $\delta \quad 19.1,26.8(3 \mathrm{C})$, 32.5, 37.1, 51.3, 66.1, 71.8, 77.5, 82.8, 122.9, 127.8 (2 C), 127.9 (2 C), 129.1, 130.0, 132.0, 132.4, 135.6 (2 C), 135.7 (2 C), 146.0, 166.9. IR $\left(\mathrm{CCl}_{4}\right): 3440,3060,2940,1720,1660,1430,1320,1260$, $1100,1040,800,700 \mathrm{~cm}^{-1}$. Anal. calcd for $\mathrm{C}_{26} \mathrm{H}_{34} \mathrm{O}_{5} \mathrm{Si}: \mathrm{C}, 68.69 ; \mathrm{H}, 7.54$. Found: $\mathrm{C}, 68.93 ; \mathrm{H}, 7.25$.

Partial data for 35b-Z (minor, from an 83:17 mixture): $\boldsymbol{R}_{\boldsymbol{f}}=0.32$ (30\% EtOAc-hexane). ${ }^{\mathbf{1}} \mathbf{H}$ NMR (300 MHz) $\delta 2.05(\mathrm{dd}, 1 \mathrm{H}, J=13.7,2.7 \mathrm{~Hz}), 3.09$ (t, $1 \mathrm{H}, J=7.2 \mathrm{~Hz}), 3.68(\mathrm{~s}, 3 \mathrm{H}), 4.07$ (dd, $1 \mathrm{H}, J=$ 4.6, $1.9 \mathrm{~Hz}), 5.88(\mathrm{dm}, 1 \mathrm{H}, J=11.5 \mathrm{~Hz}), 6.44(\mathrm{dt}, 1 \mathrm{H}, J=11.6,7.6 \mathrm{~Hz})$. 
10.3. Synthesis of $\quad(2 S, 4 S, 5 S)-5-[1-\operatorname{carbomethoxy-1-(E)-propen-3-yl]-2-}$ hydroxymethyltetrahydrofuran-4-ol, 35d.

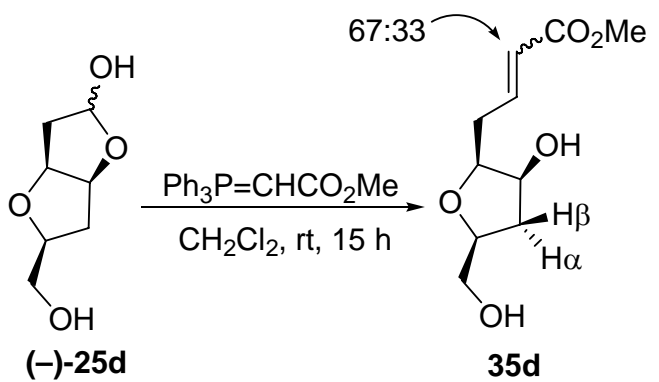

To a solution of lactol (-)-25d (3 mg, 1.0 equiv) in $0.5 \mathrm{~mL}$ of $\mathrm{CH}_{2} \mathrm{Cl}_{2}$ was added at $\mathrm{rt}$ methyl (triphenylphosphoranylidene)acetate (33 mg, ca. 3.0 equiv) and the reaction mixture was stirred at $\mathrm{rt}$ for $15 \mathrm{~h}$. The solvent was then removed and the residue was purified by chromatography (10-100\% EtOAchexane) to give alkenes 35d as a 67:33 $E / Z$ mixture. The yield was not determined.

Data for $E$ isomer 35d: $\boldsymbol{R}_{\boldsymbol{f}}=0.33$ (EtOAc). ${ }^{1} \mathbf{H}$ NMR (300 MHz) $\delta 1.91(\mathrm{dd}, 1 \mathrm{H}, J=16.8,2.8 \mathrm{~Hz})$, 2.37 (ddd, $1 \mathrm{H}, J=16.8,9.8,5.6 \mathrm{~Hz}), 2.57$ (t, $2 \mathrm{H}, J=7.0 \mathrm{~Hz}), 3.49$ (m, $2 \mathrm{H}), 3.70$ (s, $3 \mathrm{H}), 3.74$ (td, 1 $\mathrm{H}, J=7.5,2.5 \mathrm{~Hz}), 3.86(\mathrm{~m}, 1 \mathrm{H}), 4.40(\mathrm{~m}, 1 \mathrm{H}), 4.58(\mathrm{~m}, 1 \mathrm{H}), 5.94(\mathrm{dt}, 1 \mathrm{H}, J=15.8,1.7 \mathrm{~Hz}), 7.00$ (dt, $1 \mathrm{H}, J=15.8,7.9 \mathrm{~Hz})$.

\subsection{Synthesis of ent-Kumausallene precursor, (-)-(2R,4S,6S,8R)-2-Carbomethoxymethyl-1,5-} dioxabicyclo[3.3.0 $\left.{ }^{4,8}\right]$ octan-6-methanol, (-)-27. ${ }^{4}$

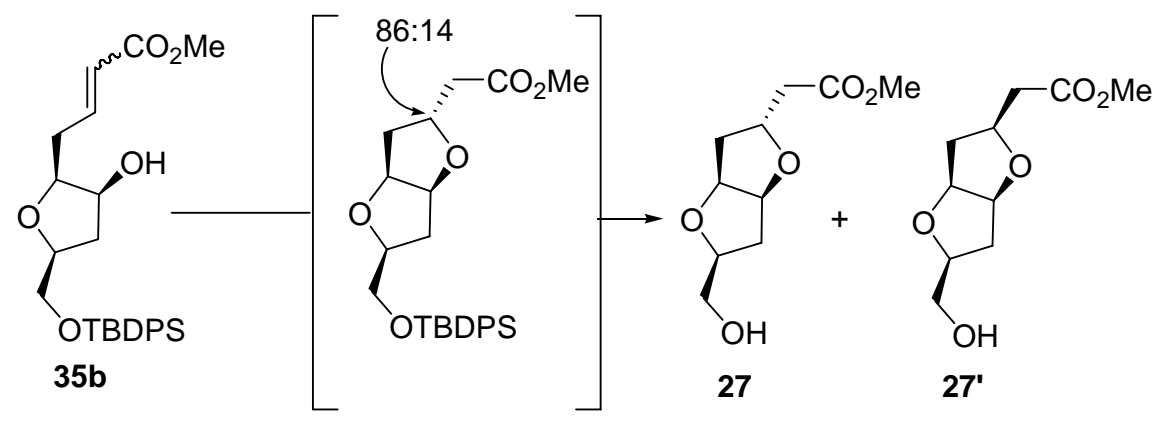
1) $\mathrm{NaOMe}, \mathrm{MeOH}, \mathrm{rt}, 19 \mathrm{~h}$
2) 1.5 equiv $n \mathrm{Bu}_{4} \mathrm{NF}$,
2.0 equiv $\mathrm{AcOH}$
86
14
THF, rt, $22 \mathrm{~h}$
1) 2.5 equiv $n \mathrm{Bu}_{4} \mathrm{NF}$
THF, rt, $36 \mathrm{~h}$
2) $\mathrm{NaOMe}, \mathrm{MeOH}, \mathrm{rt}, 22 \mathrm{~h}$

$\begin{array}{ll}72 & 28 \\ 75 & 25\end{array}$

To a solution of the 83:17 E,Z mixture of unsaturated esters (+)-35b (12.6 mg, $0.028 \mathrm{mmol}, 1.0$ equiv) in $0.1 \mathrm{~mL}$ of $\mathrm{MeOH}$ was added at $\mathrm{rt} 0.2 \mathrm{~mL}$ of a solution of $\mathrm{NaOMe}$ in $\mathrm{MeOH}$ (freshly prepared from a catalytic amount of $\mathrm{Na}$ and $\mathrm{MeOH}$ ). The mixture was stirred at $\mathrm{rt}$ for $19 \mathrm{~h}$ and then it was quenched with $1 \mathrm{~mL}$ of $\mathrm{H}_{2} \mathrm{O}$, diluted with $10 \mathrm{~mL}$ of EtOAc and the layers were separated. The aqueous layer was 
extracted with $2 \mathrm{~mL}$ of EtOAc and the combined organic extracts were washed with $2 \mathrm{~mL}$ of a saturated solution of $\mathrm{NaCl}$, dried over $\mathrm{MgSO}_{4}$ and concentrated to give an inseparable 86:14 mixture of bicyclic products, epimeric at C-2. This crude was dissolved in $0.4 \mathrm{~mL}$ of THF and $\mathrm{AcOH}$ ( $c$. $3 \mu \mathrm{L}, 0.056$ mmol, 2.0 equiv), followed by $n \mathrm{Bu}_{4} \mathrm{NF}$ ( $42 \mu \mathrm{L}, 1 \mathrm{M}$ in THF, $0.042 \mathrm{mmol}, 1.5$ equiv) was added. The mixture was stirred for $22 \mathrm{~h}$, the solvent was removed and the residue was purified by chromatography (30-100\% EtOAc-hexane) to give an inseparable 86:14 mixture of (-)-27 and 27' (3.67 $\mathrm{mg}, 64 \%$ ) as a colorless oil.

In a related experiment, to an $83: 17$ mixture of (+)-35b (10.6 mg, $0.023 \mathrm{mmol}, 1.0$ equiv) in $0.5 \mathrm{~mL}$ of THF was added $n \mathrm{Bu}_{4} \mathrm{NF}(0.23 \mathrm{~mL}, 2.5 \mathrm{M}$ en THF, $0.058 \mathrm{mmol}, 2.5$ equiv) and the mixture was stirred at $\mathrm{rt}$ for $36 \mathrm{~h}$. The solvent was removed and the residue was purified to give an inseparable 72:28 mixture of (-)-27 and 27' $(2.8 \mathrm{mg}, 63 \%)$ as a colorless oil. Treatment of this mixture with catalytic $\mathrm{NaOMe}$ in $\mathrm{MeOH}$ for 2 days rendered a 75:25 mixture of (-)-27 and 27'.

Data for the tert-Butyldiphenylsilyl ether of 27 (major): $\boldsymbol{R}_{\boldsymbol{f}}=0.31$ (30\% EtOAc-hexane). ${ }^{1} \mathbf{H}$ NMR (300 MHz) $\delta 1.04$ (s, 9 H), 1.57 (ddd, $1 \mathrm{H}, J=13.3,10.4,5.0 \mathrm{~Hz}$ ), 1.84 (ddd, $1 \mathrm{H}, J=13.7,9.0,3.7$ Hz), 2.21 (m, 2 H), 2.48 (dd, 1 H, $J=15.1,6.0 \mathrm{~Hz}), 2.57$ (dd, $1 \mathrm{H}, J=15.1,6.8 \mathrm{~Hz}), 3.65$ (s, $3 \mathrm{H}), 3.73$ (d, $2 \mathrm{H}, J=4.9 \mathrm{~Hz}), 3.91(\mathrm{~m}, 1 \mathrm{H}), 4.36(\mathrm{~m}, 1 \mathrm{H}), 4.48(\mathrm{t}, 1 \mathrm{H}, J=4.8 \mathrm{~Hz}), 4.73(\mathrm{~m}, 1 \mathrm{H}), 7.33-7.40(\mathrm{~m}$, $6 \mathrm{H})$, 7.65-7.69 (m, $4 \mathrm{H})$.

Partial data for the tert-Butyldiphenylsilyl ether of 27' (minor): ${ }^{1} \mathbf{H}$ NMR (300 MHz) $\delta 2.63(\mathrm{dd}, 1$ $\mathrm{H}, J=15.1,6.5 \mathrm{~Hz}), 3.63\left(\mathrm{~s}, 3 \mathrm{H}, \mathrm{CO}_{2} \underline{\mathrm{Me}}\right), 4.78(\mathrm{~m}, 1 \mathrm{H})$.

Data for ent-Kumausallene precursor (-)-27 (from an 86:14 mixture of 27:27'): $\boldsymbol{R}_{\boldsymbol{f}}=0.20$ (EtOAc). $[\alpha]^{20}{ }_{\mathbf{D}}=-11.1(c=0.21) .{ }^{1} \mathbf{H}$ NMR $(300 \mathrm{MHz}) \delta 1.62(\mathrm{ddd}, 1 \mathrm{H}, J=13.4,10.2,4.8 \mathrm{~Hz}), 1.77(\mathrm{ddd}, 1$ $\mathrm{H}, J=12.1,8.8,3.4 \mathrm{~Hz}), 1.92(\mathrm{t}, 1 \mathrm{H}, J=6.0 \mathrm{~Hz}), 2.22(\mathrm{dt}, 1 \mathrm{H}, J=13.8,7.1 \mathrm{~Hz}), 2.27$ (dd, $1 \mathrm{H}, J=$ 13.4, $5.0 \mathrm{~Hz}), 2.51(\mathrm{dd}, 1 \mathrm{H}, J=15.2,6.0 \mathrm{~Hz}), 2.60(\mathrm{dd}, 1 \mathrm{H}, J=15.4,7.0 \mathrm{~Hz}), 3.60(\mathrm{~m}, 1 \mathrm{H}), 3.68(\mathrm{~s}, 3$ H), 3.74 (ddd, $1 \mathrm{H}, J=11.7,6.3,3.1 \mathrm{~Hz}), 3.97$ (m, $1 \mathrm{H}), 4.45$ (m, $1 \mathrm{H}), 4.51$ (t, $1 \mathrm{H}, J=4.8 \mathrm{~Hz}), 4.74$ (m, $1 \mathrm{H}) .{ }^{13} \mathbf{C}$ NMR (50 MHz) $\delta 35.3,39.1,39.5,51.8,64.6,74.0,80.3,83.8,84.2,171.3 . \quad$ IR $\left(\mathrm{CCl}_{4}\right)$ : 3450, 2937, 1744, $1444 \mathrm{~cm}^{-1}$. MS (EI): 185, 167, 141 (100\%), 125, 111, 107, 100, 81, 71, 59, 55, 43. Anal. calcd for $\mathrm{C}_{10} \mathrm{H}_{16} \mathrm{O}_{5}$ : C, 55.55; H, 7.46. Found: $\mathrm{C}, 55.22 ; \mathrm{H}, 7.71$.

Partial data for 27' (minor, from a mixture of $\mathbf{2 7}$ and $\mathbf{2 7}$ ', enriched in 27'): $\boldsymbol{R}_{\boldsymbol{f}}=0.20$ (EtOAc). ${ }^{\mathbf{1}} \mathbf{H}$ NMR (300 MHz) $\delta 1.86(\mathrm{ddd}, 1 \mathrm{H}, J=13.9,6.8,1.7 \mathrm{~Hz}), 1.96(\mathrm{ddd}, 1 \mathrm{H}, J=14.0,6.2,1.6 \mathrm{~Hz}), 2.35$ (ddd, $1 \mathrm{H}, J=13.8,7.6,6.1 \mathrm{~Hz}), 2.77$ (dd, $1 \mathrm{H}, J=19.6,7.3 \mathrm{~Hz}), 4.16(\mathrm{~m}, 1 \mathrm{H}), 4.36$ (m, $1 \mathrm{H})$. MS (EI): 186, 167, 153, 135, 125, 107 (100\%), 81, 71, 59, 55, 43. 
12. References and Notes.

1. Watson, S. C.; Eastham, J. F. J. Organomet. Chem. 1967, 9, 165-168. In some cases this procedure was not completely accurate particularly for "old" bottles of reagent. Then, the reagent was titrated by reaction with a slight excess of 3,4-dimethoxybenzaldehyde in THF, standard work-up and measuring accurate integrals in the ${ }^{1} \mathrm{H}$ NMR of the crude reaction mixture.

2. Fernández de la Pradilla, R.; Manzano, P.; Montero, C.; Priego, J.; Martínez-Ripoll, M.; MartínezCruz, L. A. J. Org. Chem. 2003, 68, 7755-7767.

3. Boukouvalas, J.; Fortier, G.; Radu, I.-I. J. Org. Chem. 1998, 63, 916-917.

4. Grese, T.; Hutchinson, K.; Overman, L. E. J. Org. Chem. 1993, 58, 2468-2477. 